\title{
SKY-SCATTERED SOLAR RADIATION BASED PLUME TRANSMISSIVITY MEASUREMENTS
}

By

\section{Chen Yang}

\author{
A thesis submitted to \\ The Faculty of Graduate Studies and Research \\ In partial fulfillment of the degree requirements of \\ Master of Applied Science
}

Ottawa-Carleton Institute for

Mechanical and Aerospace Engineering

Department of Mechanical and Aerospace Engineering

Carleton University

Ottawa, Ontario, Canada

August 2008

(C) Chen Yang 2008 


$\begin{array}{ll}\begin{array}{l}\text { Library and } \\ \text { Archives Canada }\end{array} & \begin{array}{l}\text { Bibliothèque et } \\ \text { Archives Canada }\end{array} \\ \begin{array}{l}\text { Published Heritage } \\ \text { Branch }\end{array} & \begin{array}{l}\text { Direction du } \\ \text { Patrimoine de l'édition }\end{array} \\ \begin{array}{l}\text { 395 Wellington Street } \\ \text { Ottawa ON K1A 0N4 } \\ \text { Canada }\end{array} & \begin{array}{l}\text { 395, rue Wellington } \\ \text { Ottawa ON K1A 0N4 } \\ \text { Canada }\end{array}\end{array}$

Your file Votre référence ISBN: 978-0-494-44064-3 Our file Notre référence ISBN: 978-0-494-44064-3

NOTICE:

The author has granted a nonexclusive license allowing Library and Archives Canada to reproduce, publish, archive, preserve, conserve, communicate to the public by telecommunication or on the Internet, loan, distribute and sell theses worldwide, for commercial or noncommercial purposes, in microform, paper, electronic and/or any other formats.

The author retains copyright ownership and moral rights in this thesis. Neither the thesis nor substantial extracts from it may be printed or otherwise reproduced without the author's permission.
AVIS:

L'auteur a accordé une licence non exclusive permettant à la Bibliothèque et Archives Canada de reproduire, publier, archiver, sauvegarder, conserver, transmettre au public par télécommunication ou par l'Internet, prêter, distribuer et vendre des thèses partout dans le monde, à des fins commerciales ou autres, sur support microforme, papier, électronique et/ou autres formats.

L'auteur conserve la propriété du droit d'auteur et des droits moraux qui protège cette thèse. $\mathrm{Ni}$ la thèse ni des extraits substantiels de celle-ci ne doivent être imprimés ou autrement reproduits sans son autorisation.
In compliance with the Canadian Privacy Act some supporting forms may have been removed from this thesis.

While these forms may be included in the document page count, their removal does not represent any loss of content from the thesis.
Conformément à la loi canadienne sur la protection de la vie privée, quelques formulaires secondaires ont été enlevés de cette thèse.

Bien que ces formulaires aient inclus dans la pagination, il n'y aura aucun contenu manquant.

\section{Canada}




\section{Abstract}

A new sky-scattered solar radiation based line-of-sight optical attenuation technique (skyLOSA) has been developed for plume transmissivity measurements aimed at quantifying soot emission rates from unconfined industrial sources such as stack plumes and flares. In this approach, experimentally measured optical transmissivities through a soot containing sample are transformed into soot volume fraction data using the RayleighDebye-Gans scattering approximation in which soot aggregates are treated as polydisperse fractal aggregates. The new sky-LOSA technique was investigated experimentally under different sky conditions to determine achievable sensitivities and uncertainties. Final experiments were performed on unconfined soot plumes issuing into the open atmosphere to determine the ultimate accuracy and sensitivity limits in transmissivity measurements using sky-scattered radiation. The results showed that a minimum transmissivity through the centre of the plume of 0.995 could be analyzed. At a plume velocity of $12.5 \mathrm{~km} / \mathrm{hr}$ (corresponding to a typical average wind speed for locations in Alberta), the theoretical minimum mass flow rate of soot that could be detected in a $2 \mathrm{~m}$ wide plume is $3.5 \mathrm{mg} / \mathrm{s}, 4.4 \mathrm{mg} / \mathrm{s}$, and $3.4 \mathrm{mg} / \mathrm{s}$ for clear, cloudy, and overcast sky conditions, respectively. For a range of assumed field conditions, an uncertainty propagation analysis showed that overall uncertainties in $\dot{m}_{\text {soot }}$ of approximately $40 \%$ are achievable using sky-LOSA, where the most significant uncertainties arise from the estimation of plume velocity and soot optical properties. 


\section{Acknowledgements}

I wish to express the deepest gratitude to my supervisors, Dr. Matthew Johnson from Carleton University and Dr. Kevin Thomson from National Research Council Canada (NRC), who have given me invaluable guidance, inspiration, encouragement, patience and support throughout this journey. They are always ready to help me whenever I encounter a problem. I am strongly impressed by their knowledge in flaring combustion and optical diagnostics and their passion to explore and solve challenging problems. Without my supervisors this research would not have been possible. The experience and skills that I have accumulated under their counsel will be of value throughout my career.

In addition, I am grateful to Dr. William Hallett and Dr. Donald Gauthier, not only for being my committee but also for teaching me a variety of courses throughout the academic program. I would also like to extend special thanks to Dr. Amir Hakami for being my committee member.

I greatly appreciate NRC technical officers, especially Reg Smith, Bob Sawchuk, Faz Baksh, Dan Gareau, and Dan Clavel on their important hands-on assistance with NRC facilities throughout this research project.

Finally, I would like to thank my mother, my parents-in-law, my husband, and my brother for their understanding and support to make this research possible. 


\section{Table of Contents}

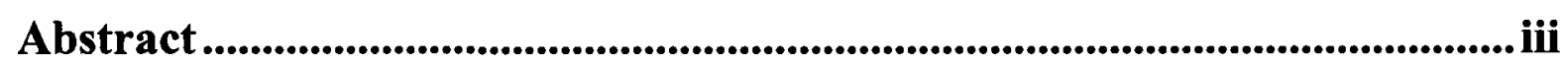

Acknowledgements............................................................................................................. iv

Table of Contents ............................................................................................................ v

List of Tables ..............................................................................................................viii

List of Figures........................................................................................................... ix

Nomenclature............................................................................................................... xiii

1 Introduction .............................................................................................. 1

$1.1 \quad$ Project Background and Challenges ................................................................ 1

1.1.1 Target Application of Solution Gas Flares .................................................. 3

1.2 Review of Existing Field Measurement Techniques ........................................... 4

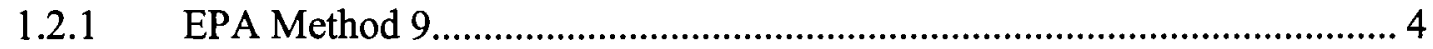

1.2.2 Digital Opacity Compliance System......................................................... 5

1.2.3 Remote Plume Opacity Sensing Technique.............................................. 7

1.2.4 Light Detection and Ranging Technique (LIDAR) and Differential Absorption LIDAR (DIAL) .............................................................................. 8

1.2.5 Line-of-Sight Attenuation Technique (LOSA or 2D-LOSA)................... 11

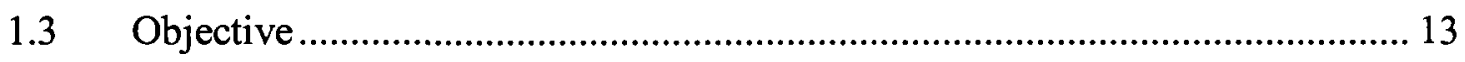

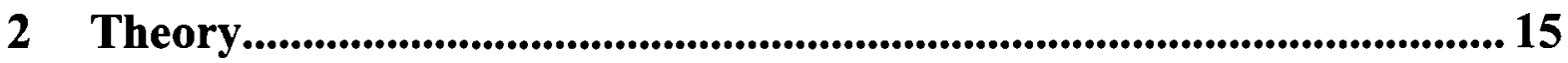

2.1 Soot Morphology .................................................................................. 15

2.2 Basic Scattering Theory - Light/Small Particle Interaction ............................. 18

2.2.1 Rayleigh Scattering and Absorption ...................................................... 19

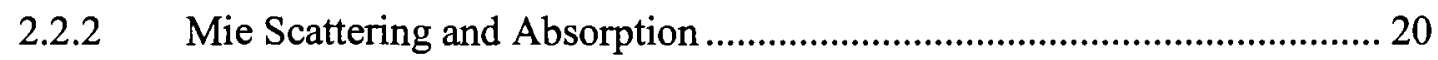

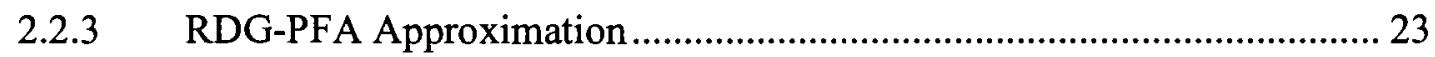

2.3 Two-Dimensional Line-Of-Sight Attenuation (2D-LOSA) Technique............. 28

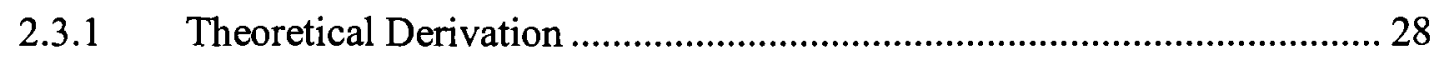


2.3.2 Use of 2D-LOSA to Estimate Soot Mass Emission Rate ........................ 32

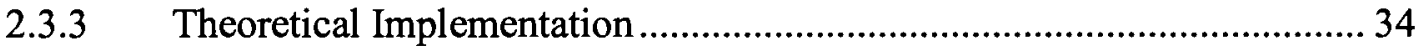

2.4 Scattering and Absorption of Light by Non-Soot Particles ........................... 37

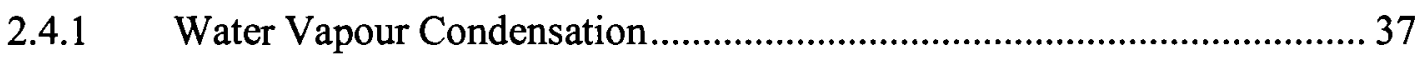

2.4.2 Sulfate and Nitrate Formation............................................................ 50

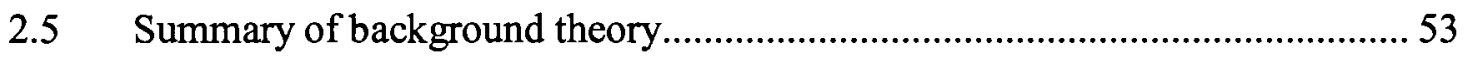

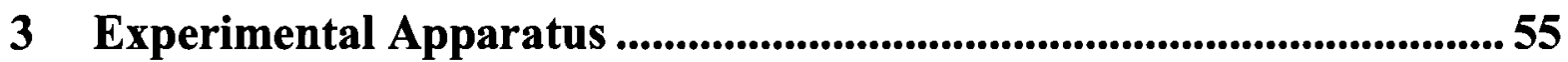

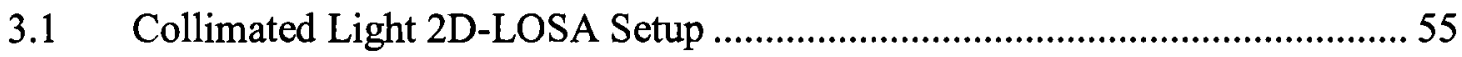

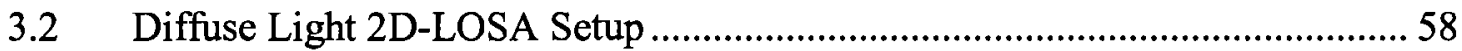

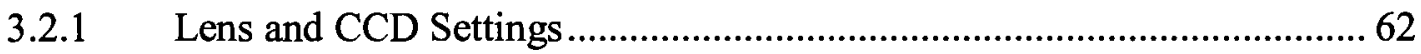

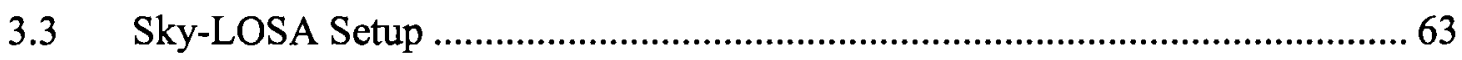

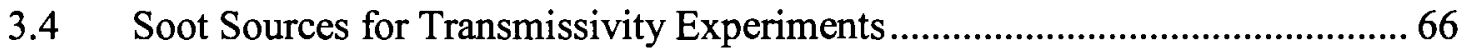

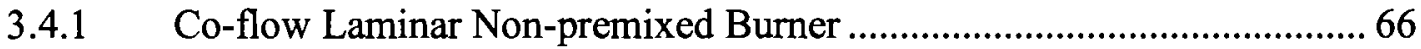

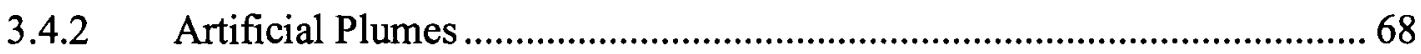

3.4.3 Inverted Co-flow Non-premixed Burner ........................................... 70

3.5 Summary of Experimental Apparatus.......................................................... 77

4 Results: Sensitivity and Uncertainty from Background Analysis.

4.1 Interpolation for Background Intensities Behind the Plume........................... 78

4.1.1 Background Interpolation Algorithm................................................... 78

4.1.2 Two Main Factors in the Background Interpolation Analysis: the Span

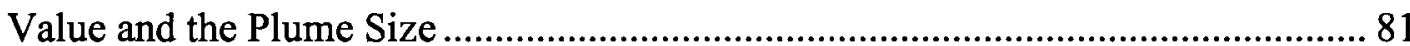

4.1.3 Quantification of Achievable Background Interpolation Uncertainties ... 86

4.1.4 Null Hypothesis Analysis on the Background Interpolation Bias ............ 90

4.1.5 Associated Errors in Soot Emission Rates from Background Interpolation Analysis 94

4.2 Additional sources of uncertainty in transmissivity measurements.................96

4.2.1 Nearest Limit of Object Distance and Associated Error......................... 96

4.3 Summary of background interpolation sensitivity and uncertainty ................ 99

5 Results: Sensitivity and Uncertainty of Plume Measurements........... 100

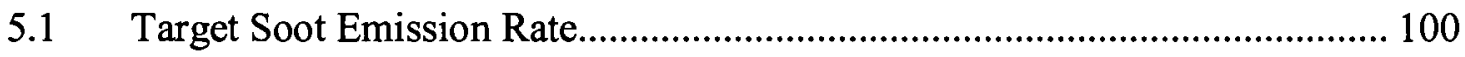

5.2 Results: Artificial Test Plume Experiments......................................... 102 


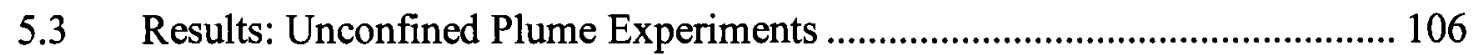

5.4 Total Uncertainty: Error Propagation Analysis .............................................. 111

5.5 Applicability of Sky-LOSA to Measuring Soot from Solution Gas Flares .... 114

6 Conclusions and Future Work ................................................................... 118

6.1 Conclusions ................................................................................................ 118

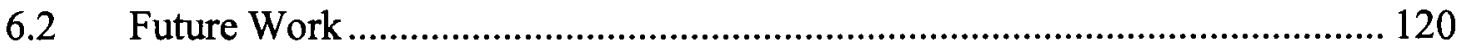

6.2.1 Direct Sunlight Scattering Effect ........................................................... 120

6.2.2 Wind Speed Measurement ....................................................................... 121

References .................................................................................................................. 122

Appendix A: Spatial Calibration for Field Application ............................ 132

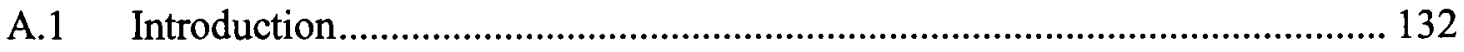

A.2 Field Magnification Calibration................................................................... 133

A.2.1 Plume Width and Object Distance Effects............................................. 139

A.2.2 Nearest Limit of Object Distance and Associated Error.......................... 142

Appendix B: Beam Steering .................................................................... 146 


\section{List of Tables}

Table 2.1: Estimated volume fraction of entrained air and products at a plume crosssection $110 \mathrm{~cm}$ downstream of the stack ......................................................... 40

Table 2.2: Comparison of estimated $T_{\text {plume }}$ with measured data ................................. 44

Table 2.3: Selected values for fraction of heat radiated from methane flames given in the

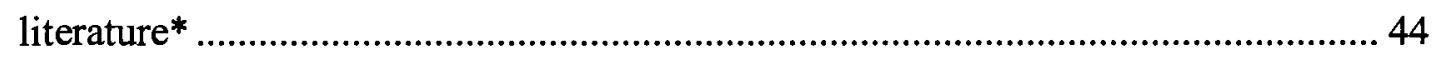

Table 2.4: Compare simulation results of plume temperatures with measured data ........ 46

Table 2.5: The estimation of $T_{\text {plume }}, P_{\text {total }}$, and $P_{\text {sat }}$ at the edge of the plume.................... 47

Table 3.1 Associated transmissivities of test plumes.............................................. 69

Table 3.2: Burner operating conditions corresponding to the plume transmissivities...... 74

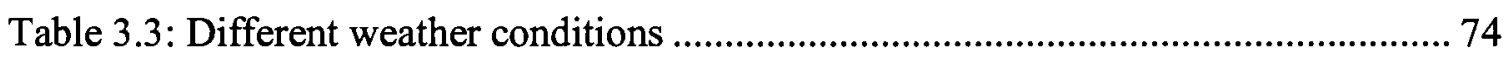

Table 4.1: Null hypothesis summary table for background interpolation biases under

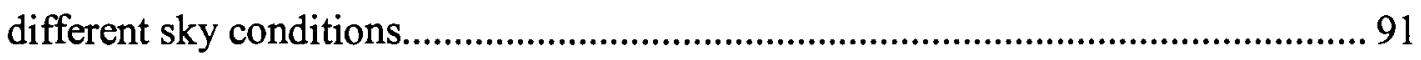

Table 5.1: Uncertainty analysis on unconfined plume experiments in the lab ............... 114

Table 5.2: Uncertainty analysis for assumed field measurement conditions using sky-

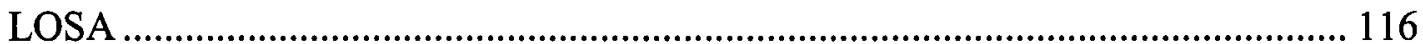




\section{List of Figures}

Figure 1.1: Use of the DOCS software: drawing of the opacity analysis box [McFarland et al., 2006].

Figure 1.2: Ideal LIDAR return signal from a smoke plume [Cook et al., 1972]

Figure 2.1: a) SEM image of soot aggregates sampled from the exhaust of a smoking methane/air diffusion flame [Coderre et al., 2007] b) TEM image of soot aggregates sampled from an ethylene/air diffusion flame [Tian et al., 2004].

Figure 2.2: Definition of the scattering angle, $\beta$.

Figure 2.3: Rayleigh scattering patter for polystyrene sphere of $D=50 \mathrm{~nm}$ in water. The incident laser light with $\lambda=632.8 \mathrm{~nm}$ [Morrison and Ross, 2002].

Figure 2.4: Angular dependence of scattered intensity for polystyrene sphere suspended in water a) $D=500 \mathrm{~nm} \mathrm{b)} D=2000 \mathrm{~nm}$. The incident laser light with $\lambda=632.8 \mathrm{~nm}$

[Morrison and Ross, 2002].

Figure 2.5: Scattering pattern for a sphere much larger than the wavelength of incident radiation [Siegel and Howell, 1992].

Figure 2.6: Transmission can be determined from the detector response with and without attenuating medium between source and detector.

Figure 2.7: Definition of coordinate directions for a plume transmissivity measurement.

Figure 2.8: Example of three-image method. The black line in the figure is an actual plume generated from a lab controlled burner.

Figure 2.9: Normalized $\mathrm{O}_{2}$ profile at the location of $110 \mathrm{~cm}$ downstream of the stack of a lab-scale flare in a wind tunnel. Crosswind velocity $2 \mathrm{~m} / \mathrm{s}$, plume exit velocity 1 $\mathrm{m} / \mathrm{s}$, and stack diameter $2.21 \mathrm{~cm}$ [Poudenx, 2000]. 39

Figure 2.10: Schematic diagram of a control volume

Figure 2.11 Total water vapour pressure of the plume and the saturation pressure at the plume edge temperature as a function of ambient air temperatures assuming ambient 
air saturated. A zoom-in figure between $-30^{\circ} \mathrm{C}$ to $-10^{\circ} \mathrm{C}$ is inserted to the original figure.

Figure 2.12: Total water vapour pressure and saturation pressure of the plume as a function of distance from the centre to the edge of the plume at ambient temperature of $-30^{\circ} \mathrm{C}$ with relative humidity of $100 \%$. A zoom-in figure between $10 \mathrm{~cm}$ to 15 $\mathrm{cm}$ is superimposed on the original figure.

Figure 3.1: Schematic of optical layout for Collimated LOSA setup................................. 57

Figure 3.2: Photograph of the Collimated LOSA setup. The yellow lines represent the collimated light path. A second arc lamp relates to a different setup and is not used for the collimated LOSA measurements. 58

Figure 3.3: Schematic of optical layout for Diffuse LOSA setup..................................... 59

Figure 3.4: Photograph of Diffuse LOSA setup 60

Figure 3.5: Initial and final configurations of source imaging lenses to improve collection ability of the whole system. In the final configuration, the angle $\theta_{1}$ of the light cone passing through the plume (in yellow) is much larger than the angle $\theta_{2}$ of the light cone collected by the imaging lens (in green).

Figure 3.6: Schematic (a) and photograph (b) of the integrating sphere ........................... 62

Figure 3.7: Schematic of optical layout for sky-scattered LOSA setup. ............................ 64

Figure 3.8: a) Sky-scattered light LOSA experimental setup b) another view of the same setup.

Figure 3.9: Co-flow laminar non-premixed burner. The schematic figure was adapted from [Thomson et al., 2005]

Figure 3.10: Soot source stability test for co-flow laminar non-premixed burner. 68

Figure 3.11: Artificial test plumes made by thermophoretically depositing soot onto microscope slides. Test plumes transmissivities a) $\tau_{\min }=0.4 \mathrm{~b}$ ) $\tau_{\max }=0.983$.

Figure 3.12: a) Schematic of the inverted co-flow burner b) Photograph of burner head 70

Figure 3.13: SMPS scans results at a burner condition of fuel $\left(\mathrm{CH}_{4}\right)$ flow rate of $1.2 \mathrm{slpm}$, co-flow air flow rate of $15 \mathrm{slpm}$, and dilution air flow rate of $50 \mathrm{slpm}$ in $\log$ scale [Coderre et al., 2007]. 
Figure 3.14: Transmissivities of plumes generated under different flow rates of fuel, coflow air, and dilution air. The transmissivities were determined using the in-lab diffuse 2D-LOSA diagnostic.

Figure 3.15: Number concentration normalized by its peak value vs. soot aggregate mobility diameter.

Figure 4.1: Comparison of interpolated and actual background for different light sources. Vertical dashed lines indicate region of interpolation. (a) Plots of absolute intensity and (b) normalized intensity. 80

Figure 4.2: Effect of smoothing parameter, span, in Loss function on background interpolation analysis

Figure 4.3: Background interpolation bias at different span values under various sky conditions for a) $20 \%$ of plume size, b) $30 \%$ of plume size, c) $40 \%$ of plume size, and d) $50 \%$ of plume size.

Figure 4.4: a) Clear b) Cloudy c) Overcast sky image. A) B) and C) Background interpolation biases corresponding to each sky condition.

Figure 4.5: Background interpolation bias under three sky conditions through the mirror.

Figure 4.6: Light intensity variation with horizontal position in image for different sky conditions. .94

Figure 4.7: Recommended distances of the observer from a plume for $20 \%$ plume size. 97 Figure 4.8: Associated errors in soot yield rates due to uncertainties in calibration for $20 \%$ plume size.

Figure 5.1: $\kappa=-\frac{1}{w} \int \ln \left(\tau_{\lambda}(y)\right) d y$ as a function of soot yield rate. Dashed line indicates target $4.2 \mathrm{mg} / \mathrm{s}$ soot emission rate estimated for solution gas flares. 102

Figure 5.2: a) comparison of 1 - and 3-image LOSA techniques for $\kappa$ with 3 light sources b) Zoomed-in plot without 1-image, collimated light data for artificial test plumes.

Figure 5.3: Measured uncertainties in $\kappa$ for test plumes using sky-LOSA and 1-image diffuse LOSA with 3-image diffuse LOSA as a standard a) uncertainty plotted vs. plume transmissivity and b) uncertainty plotted vs. $\kappa$ 
Figure 5.4: Comparison of 3-image diffuse LOSA and 1-image sky-LOSA under different sky conditions at the same test conditions after considering the biases in background interpolation analysis.

Figure 5.5: Detectable limits with sky-LOSA based on results of unconfined plume experiments assuming a $2 \mathrm{~m}$ diameter plume.

Figure A.1: Notation of terms...................................................... 134

Figure A.2: Schematic of the position of the target and the camera .....................136

Figure A.3: Schematic of the position of a plume and the camera.....................138

Figure A.4: Relative errors in magnification/ soot mass yield rate at different object distances from $10 \mathrm{~m}$ to $600 \mathrm{~m}$.

Figure A.5: Relative uncertainties in plume width for plume width from $1 \mathrm{~m}$ to $5 \mathrm{~m}$ at object distance a) from $10 \mathrm{~m}$ to $100 \mathrm{~m} \quad$ b) from $100 \mathrm{~m}$ to $600 \mathrm{~m}$.

Figure A.6: Recommended distances of the observer from a plume for $30 \%$ plume size.

Figure A.7: Associated errors in soot yield rates due to uncertainties in calibration for $30 \%$ plume size.

Figure B.1: Schematic beam steering through a circular plume.

Figure B.2: a) Beam steering $y_{\text {steer }}(x)$ simulation, $1 \mathrm{~m}$ diameter plume, Gaussian temperature profile. b) Corresponding image distortion. 


\section{Nomenclature}

\begin{tabular}{|c|c|}
\hline$a$ & Molar fractions of air in ambient air [dimensionless] \\
\hline$A$ & An instrument-related parameter [dimensionless] \\
\hline$A_{c s}$ & Plume cross-sectional area $\left[\mathrm{m}^{2}\right]$ \\
\hline$b$ & Molar fractions of $\mathrm{H}_{2} \mathrm{O}$ in ambient air [dimensionless] \\
\hline$c$ & Velocity of light $[\mathrm{m} / \mathrm{s}]$ \\
\hline$C_{\mathrm{a}}^{\lambda}$ & Absorption coefficient $[1 / \mathrm{m}]$ \\
\hline$C_{s}^{\lambda}$ & Scattering coefficient $[1 / \mathrm{m}]$ \\
\hline$C_{\mathrm{e}}^{\lambda}$ & Extinction coefficient $[1 / \mathrm{m}]$ \\
\hline$C_{\mathrm{a}}^{\mathrm{a}}$ & Absorption coefficient for aggregates same as $C_{\mathrm{a}}^{\lambda}[1 / \mathrm{m}]$ \\
\hline$C_{p}$ & Specific heat at constant pressure $[\mathrm{kJ} / \mathrm{kmol}-\mathrm{K}]$ \\
\hline$d$ & Molar fractions of $\mathrm{CO}_{2}$ in combustion products [dimensionless] \\
\hline$d_{\mathrm{p}}$ & Diameter of the primary particle $[\mathrm{m}]$ \\
\hline$D_{f}$ & Fractal dimension [dimensionless] \\
\hline$e$ & Molar fractions of $\mathrm{H}_{2} \mathrm{O}$ in combustion products [dimensionless] \\
\hline$E(m)_{\lambda}$ & Soot refractive index absorption function $=-\operatorname{Im}\left(\left(m^{2}-1\right) /\left(m^{2}+2\right)\right)$ \\
\hline$f$ & Molar fractions of $N_{2}$ in combustion products [dimensionless] \\
\hline$f_{\mathrm{v}}$ & Soot volume fraction [parts per million] \\
\hline $\bar{h}$ & Specific molar absolute enthalpy for each species $[\mathrm{kJ} / \mathrm{kmol}]$ \\
\hline $\bar{h}_{f}^{0}$ & Enthalpy of formation $[\mathrm{kJ} / \mathrm{kmol}]$ \\
\hline$\Delta \bar{h}$ & Sensible enthalpy change $[\mathrm{kJ} / \mathrm{kmol}]$ \\
\hline$I_{\lambda_{0}}$ and $I_{\lambda}$ & $\begin{array}{l}\text { Light intensity before and after passing through an attenuating } \\
\text { medium }\left[\mathrm{W} / \mathrm{m}^{3} \text { ster }\right]\end{array}$ \\
\hline$K_{f}$ & Fractal prefactor [dimensionless] \\
\hline$L$ & Effective pulse length \\
\hline
\end{tabular}




\begin{tabular}{|c|c|}
\hline$m$ & Complex refractive index [dimensionless] \\
\hline$\dot{m}_{\text {soot }}$ & Mass flow rate of soot $[\mathrm{kg} / \mathrm{s}]$ \\
\hline M & Magnification factor [dimensionless] \\
\hline$N$ & Number of primary particles in an aggregate $[\#]$ \\
\hline$N_{p}$ & Number of primary particles per unit volume $\left[\# / \mathrm{m}^{3}\right]$ \\
\hline$P_{r}$ & Return power (the received LIDAR signal) $[\mathrm{kJ}]$ \\
\hline$P_{t}$ & Outgoing laser power $[\mathrm{kJ}]$ \\
\hline$R_{g}$ & Cluster radius of gyration [m] \\
\hline$P_{w, \text { air }}$ & Water vapour pressure in ambient air $[\mathrm{Pa}]$ \\
\hline$P_{w, \text { comb }}$ & Water vapour partial pressure in the combustion products $[\mathrm{Pa}]$ \\
\hline$P_{\text {sat }}$ & Water saturation pressure $[\mathrm{Pa}]$ \\
\hline$P_{\text {total }}$ & Total water vapour partial pressure in the plume $[\mathrm{Pa}]$ \\
\hline$R$ & Distance from the scattering objects to the detector [m] \\
\hline$t$ & Time when the scatter signal is detected [s] \\
\hline$t_{0}$ & Time of the laser pulse starts $[\mathrm{s}]$ \\
\hline$T_{\text {ad }}$ & Adiabatic flame temperature $[\mathrm{K}]$ \\
\hline$T_{\text {air }}$ & Ambient air temperature $[\mathrm{K}]$ \\
\hline$T_{\text {plume }}$ & Final plume temperature $[\mathrm{K}]$ \\
\hline$u$ & Plume velocity $[\mathrm{m} / \mathrm{s}]$ \\
\hline$w$ & Width of a plume in the image $[\mathrm{m}]$ \\
\hline$x_{\mathrm{P}}$ & Primary particle size parameter [dimensionless] \\
\hline$x$ & Coordinate along the optical axis \\
\hline$y$ & Coordinate perpendicular to $x$ \\
\hline$z$ & Plume propagation direction \\
\hline$d x$ & Shift in the $x$-direction \\
\hline$d y$ & Shift in the $y$-direction \\
\hline$\alpha$ & Fraction of combustion products in a sample [dimensionless] \\
\hline$\alpha_{\mathrm{a}}^{\mathrm{P}}$ & Absorption cross-section of a primary particle $\left[\mathrm{m}^{2}\right]$ \\
\hline$\beta$ & Scattering angle $\left[{ }^{\circ}\right]$ \\
\hline
\end{tabular}


$\kappa$

$$
\rho_{\mathrm{sa}, \lambda}
$$

$\rho_{\text {soot }}$

$\tau_{\text {laser }}$

$\tau_{\lambda}$

$\lambda$

$\theta_{1}$

$\theta_{2}$

$\xi$

Acronyms

$\mathrm{A} / \mathrm{D}$

CCD

CCME

$\mathrm{CPC}$

DIAL

DMA

DOCS

EPA

IEFS

LIDAR

LOSA

2D-LOSA

Collimated LOSA

Diffuse LOSA

sky-LOSA

NAAQS

NPRI
Non-dimensional parameter (defined as $-(1 / w) \int \ln \left(\tau_{\lambda}(y)\right) d y$ )

(normalize the integration of the natural logarithm of the local transmissivity by the width of the plume)

Ratio of scattering to absorption coefficient [dimensionless]

Soot density $\left[\mathrm{kg} / \mathrm{m}^{3}\right]$

Laser pulse duration $[\mathrm{s}]$

Transmissivity [dimensionless]

Wavelength [m]

Collection angle of incident light $\left[{ }^{\circ}\right]$

Imaging angle of $\mathrm{CCD}$ at specific $\mathrm{F}-\#\left[^{\circ}\right]$

Particle size parameter [dimensionless]

Analog-to-Digital

Charge-coupled device

Canadian council of Ministers of the Environment

Condensation Particle Counter

Differential Absorption LIDAR

Differential Mobility Analyzer

Digital Opacity Compliance System

Environmental Protection Agency

Integral Equation Formulation for Scattering

Light Detection and Ranging Technique

Line-of-Sight Attenuation

Two-Dimensional Line-of-Sight Attenuation

Line-of-Sight Attenuation using collimated light source

Line-of-Sight Attenuation using diffuse light source

Line-of-Sight Attenuation using skylight source

National Ambient Air Quality Standards

National Pollutant Release Inventory 
PAH

PM

$\mathrm{PM}_{2.5}$

psig

RDG-PFA

SEM

sccm

SLPM

SMPS

TEM
Polycyclic Aromatic Hydrocarbons

Particulate Matter

Particulate Matter diameter less than $2.5 \mu \mathrm{m}$

Gauge pressure in units of pounds per square inch

Rayleigh-Debye-Gans scattering model for polydisperse fractal aggregate populations

Scanning electron microscope

Cubic centimeters per minute as standard temperature and pressure Standard litres per minute at $20^{\circ} \mathrm{C}$ and $101.3 \mathrm{kPa}$

Scanning mobility particle sizer

Transmission electron microscope 


\section{Introduction}

\subsection{Project Background and Challenges}

Particulate matter (PM) originates from a wide variety of stationary industrial sources. PM-laden plumes may be emitted into the atmosphere from stacks at plants, from exhaust ports of combustion devices, and from open flames such as gas flares or open fires. In many combustion systems, the most important component of PM is soot, which consists of carbon-rich branched-chain aggregates with a broad size distribution. Sulphate and nitrate particles can also be generated via combustion processes.

Since most particulates in ambient air are not spherical, quantification of their size is not trivial. A common size designation is the aerodynamic diameter. The aerodynamic diameter is an equivalent diameter that is used to compare the dynamic properties of particles of different shapes and/or densities. The aerodynamic diameter is the diameter

of the spherical particle of density $1000 \mathrm{~kg} / \mathrm{m}^{3}$ that has the same terminal speed as the particle of concern [Colls, 2002]. PM is classified in terms of the particle's aerodynamic diameter as follows:

- Nonrespirable fraction (between $50 \mu \mathrm{m}$ and $100 \mu \mathrm{m}$ )

- $\mathrm{PM}_{10}$ (smaller than $\left.10 \mu \mathrm{m}\right)$

- $\mathrm{PM}_{2.5}$ (smaller than $2.5 \mu \mathrm{m}$ )

- $\mathrm{PM}_{0.1}$ (smaller than $\left.0.1 \mu \mathrm{m}\right)$

$\mathrm{PM}_{2.5}$ is also referred to as fine particulate matter and $\mathrm{PM}_{0.1}$ is called ultra-fine particulate matter. The suspension of very small PM in the atmosphere has adverse short-term and 
long-term effects on human health. Recent scientific studies suggest that these effects are more closely linked with fine and ultra-fine particulates, because these particulates can easily penetrate deep into the lungs [Schwartz et al., 1999]. Epidemiological studies have shown consistent association between exposure to elevated levels of PM and increased respiratory health problems, hospitalization for heart and lung disease, and mortality [Pope et al., 2002, Peters et al., 2001]. Besides severe human health effects, fine particles have a wide range of impacts on the environment such as reduced visibility, soiling and material damage effects, and acid deposition [US EPA, 2004]. PM as soot is also a contributor to global warming and it could have as much as $60 \%$ of the current global warming effect of carbon dioxide [Ramanathan and Carmichael, 2008]. However, there is still significant uncertainty with the strength of climate forcing of PM and soot in the atmosphere, and the recent fourth assessment report from the Intergovernmental Panel on Climate Change suggests a more modest effect of $13-26 \%$ that of $\mathrm{CO}_{2}$ [IPCC, AR4, 2007].

For these reasons, in July 1997, the U.S. Environmental Protection Agency (EPA) announced the revised National Ambient Air Quality Standards (NAAQS) for PM to add new standards for fine particles $\left(\mathrm{PM}_{2.5}\right)$, as well as retaining standards for $\mathrm{PM}_{10}$ [US EPA, 1997]. In Canada, since 2002, facilities have been required to report PM emissions to the Canadian National Pollutant Release Inventory (NPRI). Further regulation of PM emissions is still being considered (or negotiated) at this time. For public health and environmental considerations, new and more stringent limitations on the emissions of PM from combustion processes are expected to be imposed by regulation. Tighter control of 
PM creates the need for reliable and accurate means to measure and report PM emission from various industrial sources.

\subsubsection{Target Application of Solution Gas Flares}

A significant source of PM emissions in Canada and internationally is from gas flares [CCME, 2005]. Flaring is a common method for disposal of flammable waste gases in the oil and gas industry. The flare is an open-air flame usually at the top of a long stack, exposed to the wind. Gases may be flared as part of the production in oil and natural gas fields or as an unwanted by-product of refining or chemical processing [Johnson et al., 2001]. The flaring process can produce significant amounts of a highly carbonaceous material known as soot, which is a major contributor to ambient particulate matter (PM). Although other forms of particulate such as sulphates and nitrates could be emitted or created in the plumes of flares, as will be discussed later on in this thesis, these are not expected to be significant in the near-field of the plume, close to the flame. This thesis will thus focus on developing a technique to quantify soot emissions from flares in the absence of other particulate species.

Previous work on emissions from turbulent diffusion flames for a range of fuels showed that the sizes of soot aggregates are generally less than $1 \mu \mathrm{m}$ [Koylu and Faeth, 1994a]. Although the author is not aware of direct measurements of soot in plumes of flares, it is reasonable to assume that the soot emission from flares are $\mathrm{PM}_{2.5}$.

The performance of flares in the field is difficult to quantify. Several challenges are faced that are not encountered from other soot sources. Wind speed and direction are highly variable while the flares can be quite high above the ground and in remote locations. Consequently, physical sampling, collecting combustion products, or any form 
of permanently mounted monitoring system is almost impossible. Furthermore, the composition and flow rate of flared gas may vary with time and location, making prediction of emissions difficult. These factors demonstrate the difficulties experienced when quantifying soot emission rate for stationary sources. In the next section, existing field measurement techniques are discussed.

\subsection{Review of Existing Field Measurement Techniques}

\subsubsection{EPA Method 9}

EPA Method 9 (i.e., Method 9) is a test method defined and regulated by the United States Environmental Protection Agency (US EPA) for visual determination of the opacity of emissions from stationary sources. Method 9 relies on a certified human observer to visually estimate the opacity of a plume emitted from a stack. The observer takes a series of opacity measurements at a rate of one every 15 seconds for a specified period of time. The collective set of opacity measurements then is averaged to develop a single opacity reading for the regulated source, which is compared to permitted levels [US EPA, 1971]. Plume opacity is the amount of light which is blocked by the plume and is usually stated as a percentage. An opacity of $20 \%$ means that $20 \%$ of incident light is absorbed, scattered, or otherwise blocked by the plume while $80 \%$ is transmitted. To qualify as an EPA Method 9- certified human observer, an individual must attend classroom training and successfully pass a visual opacity field examination conducted at an EPA-approved smoke school once every six months. Such requirements make Method 9 labour-intensive and expensive. Because the opacity is determined through visual observation by people, Method 9 has a highly subjective nature and the accuracy varies under different sky conditions [US EPA, 1975]. Furthermore, the method is non- 
quantitative since white light opacity measurements cannot be related to soot release rates. Although EPA Method 9 might best be described as an aesthetic rather than a quantitative standard, human observed measurements are also the default approach for monitoring opacity in Canada [Province of Alberta, 2005].

\subsubsection{Digital Opacity Compliance System}

A recently proposed alternative to Method 9 is the Digital Opacity Compliance System (DOCS), which was developed as an objective technique for measuring opacity [McFarland et al., 2006; McFarland and Terry, 2004 2003]. The DOCS technology employs digital photography in combination with proprietary analysis software to determine the opacity of a plume generated from a stationary source. The DOCS uses a digital camera to capture images of visible emission, which are then downloaded to a standard personal computer running the DOCS photographic imaging software. Once downloaded to a computer, the operator selects an area in the photograph which includes the part of the plume where opacity will be determined and the clear sky background. The area is called an analysis box and is shown in Figure 1.1 (adopted from McFarland et al., 2006). 


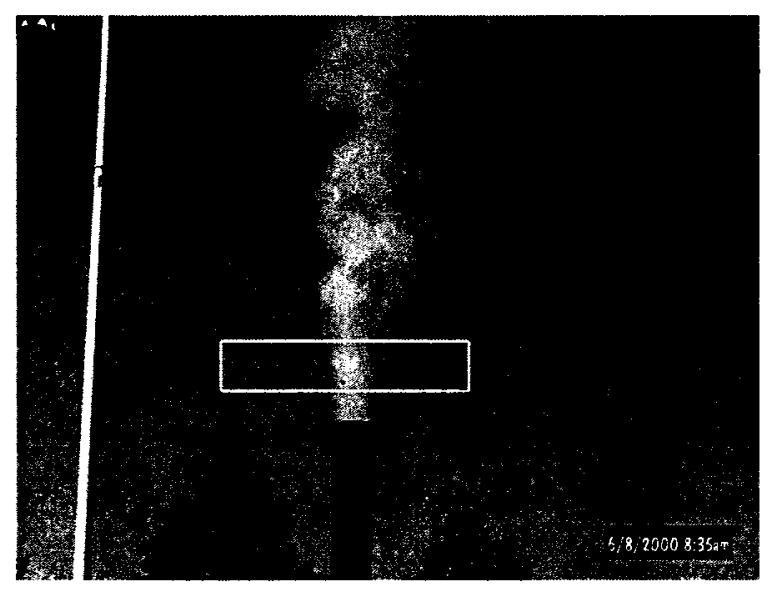

Figure 1.1: Use of the DOCS software: drawing of the opacity analysis box [McFarland et al., 2006].

After selection of the analysis box, the DOCS software distinguishes the visible emission from the background. By assuming that the pixels located at the side edges of the analysis box correspond to unobscured background and those located in the centre represent visible emissions, the software is able to determine if the emissions are lighter or darker than the background. The DOCS software begins calculation of the plume opacity by assigning the pixel intensity associated with the background to equal $0 \%$ opacity, then proceeds to evaluate the pixels within the analysis box to capture the spatial variation of intensity, and finally compares the contrast between the plumes and the selected background to generate an opacity reading. The size and shape of the analysis box which is controlled by the user must be chosen judiciously because the final opacity measurement will ultimately depend on what part of the image the DOCS software identifies as background. Based on the statistical analysis, the DOCS technology measures visible opacity with an accuracy of $1.12 \%$ (on average) greater than Method 9 certified human observers over the range of visible opacity from $0 \%$ to $40 \%$ [McFarland et al., 2006]. The ability of the DOCS to estimate plume opacity accurately could vary 
significantly depending on intensity contrast between the plume and sky conditions. If visual contrast between the plume and sky can not be readily established, DOCS had difficulty in accurately quantifying opacity [McFarland and Terry, 2004 2003].

\subsubsection{Remote Plume Opacity Sensing Technique}

Another method that uses skylight as a light source for visual opacity measurement is the remote smoke plume opacity sensing technique proposed by Lilienfeld et al. (1981). Lilienfeld et al. pointed out that, when using skylight as the source of light whose attenuation is to be measured, the intrinsic brightness of the plume resulting from the scattering of sunlight is a potential problem that interferes with the opacity measurement. To overcome this interference, the technique specifically measures the attenuation of the polarized component of the skylight which traverses a plume. To achieve this, the experiment is set up with a detection system which is filtered with a light polarizer. Measurements are made with the polarizer aligned parallel to the skylight polarization direction and with the polarizer rotated $90^{\circ}$. Opacity is derived from the difference between these two intensities. With this technique a few additional assumptions are required:

1) Clear skylight features partial plane-polarization.

2) The sunlight scattered by the plume in the direction of observation must be unpolarized.

3) The plume transmittance or opacity is independent of the wavelength.

It is noted that assumption 3 is not a reasonable assumption for soot aerosols because the transmittance is inversely dependent on the wavelength of incident light (see Equation 2.5 in Chapter 2). The applicability of this technique is further limited in the presence of haze which reduces the polarization of sky-scattered solar radiation. However, the 
concept mentioned by Lilienfeld et al. of the interference caused by sunlight directly scattered by the plume is potentially relevant to any diagnostic that uses background skylight as a source for attenuation measurements.

Both DOCS and Remote Plume Opacity Sensing measure the opacity of plumes emitted from smokestacks using skylight as a light source. The opacity is measured based on the attenuation of white (i.e., broad band) light transmission. As will be explained in Section 2.3.1, it is not possible to relate broadband opacity to quantitative particulate concentration or particulate mass emission rate and so neither are appropriate as a method to quantify the particulate emissions from flares.

\subsubsection{Light Detection and Ranging Technique (LIDAR) and Differential Absorption LIDAR (DIAL)}

LIDAR is an acronym for light detection and ranging. LIDAR systems operate on a similar principle as radar (radio detection and ranging), but use a pulsed laser as a source of energy. In a typical LIDAR system, periodic high power laser pulses are sent out toward the region of interest in the atmosphere through a laser transmitter. The laser light is backscattered from the atmosphere and collected by a large diameter telescope. Behind the telescope, the light passes through a filter and a lens system and is focused on a photo detector with a high temporal resolution. The signal is amplified as part of the detector system and fed into a digitizer.

The backscattered (return) power received by the detector, $P_{r}(R)$, is a function of the outgoing laser power, $P_{t}$, as characterized by the following equation [Collis and Uthe, 1972]: 


$$
P_{r}(R)=\frac{P_{t} L C_{s}^{\lambda}(R) A}{R^{2}} \exp \left[-2 \int_{0}^{R} C_{a}^{\lambda}(r) d r\right]
$$

where $P_{r}(R)$ is the return power (the received LIDAR signal); $P_{t}$ is outgoing laser power at $t_{0} ; L$ is the effective pulse length ( $L=c \tau_{\text {laser }} / 2$ where $c$ is the velocity of light and $\tau_{\text {laser }}$ is the laser pulse duration); $R$ is the distance from the scattering objects to the detector $\left(R=c\left(t-t_{0}\right) / 2\right.$, where $t$ is the time when the scatter signal is detected and $t_{0}$ is the time of the laser pulse starts); $A$ is an instrument-related parameter; $C_{s}^{\lambda}$ and $C_{a}^{\lambda}$ are the volume backscattering and absorption coefficients of the atmosphere.

The LIDAR technique was first used for air pollutant studies in the 1970s. Collis and Uthe (1972) suggested that the plume opacity measurements could be made by observing backscattered signal return from the near and far side of the plume through the ambient atmosphere. Cook et al. (1972) further explored the possibility of using the LIDAR technique for making a remote transmittance measurement, as illustrated qualitatively in Figure 1.2 below, by comparing the intensity of backscattered laser light before and after the plume. The returned signal from the clear air between the laser and the plume diminishes continuously. When the laser pulse intercepts the plume, the backscattered signal may increase sharply above the ambient atmospheric scattering due the presence of soot which is a strong scatterer, appearing as a spike on the LIDAR signal. The signal from just in front of the plume (B) and the signal from just beyond the plume (A) are measured as indicated in Figure 1.2. The measured discontinuity is related to the transmissivity of the plume. 


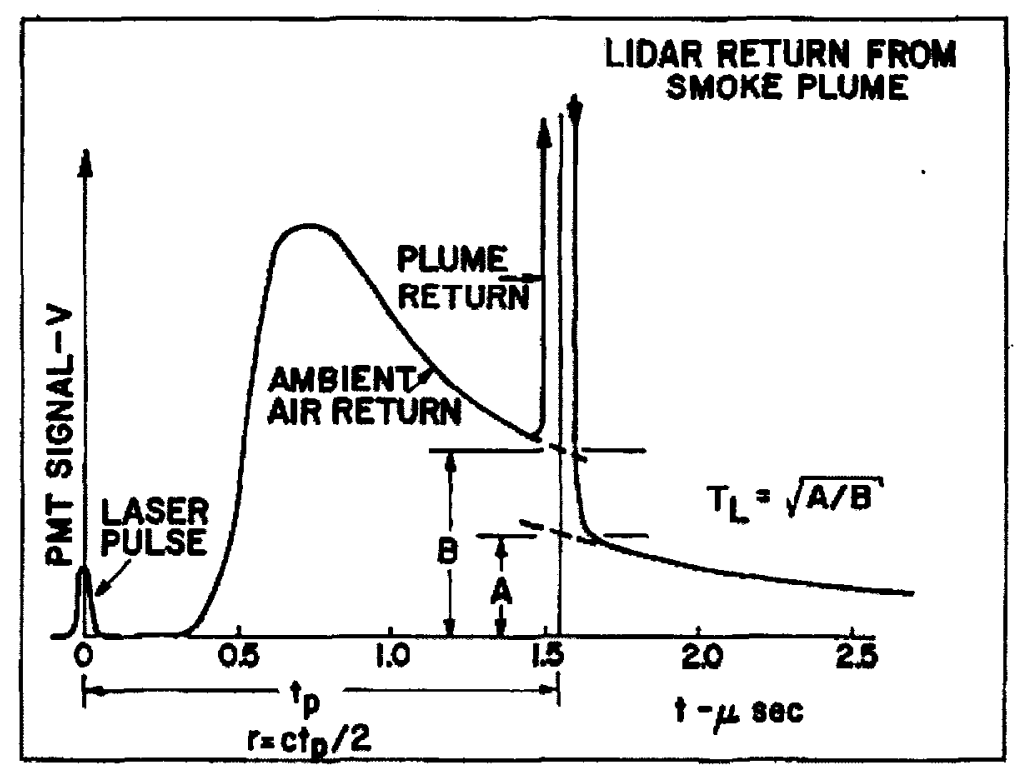

Figure 1.2: Ideal LIDAR return signal from a smoke plume [Cook et al, 1972]

However, in practice, measurements have shown that the intense backscattering signal from the plume itself can cause the system's amplifying electronics to be driven hard enough to produce a slow signal recovery in the region following the plume [Cook et al., 1972]. This delay adversely influences the extrapolation of signal value of " $\mathrm{A}$ ", contributing to an error in plume transmissivity evaluation. Due to this inherent problem, most recent applications of LIDAR have focused on measuring concentrations of specific molecular species (gaseous air pollutant in a plume) using a differential absorption LIDAR (DIAL).

For differential absorption LIDAR, two laser pulses with different wavelengths are selected. One is the measurement wavelength, which falls on an absorption line of the species of interest. The second is the reference wavelength for which the absorption cross-section of the species of interest is much weaker or negligible. The concentration of measured species is derived from the difference of the light intensities at the two wavelengths. Zhao et al. (2002) employed DIAL to measure ammonia concentration in a 
plume from a point source. Schroter et al. (2003) investigated $\mathrm{SO}_{2}$ emissions by applying DIAL. However, for the complex composition of a plume and specifically for the quantification of soot concentration, LIDAR has limitations. Since soot has a continuous absorption across all wavelengths and does not have a spectrally selective absorption cross-section [Johnson and Thomson, 2005], no wavelength exists for which a reference transmission can be recorded. Furthermore, the problem of scattering interference still exists for DIAL measurements. Finally, the high power laser and high temporal resolution detector place high demands on the system, leading to high costs and complexity.

\subsubsection{Line-of-Sight Attenuation Technique (LOSA or 2D-LOSA)}

Line-of-sight attenuation (LOSA) is a well-established, non-intrusive optical diagnostic for line-averaged measurement of soot concentration in laminar diffusion flames. The key difference between LOSA and opacity measurements is that the LOSA technique is done with monochromatic light, which enables the use of light/particle interaction theory to extract a quantitative measurement.

The two-dimensional line-of-sight attenuation (2D-LOSA) technique is an implementation of LOSA in which an extended light source and two-dimensional detector array are used to allow multiple parallel LOSA measurements at a single time. The technique was first performed with an expanded laser beam as the light source by Greenberg and $\mathrm{Ku}$ (1997). When applied to a two-dimensional soot field in combination with inversion algorithms [Dasch, 1992; Snelling et al. (1999); Daun et al., 2006], it is possible to measure spatially resolved soot concentrations. Snelling et al. (1999) improved the spatial resolution of the technique by using a collimated light source 
generated by an arc lamp (referred as collimated LOSA) to reduce the acquisition time and avoid the spectral coherence of laser sources.

Most recently, Thomson et al. (2008a) developed diffuse-light 2D-LOSA in which an arc lamp coupled with an integrating sphere is used as the extended light source. Diffuse LOSA differs from collimated LOSA in that diffuse light is used as the extended source. The technique offers advantages over collimated LOSA because it is less sensitive to defects in the optics and interferences due to beam steering, thus achieving very high levels of sensitivity in transmissivity measurements [Thomson et al., 2008a]. A detailed analysis of beam steering in LOSA measurements can be found in Appendix B.

Most lab-based LOSA systems have a signal sending device (i.e., lamp or laser) and a receiver (i.e., camera). However, in the field it is not practical to mount a signal sending device in the air beyond the plume because smoke stacks can be quite high above the ground in remote locations. As with the other techniques described above, skyscattered solar radiation offers an extended source which can be used to advantage in optical measurements. Therefore, a goal of this project was to develop a line-of-sight attenuation diagnostic that uses skylight as the light source, referred as sky-LOSA. This coincides well with the lab-based Diffuse 2D-LOSA [Thomson et al., 2008a] because the skylight is in essence a diffuse light source. It is worth pointing out that this skylightbased diagnostic differs from the others mentioned above because monochromatic light is used which is necessary for quantitative measurements. In Chapter 2 quantitative determination of soot emission from plumes using sky-LOSA is explained. 


\subsection{Objective}

A literature review has shown that qualitative soot measurement methods have been studied extensively. However, there is no accepted quantitative approach for estimating soot emissions from unconfined sources, such as industrial plumes and flares. This project focuses on developing a sky-scattered solar radiation-based optical diagnostic to quantify soot emission rates from plumes/flares with known sensitivity and uncertainties. The specific tasks necessary to accomplish this objective are described below.

1. Modify a lab-based optical diagnostic for outdoor application where very subtle light attenuations must be measured (i.e., transmissivity very close to 1) using sky-scattered light as the extended light source.

2. Develop image post-processing algorithms to interpret the skylight transmission images in order to calculate the plume transmissivity image by image.

3. Test and create steady soot sources for trial plume attenuation measurements.

4. Compare different light sources for plume transmissivity measurements

5. Evaluate the sensitivity limit of plume transmissivity measurement with the skyLOSA diagnostic.

6. Investigate the uncertainty in soot emission rate quantification using sky-LOSA under different sky conditions.

A complete description of the 2D-LOSA technique, including the theory of particle and light interaction and the relation between light transmission measurements and particle emission rate are described in Chapter 2. In Chapter 3, the optical experimental setups for different light sources and three types of lab-controlled synthetic plumes are 
described. A unique analysis method created for sky-LOSA technique tested with different sky conditions is discussed in Chapters 4. Chapter 5 presents experimental results of transmissivity measurements under different light sources and calculations of sensitivity limits for sky-LOSA measurements. Finally, the conclusions and future work are summarized in Chapter 6. 


\section{Theory}

This chapter presents a detailed overview of the theory behind the experimental techniques and methodology used to achieve the research goals of this project. As noted in Chapter 1, the focus of this project is to develop a technique to quantify the mass flow rate of soot emitted from solution gas flares. To discuss the measurement of soot it is first necessary to qualify what constitutes soot. This is presented in Section 2.1 . The interaction of light with soot can be quite complex because the particles in soot are typically smaller than the wavelength of light used to interrogate them. The theory and equations used to describe this interaction are introduced in Section 2.2. An explanation of the Line-of-Sight Optical Attenuation (LOSA) technique, the theoretical development of the relationship of soot transmissivity to the mass flow rate of soot for the target application of a gas flare, and the theoretical implementation of LOSA are presented in

Section 2.3. A key assumption of the technique is that soot is the only thing that attenuates the light. If other non-soot particles are present in the plume, they could interfere with the measurements. The potential for production of other light attenuating particles in the plume of a solution gas flare is discussed in Section 2.4.

\subsection{Soot Morphology}

The soot formation process in a flame is very complex as a result of the interacting processes of soot nucleation, growth and coagulation, and oxidation [Bockhorn, 1994; 
Glassman, 1996, Gülder, 1999; and Frenklach, 2002]. The soot formation process starts with hydrocarbon fuel undergoing pyrolysis into small hydrocarbon radicals from which polycyclic aromatic hydrocarbons (PAHs) are formed. The coagulation of large PAHs forms primary particles. These primary particles quickly grow by surface growth from reactions with gas phase molecules [Bockhorn, 1994]. As well, through reactive particleparticle collisions, primary particles evolve into soot aggregates by coagulation and agglomeration [Glassman, 1988, Frenklach, 2002]. Coagulation occurs when colliding particles fuse, forming larger particles, while agglomeration leads to the formation of irregular chain-like open structured aggregates [Bockhorn, 1994]. In late stages of the soot formation process, oxidation reactions take place on the surface of soot particles, depleting the carbon mass accumulated in the soot particles. The final amount of soot emitted from a flame is the result of the competition between soot growth and oxidation.

Numerous SEM (scanning electron microscope) and TEM (transmission electron microscope) photographs of soot aggregates at various flame conditions have appeared in the literature, all indicating a generic morphology or structure. Please see Faeth and Koylu (1995), Tian et al. (2004), and Coderre et al. (2007) for examples. Typical SEM and TEM images of soot aggregates appear in Figure 2.1. 

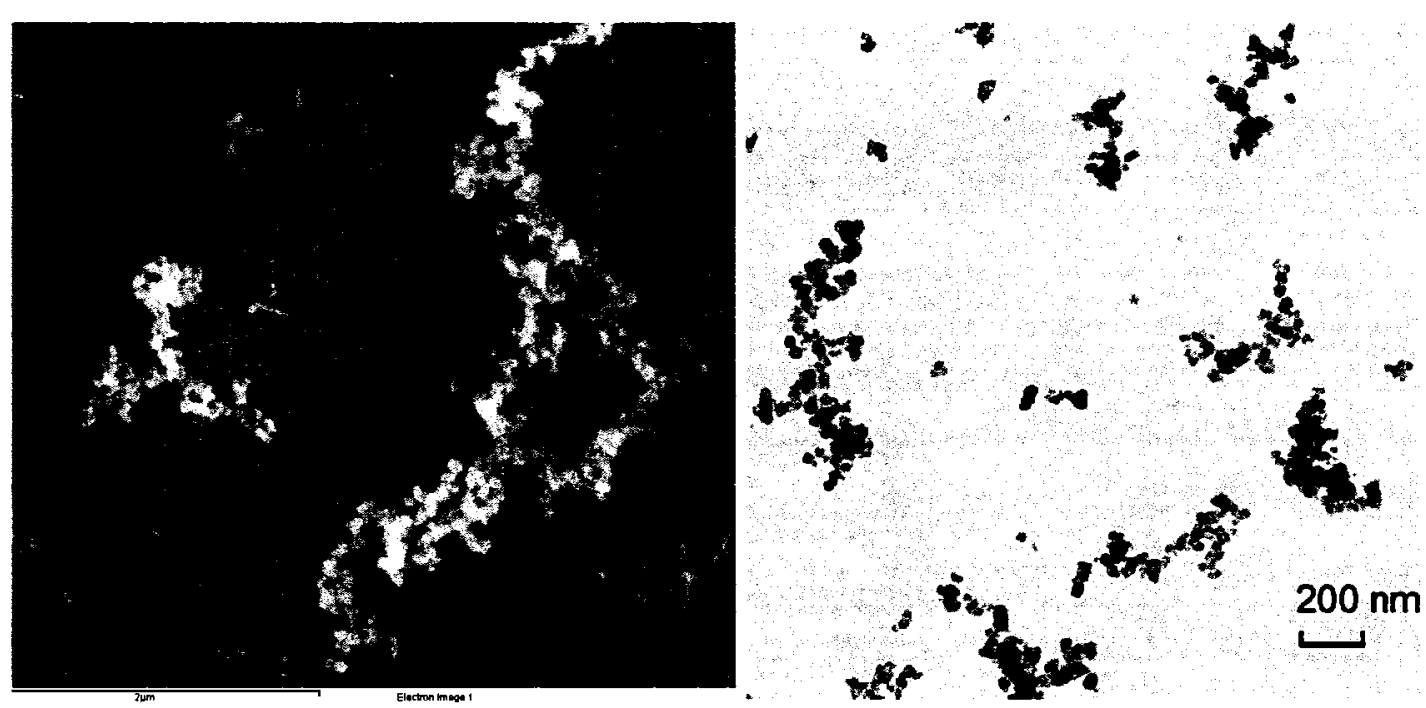

Figure 2.1: a) SEM image of soot aggregates sampled from the exhaust of a smoking methane/air diffusion flame [Coderre et al., 2007] b) TEM image of soot aggregates sampled from an ethylene/air diffusion flame [Tian et al., 2004].

Through quantitative examination of soot aggregates, Faeth and Koylu (1995) summarized the soot morphology and optical properties. They recognized that soot consists of nearly spherical primary particles. The size of primary particle varies with flame conditions and fuel type, with the largest particles being associated with the most heavily sooting fuels. Mean primary particle diameters less than $60 \mathrm{~nm}$ were observed in turbulent flames of various hydrocarbon fuels. Primary particles collect into branch-like and open-structured aggregates with a wide range in number of primary particles per aggregate. The distribution of the number of primary particles in soot aggregates can be approximated with a logarithmic normal distribution.

A fundamental relation to describe a fractal aggregate of $N$ primary particles [Jullien and Botet, 1987] is:

$$
N=K_{\mathrm{f}}\left(R_{\mathrm{g}} / d_{\mathrm{P}}\right)^{D_{\mathrm{f}}}
$$


where $N$ is the number of primary particles in an aggregate, $K_{\mathrm{f}}$ is the fractal prefactor, $R_{g}$ is the cluster radius of gyration (a root-mean-square distance of individual primary particles from the centre of mass of the aggregate), $d_{\mathrm{p}}$ is the diameter of the primary particles, and $D_{\mathrm{f}}$ is the fractal dimension (or Hausdorff number [Hausdorff, 1919]). The fractal dimension is a key parameter for the fractal description that quantifies the openness of the cluster aggregate. $D_{\mathrm{f}}=1.0$ corresponds to a straight line aggregate geometry. $\quad D_{\mathrm{f}}=3.0$ corresponds to compact clusters. For soot aggregates, the fractal dimension $\left(D_{\mathrm{f}}\right)$ is typically about 1.8 and is remarkably insensitive to flame conditions and fuel type [Wu et al., 1997].

\subsection{Basic Scattering Theory - Light/Small Particle Interaction}

When a beam of light illuminates a particle, the light scattered and absorbed by the particle depends on the size, shape, and material composition of the particle. Scattering can also depend on the wavelength and intensity of the incident radiation and the scatter angle, which is defined in Figure 2.2. In physical descriptions of scattering, physicists commonly distinguish between two broad types, elastic and inelastic. Elastic scattering involves no (or a very small) loss or gain of radiant energy, whereas inelastic scattering does involve some change in the radiant energy. Major forms of elastic light scattering (involving negligible energy transfer) are Rayleigh scattering and Mie scattering. Inelastic scattering effects include Brillouin scattering and Raman scattering [Bohren and Huffman, 1983]. Most scattering of importance in engineering is essentially elastic, and the discussion here will consider only the elastic case in which the frequency of the scattered light is the same as that of the incident light. 


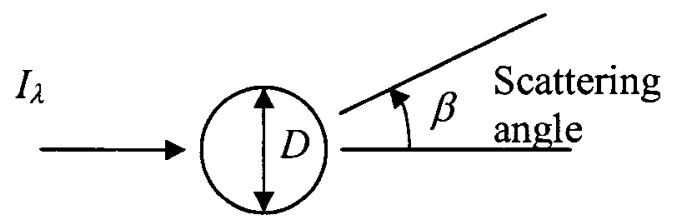

Particle with

diameter $D$

Figure 2.2: Definition of the scattering angle, $\beta$

\subsubsection{Rayleigh Scattering and Absorption}

Scattering involving particles much smaller than the wavelength $(\lambda)$ of the scattered light is known as Rayleigh scattering after British physicist Lord Rayleigh (1871) [Hecht, 2006]. Rayleigh found that the scattered energy in any direction is proportional to the inverse fourth power of the wavelength of the incident radiation [Siegel and Howell, 1992]. Therefore, Rayleigh scattering is wavelength dependent, with shorter wavelengths being more scattered. The Rayleigh scattering pattern is shown in Figure 2.3 (adapted from Morrison and Ross, 2002). It is observed that the intensity of the scattered light is uniform in all direction.

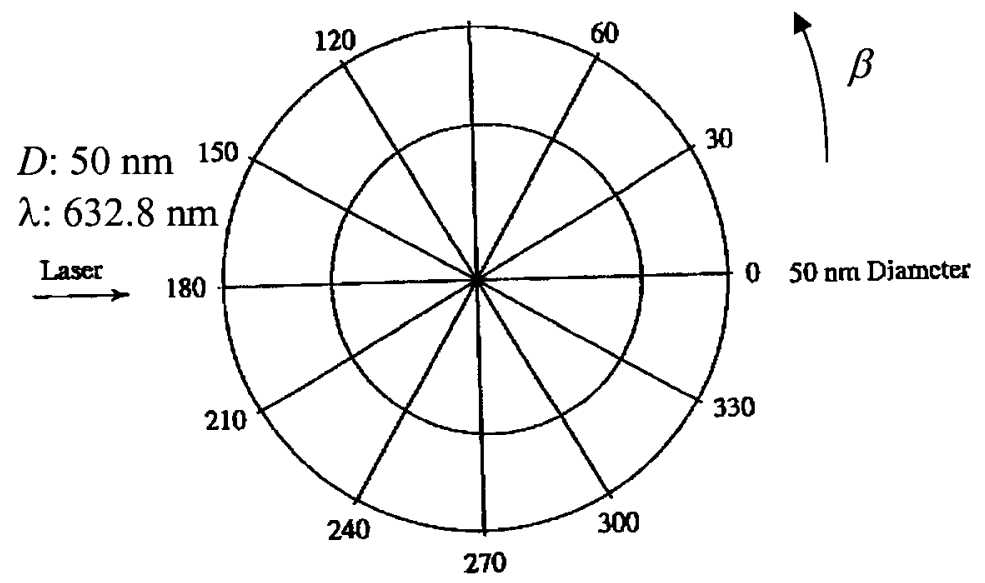

Figure 2.3: Rayleigh scattering patter for polystyrene sphere of $D=50 \mathrm{~nm}$ in water. The incident laser light with $\lambda=632.8 \mathrm{~nm}$ [Morrison and Ross, 2002]. 
The particle size relative to the wavelength is expressed as a particle size parameter, $\xi=\pi D / \lambda$ where $D$ is the spherical particle diameter. An approximate size limit for Rayleigh scattering is $\xi<\sim 0.3$ [Siegel and Howell, 1992,]. The primary particles which make up soot aggregates interrogated with visible light fall within the Rayleigh limit.

In the Rayleigh limit, the absorption cross-section of a primary particle, $\alpha_{\mathrm{a}}^{\mathrm{p}}$, is expressed as

$$
\alpha_{\mathrm{a}}^{\mathrm{P}}=\frac{\pi^{2} d_{\mathrm{p}}{ }^{3} E(m)_{\lambda}}{\lambda}
$$

where $d_{\mathrm{p}}$ is the primary particle diameter, $\lambda$ is the wavelength of the incident light, and $E(m)_{\lambda}$ is the soot refractive index absorption function which is discussed in Section 2.3.1. It is important to note that $\alpha_{\mathrm{a}}^{\mathrm{P}}$ is proportional to the volume of the particle. For this reason light absorption of particles which are in the Rayleigh limit (i.e., $\xi<0.3$ ) is proportional to the volume of the particles and can be used to measure the volume, concentration, or mass of the particles. Though the primary particles fall within the Rayleigh limit, soot aggregates are typically too large to be Rayleigh scatterers and thus other light/particle interaction theories must be considered.

\subsubsection{Mie Scattering and Absorption}

Mie scattering occurs from relatively large particles with dimensions comparable to the wavelength of the incident radiation. The approximate size range is $0.3<\xi<5$ [Siegel and Howell, 1992]. The theoretical analysis of scattering from spherical particles of any size was first published by German physicist Gustav Mie in 1908, from whom it gets its name [Hecht, 2006]. In the Mie regime, scattering is not strongly wavelength dependent 
but is angularly dependent. The shape of the particles becomes more significant and the theory only applies well to spherical particles. The resulting scattered radiation is nonuniform, varying strongly with the angle of scatter, $\beta$, and tends to be projected forward from the light source [Tsang et al., 2000]. Figure 2.4 shows the angular dependence of the scattered intensity for two different ratios of particle size to the wavelength of incident light (adapted from Morrison and Ross, 2002). Mie scattering is always strongest in the forward direction and this tendency is exaggerated for larger particles. One common example of Mie scattering is an interaction of visible light with water droplets. Since the size of water droplets are generally much larger than the wavelength of the visible radiation, droplets scatter all wavelengths nearly equally, causing them to appear white [Hecht, 2006]. 

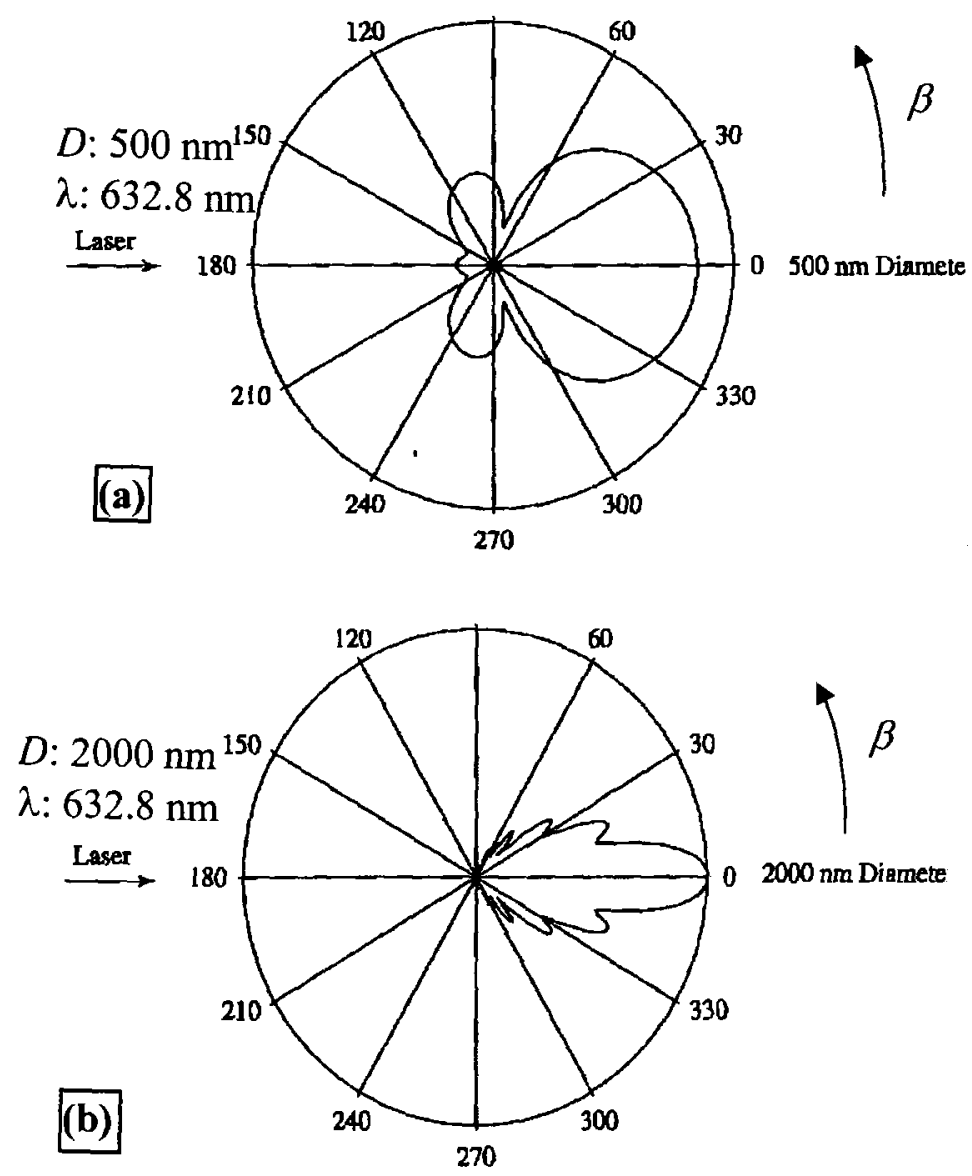

Figure 2.4: Angular dependence of scattered intensity for polystyrene sphere suspended in water a) $D=500 \mathrm{~nm}$ b) $D=2000 \mathrm{~nm}$. The incident laser light with $\lambda=$ $632.8 \mathrm{~nm}$ [Morrison and Ross, 2002].

The equations which describe the scatter and absorption of light for particles in the Mie regime are significantly more complex than that for the Rayleigh limit. A particular consequence of the Mie regime is that the assumption that light absorption is proportional to particle concentration no longer holds.

For large spheres, $\xi>5$, the scattering is mainly a reflection process and can be calculated from geometric reflection relations [Siegel and Howell, 1992]. Figure 2.5 illustrates the backward scattering pattern for a sphere large compared with wavelength of incident light. 


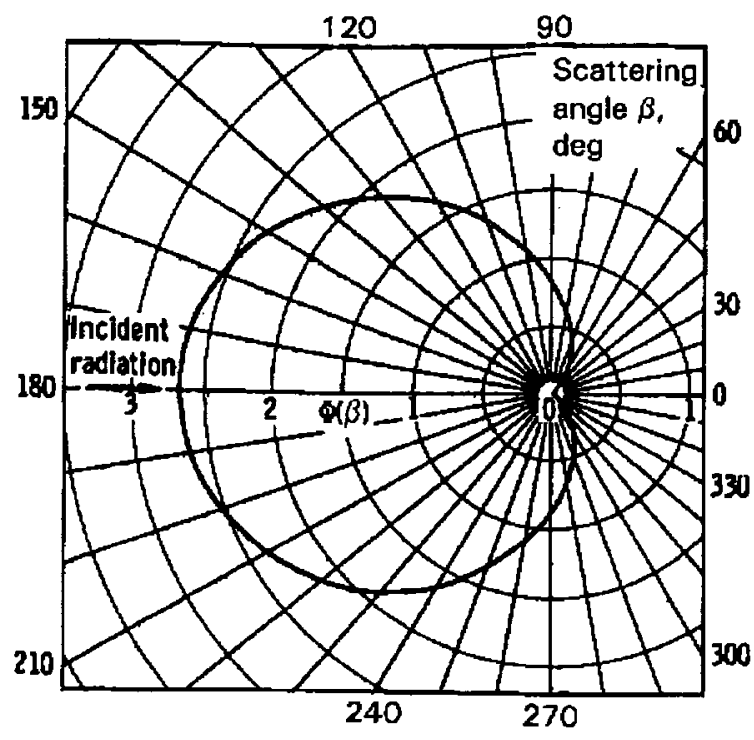

Figure 2.5: Scattering pattern for a sphere much larger than the wavelength of incident radiation [Siegel and Howell, 1992].

\subsubsection{RDG-PFA Approximation}

As discussed in the Soot Morphology section above, soot consists of small spherical primary particles with diameters of the order $30-60 \mathrm{~nm}$, which generally satisfy the Rayleigh scattering approximation. However, the primary particles collect into fractallike open structure aggregates during the soot formation, oxidation, and coagulation process to yield broad aggregate size distributions [Faeth and Koylu, 1995].

Koylu and Faeth (1994) measured the optical properties of overfire soot from turbulent diffusion flames for a variety of fuels. They found the optical properties of soot at $514.5 \mathrm{~nm}$ departed significantly from Rayleigh scattering behaviour: strong forward scattering was observed which is not representative of the Rayleigh approximation. Quinten et al. (2001) simulated light scattered from particle aggregates formed by identical spherical particles and compared this with scattered intensities from Mie theory for spheres with equivalent volume diameter to the aggregates. They found that the 
agglomerates scatter the light more efficiently than a volume-equivalent sphere. Therefore, the scattered light intensity increases due to shape irregularities caused by agglomeration. Thus, it was concluded that the optical properties of soot aggregates are not well described by Rayleigh or Mie scattering approximations. This has led to the development of an approximate Rayleigh-Debye-Gans (RDG) scattering model for polydisperse fractal aggregate (PFA) populations of soot [Faeth and Koylu, 1995].

Krishnan et al. (2000 and 2001) studied scattering properties for soot emitted from turbulent diffusion flames fueled with various gaseous and liquid hydrocarbon fuels at wavelength of $250-5200 \mathrm{~nm}$. They demonstrated that the measured scattering patterns of soot were in good agreement with predictions based on the Rayleigh-Debye-Gans scattering approximation in the visible range. Wang and Sorensen (2002) tested scattering of two fractal aggregate aerosols $\mathrm{SiO}_{2}$ and $\mathrm{TiO}_{2}$ at a wavelength of $488 \mathrm{~nm}$. They also found good agreement between the RDG prediction and experiments for fractal aggregates.

When using the RDG-PFA model, the major assumptions noted by Faeth and Koylu (1995) concerning soot physical properties are as follows:

- Individual primary particles are spherical and have constant diameters,

- Primary particles just touch one another and they are homogeneous with uniform refractive indices,

- The aggregate size distributions follow a log-normal distribution (although it is possible to assume other distribution shapes such as a self-preserving distribution),

- Effects of multiple- and self-scattering of soot aggregates are ignored, and 
- Light absorption by primary particles is not affected by aggregation.

Several investigators have explored the capability of the RDG-PFA theory to estimate optical properties of aggregates, finding encouraging performance within uncertainties involved in scattering / extinction experiments [Koylu and Feath, 1994a 1994b; Sorensen et al., 1992 2001; Wang and Sorensen, 2002].

A more rigorous evaluation of the RDG-PFA approximation was performed by Farias et al. (1996). They performed numerical experiments to test the validity of the RDG approximation in comparison with the exact scattering solution for a wide range of fractal aggregates. The exact scattering solution was calculated from the Integral Equation Formulation for Scattering (IEFS), a numerical method that satisfies the optical theorem, i.e., the sum of the total scattering and absorption cross sections is exactly equal to the extinction cross section of an aggregate. Additional details about the IEFS theory can be found in Ku and Shim (1992) and Lou and Charalampopoulos (1994). The IEFS is the most theoretically sound and elegant method; however, it is computationally intensive for treating polydisperse aggregates and it requires a priori knowledge of the primary particle position within each aggregate to compute the optical cross sections [Farias et al., 1996]. Farias et al. compared RDG-PFA predictions to IEFS and evaluated the percent differences between both scattering theories in the $x_{P}|m-1|$ domain for various size aggregates, where $m$ represents the complex refractive index and $x_{\mathrm{P}}$ is the primary particle size parameter and defined as

$$
x_{\mathrm{P}}=\pi d_{\mathrm{P}} / \lambda,
$$


As before, $d_{\mathrm{p}}$ is the diameter of the primary particles and $\lambda$ is the wavelength of light. For a soot aggregate, $m=1.57-0.56 \mathrm{i}$ [Smyth and Shaddix, 1996], corresponding to $|m-1|$ approximately equal to 1 [Farias et al., 1996].

The term $x_{P}|m-1|$ is the phase shift parameter for an aggregate and a condition for applicability of RDG-PFA is usually stated as

$$
2 x_{\mathrm{P}}|m-1|<<1 .
$$

Substituting Equation 2.3 into Equation 2.4, yields

$$
\frac{2 \pi d_{\mathrm{p}}|m-1|}{\lambda} \ll<1
$$

The quantity of $2 \pi d_{\mathrm{p}}|m-1| / \lambda$ represents, for a particle with a diameter of $d_{\mathrm{p}}$, the difference in phase between a light ray passing through the particle and a ray passing through the surrounding medium. If this quantity is much less than unity, the phase shift is negligible between the incident light that travels through the particle and the light that travels a same physical distance through the surrounding medium [Snelling, 2007].

The comparison results of Farias et al. (1996) confirmed that

1. The light absorption by soot aggregates is insensitive to soot morphology. The effect of fractal dimension $\left(D_{\mathrm{f}}\right)$ on the performance of the RDG-PFA was generally found to be insignificant.

2. For soot aggregates characterized by $D_{\mathrm{f}} \approx 1.8$, the RDG-PFA approximation is in good agreement with the IEFS solution and the deviation from the IEFS is within $10 \%$ as long as the primary particles in aggregates are within the Rayleigh scattering limit, which means the primary particle size parameter, 
$x_{\mathrm{p}}$, is less than approximately 0.3 and the phase shift parameter is much less than unity.

For the current project, the primary particle diameter of soot aggregates generated from the inverted diffusion flame burner (described in detail in the Section 3.4.3) is $33.2 \mathrm{~nm}$ [Coderre et al., 2008]. The light wavelength used in the experiments is $577 \mathrm{~nm}$. The primary particle size parameter, $x_{\mathrm{p}}$ is thus expected to be,

$$
x_{\mathrm{P}}=\pi d_{\mathrm{P}} / \lambda=\pi \cdot 33.2 \mathrm{~nm} / 577 \mathrm{~nm}=0.181<0.3
$$

satisfying the size constraint and the phase shift becomes,

$$
\frac{2 \pi d_{\mathrm{p}}|m-1|}{\lambda}=0.362<1,
$$

which is acceptably below the limit outlined by Farias et al. (1996). Therefore it is concluded that the RDG-PFA theory applies to soot-containing plumes relevant to the current project.

A significant feature of RDG theory is that the absorption coefficient, $C_{\mathrm{a}}^{\mathrm{a}}$ for aggregates, is related to the absorption cross-section of the primary particles through the relationship:

$$
C_{\mathrm{a}}^{\mathrm{a}}=N_{\mathrm{p}} \cdot \alpha_{\mathrm{a}}^{\mathrm{p}}=N_{\mathrm{p}} \cdot \frac{\pi^{2} d_{\mathrm{p}}^{3} E(m)_{\lambda}}{\lambda},
$$

where $N_{\mathrm{p}}$, the particle number density, is the number of primary particles per unit volume (the unit of $N_{\mathrm{p}}$ is $\# / \mathrm{m}^{3}$ ). In other words, in RDG, the individual primary particles absorb light independently (i.e., as they would in the Rayleigh limit) and therefore the absorption of light by aggregates is proportional to the volume of the aggregates and is 
insensitive to soot morphology. Therefore, the light absorption by aggregates can be used to measure soot concentration.

\subsection{Two-Dimensional Line-Of-Sight Attenuation (2D-LOSA) Technique}

\subsubsection{Theoretical Derivation}

The technique studied in this thesis is based on the optical diagnostic two-dimensional Line-of-Sight Attenuation (2D-LOSA). LOSA is a commonly used non-intrusive optical diagnostic for the quantification of soot volume fraction in flames [Greenberg and $\mathrm{Ku}$, 1997; Snelling et al., 1999; Thomson et al., 2008a]. The key difference between LOSA and opacity measurements is that the LOSA technique is done with monochromatic light, which enables the use of theories to extract a quantitative measurement.

The basic method of attenuation measurements is that the intensity of light $\left(I_{\lambda_{0}}\right)$ entering an attenuating medium, such as a plume, and the light intensity after transmitting through the attenuating medium $\left(I_{\lambda}\right)$ are measured (see Figure 2.6). 


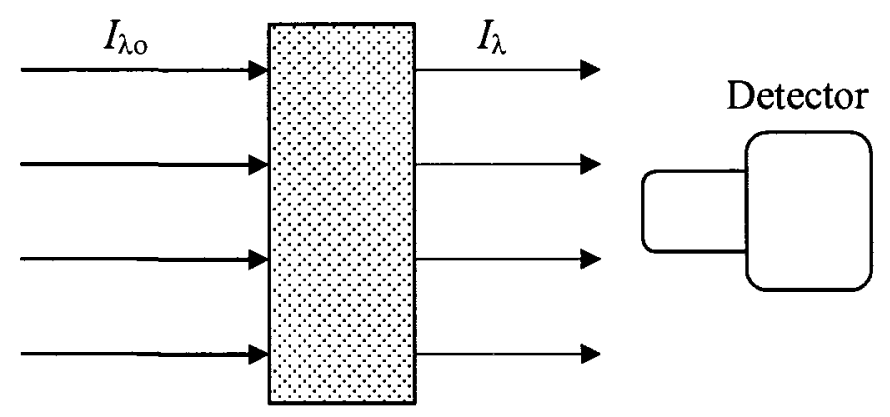

Air $\quad \begin{aligned} & \text { Attenuating Air } \\ & \text { medium }\end{aligned}$

Figure 2.6: Transmission can be determined from the detector response with and without attenuating medium between source and detector.

The attenuation of light by a medium is predicted by the well-known Beer-Lambert law [Greenberg and Ku; 1997, Siegell and Howell, 1981]. From the Beer-Lambert law, transmissivity is defined as the ratio of the transmitted intensity, $I_{\lambda}$, to the original beam intensity, $I_{\lambda \mathrm{o}}$, and is related to the extinction coefficient $\left(C_{\mathrm{e}}^{\lambda}\right)$ through:

$$
\tau_{\lambda}=\frac{I_{\lambda}}{I_{\lambda 0}}=\exp \left[-\int C_{\mathrm{e}}^{\lambda}(s) d s\right]
$$

Since the attenuation (or extinction) is the sum of scattering and absorption, the extinction coefficient $C_{\mathrm{e}}^{\lambda}$ is written as the sum of the absorption coefficient, $C_{\mathrm{a}}^{\lambda}$, and scattering coefficient, $C_{\mathrm{s}}^{\lambda}$, [Bohren and Huffman, 1983],

$$
C_{\mathrm{a}}^{\lambda}+C_{\mathrm{s}}^{\lambda}=C_{\mathrm{e}}^{\lambda} .
$$

Rearranging the equation above, we get

$$
C_{\mathrm{a}}^{\lambda}\left(1+\frac{C_{\mathrm{s}}^{\lambda}}{C_{\mathrm{a}}^{\lambda}}\right)=C_{\mathrm{e}}^{\lambda},
$$

or: 


$$
C_{\mathrm{a}}^{\lambda}\left(1+\rho_{\mathrm{sa}, \lambda}\right)=C_{\mathrm{e}}^{\lambda},
$$

where $\rho_{\mathrm{sa}, \lambda}$ is the ratio of scattering to absorption coefficient, $\rho_{\mathrm{sa}}=\frac{C_{\mathrm{s}}^{\lambda}}{C_{\mathrm{a}}^{\lambda}}$.

Substitution of Equation 2.10 into Equation 2.7, leads to

$$
\tau_{\lambda}=\frac{I_{\lambda}}{I_{\lambda_{0}}}=\exp \left[-\left(1+\rho_{\mathrm{sa}}\right) \int C_{\mathrm{a}}^{\lambda}(s) d s\right] .
$$

Taking the natural logarithm of both sides of the equation above yields:

$$
\ln \left(\tau_{\lambda}\right)=\ln \left(\frac{I_{\lambda}}{I_{\lambda 0}}\right)=-\left(1+\rho_{\mathrm{sa}}\right) \int C_{\mathrm{a}}^{\lambda}(s) d s .
$$

Although absorption usually dominates in a soot aerosol, scattering is not entirely absent. Historically, it has been common practice to assume that $\rho_{\mathrm{sa}}$ is equal to zero (i.e., that scatter is negligible). This assumption would be reasonable if primary particles were un-aggregated and in the Rayleigh limit. However, when soot is formed into aggregates, scattering can be significant [Musculus et al., 2005, Koylu and Feath, 1994] and can influence the interpretation of light attenuation measurements.

The value of $\rho_{\mathrm{sa}}$ depends on the soot morphology and refractive properties, and the wavelength of the light used in the attenuation measurements. As discussed in Section 2.2.3, RDG theory is well suited to describe light scatter by soot aggregated and can be used to assess the potential error that the collection of scattered light introduces into attenuation measurement. For example, Faeth and co-workers (1995), Koylu and Faeth (1994), Krishnan et al. (2001) determined that the total scattering to absorption ratio $\left(\rho_{\mathrm{sa}}\right)$ for soot aggregates investigated in the overfire region of acetylene, ethylene, and propane turbulent diffusion flames were approximately $0.41,0.29$, and 0.22 , respectively at $\lambda=514 \mathrm{~nm} . \quad \rho_{\mathrm{sa}}$ tends to increase with increasing propensity of fuel to soot. 
Available data show that the main component in flare gas is typically methane with decreasing amounts of higher hydrocarbons [Johnson et al., 2001]. Typically, methane has a lower sooting propensity than propane. Therefore, it is reasonable to assume that $\rho_{\mathrm{sa}}$ for soot produced from a methane turbulent diffusion flame will be below 0.2 [Johnson and Thomson, 2005].

Based on RDG-PFA theory, the soot volume fraction can be related to soot absorption coefficient, $C_{\mathrm{a}}^{\lambda}$, at a specific wavelength, $\lambda$, through the relationship:

$$
f_{\mathrm{v}}=\frac{C_{\mathrm{a}}^{\lambda} \cdot \lambda}{6 \pi E(m)_{\lambda}}
$$

where $E(m)_{\lambda}$ is the soot refractive index absorption function. The magnitude of $E(m)_{\lambda}$ has not been convincingly established in the literature, although researchers such as Lee and Tien (1980), Dalzell and Sarofim (1969), Dobbins (1994), Koylu and Faeth (1996), Wu et al. (1997), and Krishnan et al. (2000) have employed various means to estimate it. These works yield values of $E(m)_{\lambda}$ in the range of 0.21 to 0.42 . Canteenwalla (2007) summarized available data published in the literature and calculated the mean and standard error for $E(m)_{\lambda}$ of $0.32 \pm 0.01$ assuming $E(m)_{\lambda}$ to be independent of wavelength, which is a common assumption when using $E(m)_{\lambda}$ in the visible and near infrared (Krishnan et al., 2000). However, most recently, Thomson et al. (2008b) applied a spectrally resolved LOSA diagnostic (Spec-LOSA) to an ethylene/air flame in the wavelength range of 450 to $950 \mathrm{~nm}$ and observed that there is significant decrease of the absorption function with increasing wavelength. For the present investigation, at the wavelength of $577 \mathrm{~nm}$ in the current setup, a value of $E(m)_{\lambda}=0.26$ is assumed, which is reported by Dobbins et al. (1991) and used by Snelling et al. (1999). 


\subsubsection{Use of 2D-LOSA to Estimate Soot Mass Emission Rate}

The amount of soot emitted from a flare can be described as a mass flow rate of soot, $\dot{m}_{\text {soot }}$. By integrating across a cross-section of the plume perpendicular to the flow direction, the mass flow rate of soot is calculated as

$$
\dot{m}_{\mathrm{soot}}=u \rho_{\mathrm{soot}} \int_{A_{\mathrm{cs}}} f_{\mathrm{v}} d A,
$$

where $u$ is the plume velocity, $\rho_{\text {soot }}$ is soot density, $f_{\mathrm{v}}$ is the soot volume fraction, $A_{\mathrm{cs}}$ is the plume cross-sectional area, and the integral is performed over an area normal to the plume propagation direction. For simplicity, the velocity has been assumed to be uniform over the cross-section of the plume.

The value of soot density is still widely disputed. Amongst the many measurements of soot density, a soot density of $1.8-1.9 \mathrm{~g} / \mathrm{ml}$ is by far the most commonly cited value in the combustion community [e.g., Rossman and Smith, 1943; Flower and Bowman; 1986, Dobbins, et al., 1994; Wu, et al., 1997].

In evaluating the integral over the cross-section of the plume, we choose $x$ to represent the coordinate along the optical axis, $y$ to be perpendicular to $x$ in the crosssectional plane of the plume, and $z$ to be along the plume propagation direction as shown in Figure 2.7. After substituting Eq.2.13 into Equation 2.14 and breaking the area integral into an $x$ and $y$ component, the mass flow rate of soot is expressed by:

$$
\dot{m}_{\text {soot }}=\frac{u \rho_{\text {soot }} \lambda}{6 \pi E(m)_{\lambda}} \iint C_{\mathrm{a}}^{\lambda}(x, y) d x d y .
$$

Finally combining Equation 2.12 and Equation 2.15, yields

$$
\dot{m}_{\text {soot }}=\frac{-u \rho_{\text {soot }} \lambda}{6 \pi E(m)_{\lambda}\left(1+\rho_{\text {sa }}\right)} \int \ln \left(\tau_{\lambda}(y)\right) d y .
$$




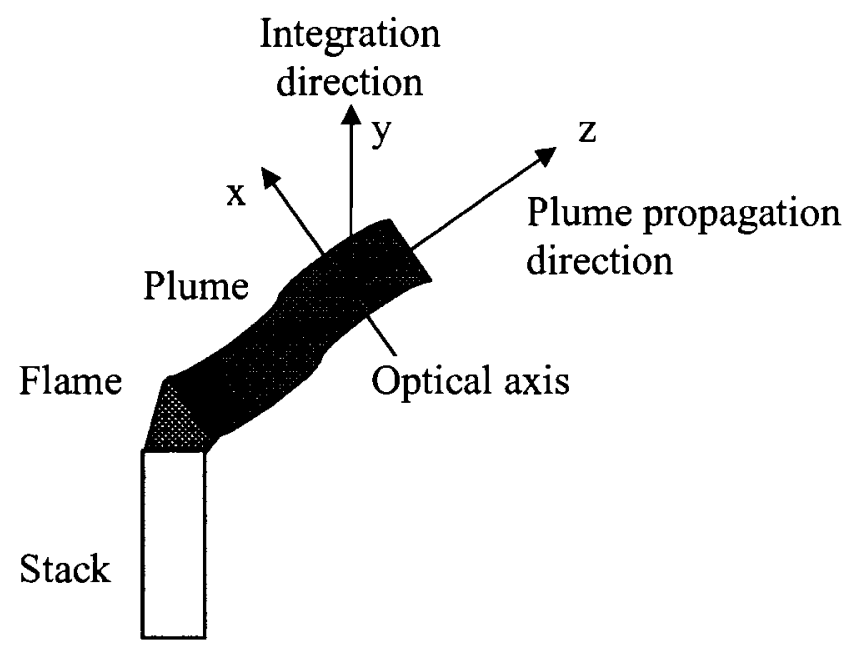

Figure 2.7: Definition of coordinate directions for a plume transmissivity measurement.

Thus, measurement of the plume transmissivity $\left(\tau_{\lambda}\right)$ using the 2D-LOSA technique can provide quantitative measurements of the soot mass emission rate of the flare.

During the evaluation of this method, it is convenient to normalize the integration of the natural logarithm of the local transmissivity by the width of the plume, defining the term $\kappa$ as follows:

$$
\kappa=-\frac{1}{w} \int \ln \left(\tau_{\lambda}(y)\right) d y
$$

Since the term $\kappa$ inherently considers transmissivity variation across the plume and is independent of plume width, this non-dimensional parameter can be used to compare results of experiments with different plume sizes and shapes. Therefore, results from labscale plumes presented in Chapter 5 can be applied to practical-scale plumes in the field. To relate $\kappa$ to the actual soot flux, $\dot{m}_{\text {soot }}$, Equation 2.18 can then be used, which includes a multiplication by the actual plume width: 


$$
\dot{m}_{\text {soot }}=\frac{u \rho_{\text {soot }} \lambda \cdot w}{6 \pi E(m)_{\lambda}\left(1+\rho_{\mathrm{sa}}\right)} \cdot \kappa
$$

In subsequent chapters, during the evaluation of the sky-LOSA technique, Equation 2.18 is typically used. However, in a field application, soot emission rate could be directly obtained from Equation 2.16.

\subsubsection{Theoretical Implementation}

\subsubsection{Three-image Method}

The plume transmissivity, $\tau_{\lambda}(y)$ from Eq. 2.18 , is measured using the 2D-LOSA optical diagnostic technique. In the original implementation of 2D-LOSA, four images are captured to measure the transmissivity in the lab [Snelling et al., 1999]. These images are called transmission, incandescence, background, and dark. The transmission image records the intensity of light from the source after it passes through the attenuating medium; the incandescence image records the intensity emitted by the attenuating medium (i.e., the incandescent emission from hot soot) with the light source blocked. The background image records the intensity of the light source in the absence of the attenuating medium; the dark image records the intensity with no attenuating medium and the light source blocked. In the current case where the soot source is not incandescing significantly (i.e., cool over-fired soot), dark and incandescence are equal and therefore only three images are needed to measure the transmissivity in the image field, in what is referred to here as a three-image method.

The measured light intensity without the attenuating source (i.e., $I_{\lambda o}$ ) is equal to background - dark and the measured light intensity with the attenuating source (i.e., $\left.I_{\lambda}\right)$ is equal to transmission - dark. The ratio of $I_{\lambda} / I_{\lambda_{0}}$ is equal to the soot transmissivity, $\tau$. 
Example images used to calculate transmissivity for a sample soot plume are shown in Figure 2.8 below.

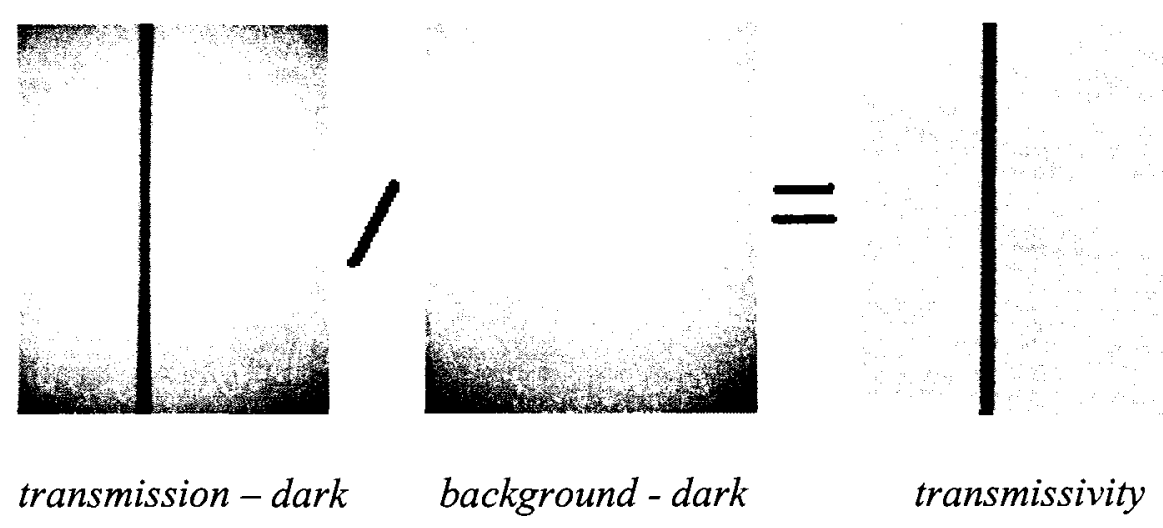

Figure 2.8: Example of three-image method. The black line in the figure is an actual plume generated from a lab controlled burner.

\subsubsection{Single-image Method}

In a field application of 2D-LOSA to measure plume transmissivity using sky radiation as the light source, it is impossible to isolate the background from the attenuating medium since neither the plume nor sky can be 'turned off.' Therefore it is desirable to measure plume transmissivity using a transmission image alone, in what is referred to here as a one-image method.

In the three-image method of 2D-LOSA, the background intensity is typically measured by obtaining an image of the light source intensity in the absence of the attenuating medium. However, if it is not possible to isolate the background from the attenuating medium, it becomes necessary to determine the background by different means. The premise of the present method is that the background in the attenuation region of the image (i.e., where the plume is present) can be estimated using information available from the transmission image in the regions outside of the attenuating material (e.g. outside of the plume). Specifically, if the background varies in a smooth and 
predictable manner, the background intensity in the region where the plume is present can be predicted through interpolation from regions of the image beyond the plume.

In the present experiments, plumes are typically centred left to right and appear vertical in the transmission images (see Figure 2.8). Since an integral of transmissivity is required across the cross-section of the attenuating medium perpendicular to the optical axis, the transmission image is divided into horizontal strips at various heights in the image. For each strip, the data to the left and to the right of the plume is used to create an interpolated synthetic background in the region of the plume. An analysis of the background interpolation procedure is presented in Section 4.1.1.

A second consideration with the one-image method is that the dark image cannot be obtained in a field application and must also be obtained by alternate means. It is useful to review the components that contribute to counts in a typical dark image. The first is fixed counts in the image which are an artifact of Analog-to-Digital (A/D) conversion and are a specific feature of the $\mathrm{CCD}$ and $\mathrm{A} / \mathrm{D}$ combination, which is invariant with time. The second is dark count accumulation which increases linearly with exposure time. These first two count sources can be quantified by acquiring an image with the system with the lens blocked from any input light (i.e., with the lens cover on) for the same exposure time used for the transmission measurement. A third count source in the dark image is spurious light (i.e., light not originating from the light source used in the measurements) which reaches the $\mathrm{CCD}$ and can contribute to the dark count reading. In the present system, where a camera lens is directly coupled to the CCD, spurious light should be strongly rejected and is assumed to be negligible. Therefore, the dark image is an image acquired by the system with the lens blocked. The validity of this assumption 
was tested by comparing dark images in the lab setting with and without the lens covered. It was found that spurious light contributed on average 4 counts $(\sim 0.01 \%)$ to the image.

\subsection{Scattering and Absorption of Light by Non-Soot Particles}

One assumption of the current diagnostic is that the light is only attenuated by soot as it passes through the plume. This will be true if soot particulates and gases are the only constituents of the plume; however, if any other non-soot particles (e.g., condensed water droplets and sulphate and/or nitrate particles) are present in the plume, they could interfere with the measurements. Potential influences of scattering by other particles are investigated in this section.

\subsubsection{Water Vapour Condensation}

There are significant variations in the composition of solution gases being flared at well sites. However, it is known that the primary component of solution gas is methane with decreasing amount of heavier gaseous hydrocarbons (i.e., ethane $\left(\mathrm{C}_{2} \mathrm{H}_{6}\right)$, propane $\left(\mathrm{C}_{3} \mathrm{H}_{8}\right)$, butane $\left(\mathrm{C}_{4} \mathrm{H}_{10}\right)$, etc.), varying amounts of hydrogen sulphide $\left(\mathrm{H}_{2} \mathrm{~S}\right)$, and small amounts of carbon dioxide and nitrogen [Johnson et al., 2001]. Since natural gas has a high hydrogen-to-carbon ratio, water vapour in the combustion products is high relative to other fossil fuels. The possibility of water vapour condensing to form water droplets which will interfere with soot transmissivity measurements is therefore a concern.

The probability that water vapour in a plume will condense is dependent on the plume temperature, water saturation pressure at this temperature, partial pressure of water vapour in the combustion products, and water vapour pressure (or relative humidity) of the ambient air that is entrained into the plume. When the combustion products leave the flame, they are significantly hotter than the surrounding atmosphere and the water vapour 
partial pressure is relatively high. Due to dilution with ambient air entrained into the plume as the plume evolves, the temperature of the plume drops and water vapour partial pressure decreases. A lower plume temperature reduces the water vapour saturation pressure and promotes condensation while dilution also decreases water vapour pressure since the entrained air is significantly drier than combustion products, thus suppressing condensation. The balance between these opposing factors is critical in determining whether condensation will take place.

To determine whether vapour condensation will occur, the plume temperature and volume fraction of entrainment air must be known. Poudenx (2000) studied plumes of diffusion flames within a controlled wind tunnel. He measured and established concentration contours for relevant species and mean temperature maps for different cross-sections of plumes. Since we are primarily interested in the near-field of plumes, in the vicinity of the emission source, we chose $\left[\mathrm{O}_{2}\right]\left(\mathrm{O}_{2}\right.$ concentration $)$ contours of a crosssection $110 \mathrm{~cm}$ downstream of the stack, shown in Figure 2.9 (adapted from Poudenx, 2000). 


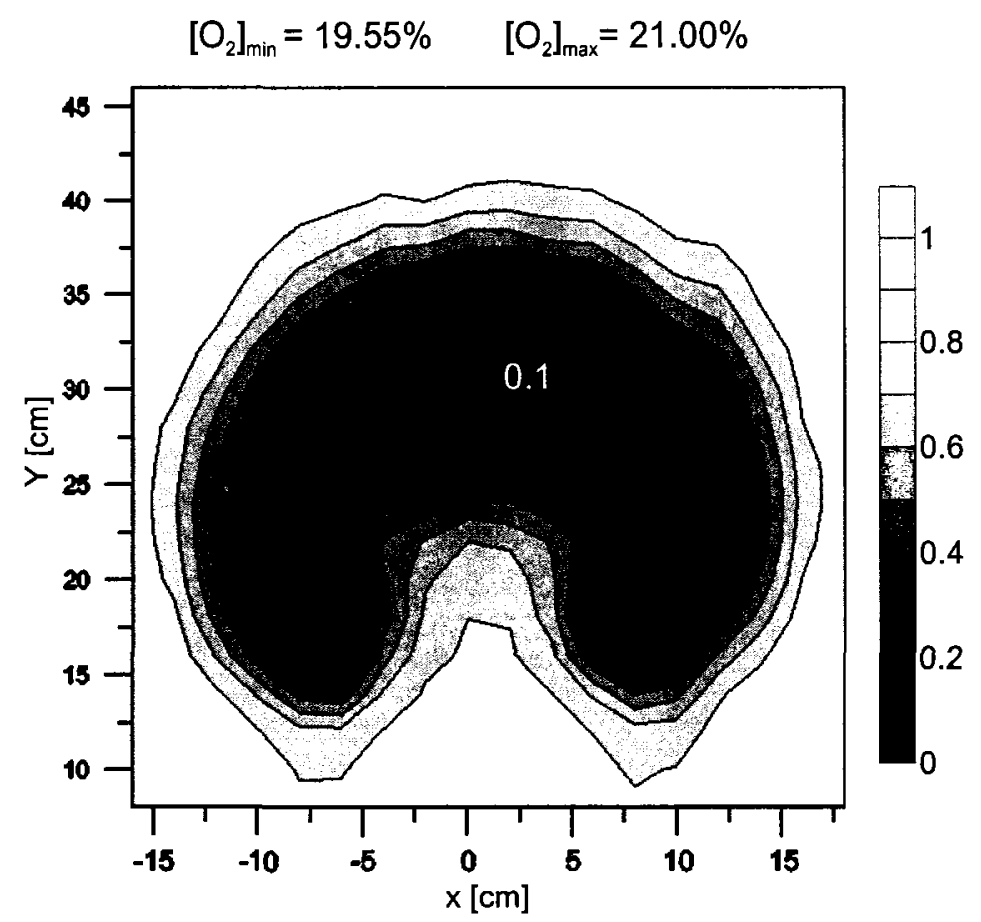

Figure 2.9: Normalized $\mathrm{O}_{2}$ profile at the location of $110 \mathrm{~cm}$ downstream of the stack of a lab-scale flare in a wind tunnel. Crosswind velocity $2 \mathrm{~m} / \mathrm{s}$, plume exit velocity 1 $\mathrm{m} / \mathrm{s}$, and stack diameter $2.21 \mathrm{~cm}$ [Poudenx, 2000].

If we assume that the combustion reaction takes place along a stoichiometric contour, then there will be no oxygen in the combustion products. The products then propagate away from the flame while mixing with surrounding air. The degree of dilution can be quantified based on the oxygen concentration in the plume. A high level of oxygen would correspond to a high degree of dilution, and a low level of oxygen to a low level of dilution in the sample. We can determine the amount of air entrained in the plume from the oxygen concentration in a sample from the following equation [Poudenx, 2000].

$$
\left[\mathrm{O}_{2}\right]_{\text {sample }}=\left[\mathrm{O}_{2}\right]_{\text {entrain }}(1-\alpha)
$$

where $\left[\mathrm{O}_{2}\right]_{\text {sample }}$ is the $\mathrm{O}_{2} \mathrm{~mol}$ fraction of a sample, 
$\left[\mathrm{O}_{2}\right]_{\text {entrain }}$ is the $\mathrm{O}_{2}$ mol fraction of the entrainment air which is approximately $21 \%$, and

$\alpha$ is volume fraction of combustion products in the sample.

From Figure 2.9, we can see for a location $110 \mathrm{~cm}$ downstream of the stack, $\left[O_{2}\right]_{\text {sample }}$ is lowest in the centre of the plume at $19.55 \%$ and is highest in the edge of the plume at around $21 \%$. Assuming $\left[\mathrm{O}_{2}\right]$ changes linearly from the centre to the edge of the plume, $\left[\mathrm{O}_{2}\right]_{\text {sample }}$ is calculated along the width of the plume and is shown in the $2^{\text {nd }}$ column of Table 2.1. The volume fractions of entrained air $(1-\alpha)$ and combustion products $(\alpha)$ are calculated based on Equation 2.19 at this cross-section and the results are also shown in Table 2.1.

Table 2.1: Estimated volume fraction of entrained air and products at a plume cross-section $110 \mathrm{~cm}$ downstream of the stack

\begin{tabular}{|c|c|c|c|}
\hline $\begin{array}{c}\text { Distance from the centre to } \\
\text { the edge of the plume }(\mathbf{c m})\end{array}$ & {$\left[O_{2}\right]_{\text {sample }}$} & $\begin{array}{c}\text { Volume Fraction } \\
\text { of Products } \\
(\alpha)\end{array}$ & $\begin{array}{c}\text { Volume Fraction of } \\
\text { Entrained Air } \\
(1-\alpha)\end{array}$ \\
\hline 0 & 19.55 & 0.069 & 0.931 \\
\hline 5 & 19.98 & 0.049 & 0.951 \\
\hline 10 & 20.41 & 0.028 & 0.972 \\
\hline 15 & 20.84 & 0.008 & 0.992 \\
\hline
\end{tabular}

The water vapour in the plume comes from both entrained air and combustion products. Calculations of the total water vapour in the plume are performed in two ways. As a first approximation, it is assumed that there is no radiative heat loss from the plume. The temperature of combustion products is at the adiabatic flame temperature for a methane-fueled flare with reactants at ambient temperature. As an improved estimate, a range of radiative heat loss fractions are included in the determination of the combustion 
product temperature. In all calculations, the local plume temperature is calculated from the heat balance between the ambient temperature and the combustion products temperature. The assumptions are:

- Ambient air temperature, $T_{\text {air }}$, ranges from $-30^{\circ} \mathrm{C}$ to $30^{\circ} \mathrm{C}$,

- Ambient air is saturated (100\% relative humidity), which represents a conservative worst-case scenario at any temperature, and

- Ambient pressure, $\mathrm{P}_{\infty}$, of $101.325 \mathrm{kPa}$.

The partial pressures of water vapour in ambient air $\left(P_{w, \text { air }}\right)$ at different ambient temperatures are obtained from a saturated solid-vapour table and saturated steam: temperature table [Sonntag and Van Wylen, 1985]. The partial pressure of water vapour in the combustion products $\left(P_{w, \text { comb }}\right)$ is found by assuming stoichiometric combustion of methane according to Equation 2.20:

$$
\mathrm{CH}_{4}+2\left(\mathrm{O}_{2}+3.76 \mathrm{~N}_{2}\right) \rightarrow \mathrm{CO}_{2}+2 \mathrm{H}_{2} \mathrm{O}+7.52 \mathrm{~N}_{2} .
$$

Since methane has the highest hydrogen-to-carbon ratio of any hydrocarbon fuel, this assumption is quite conservative and would overestimate the actual amount of water in the combustion products. Assuming complete combustion with negligible dissociation:

$$
P_{w, \text { comb }}=\left(\frac{2}{1+2+7.52}\right) \cdot P_{\infty}=19.25 \mathrm{kP}_{\mathrm{a}} \text {. }
$$

The total partial pressure of water vapour in the plume from both combustion products and entrained air, $P_{\text {total }}$, can be calculated as:

$$
P_{\text {total }}=(1-\alpha) \cdot P_{w, \text { air }}+\alpha \cdot P_{w, \text { comb }} \text {. }
$$

Total partial pressures of water vapour in the plume are then compared with water saturation pressures, $P_{\text {sat }}$, at the plume temperature for different ambient air temperatures. 
If $P_{\text {total }}$ reaches $P_{\text {sat }}$ at its corresponding plume temperature, water will begin to condense. Since these calculations are strongly dependent on the ambient temperature, plume temperatures must be determined for different ambient temperatures. The plume temperatures are estimated initially based on the following assumptions:

- combustion products and entrained air are blended by perfect mixing; and - radiation and conduction heat transfer from the products are negligible.

From Table 2.1, columns 3 and 4, we know the volume fractions of the entrained air and products at different radial positions of the cross-section of the plume. After blending a certain amount of entrained air with its associated heat capacity and a certain amount of products with different heat capacities, the plume reaches its energy equilibrium. The energy balance is thus:

$$
\begin{aligned}
& (1-\alpha) \cdot \int_{T_{\mathrm{air}}}^{T_{\mathrm{plume}}}\left(a \cdot C_{\mathrm{p}},\right. \text { Dryair } \\
& \alpha \cdot \int_{T_{\mathrm{plume}}}^{T}\left(d \cdot C_{\mathrm{p}, \mathrm{CO} 2}(T)+e \cdot C_{\mathrm{p}, w, \text { air }}(T)\right) d T=
\end{aligned}
$$

where $C_{\mathrm{p}} \mathrm{s}$ are specific heats at constant pressure for dry air, water vapour from ambient air, $\mathrm{CO}_{2}$, water vapour from combustion, and $\mathrm{N}_{2}$. Values of $C_{\mathrm{p}}$ are obtained from the table of curve-fit coefficients for thermodynamic properties [Turns, 1996]. The initial temperature of the combustion products, $T$, in the absence of heat loss is assumed to be equal to the adiabatic flame temperature for a methane-fueled flare with reactants at ambient temperature. $T_{\text {plume }}$ is the final plume temperature. The coefficients $a, b, d, e$, and $f$ are the molar fractions of each species.

For example, when assuming the ambient air is saturated at $-30^{\circ} \mathrm{C}$, the water vapour partial pressure, $P_{w}$, air, is $0.0381 \mathrm{kPa}$ [Sonntag and Van Wylen, 1985]. Therefore, 


$$
\begin{gathered}
a=\frac{P_{w, \text { air }}}{P_{\infty}}=\frac{0.0381 \mathrm{kPa}}{101.3 \mathrm{kPa}}=3.76 \times 10^{-4}, \text { and } \\
b=1-\frac{P_{w, \text { air }}}{P_{\infty}}=0.9996 .
\end{gathered}
$$

For the products, from Equation 2.20:

$$
\begin{gathered}
d=\frac{1}{1+2+7.52}=0.0950, \\
e=\frac{2}{1+2+7.52}=0.190, \text { and } \\
f=\frac{7.52}{1+2+7.52}=0.715 .
\end{gathered}
$$

To verify the validity of the assumption of perfect mixing without heat transfer, $T_{\text {plume }}$ is calculated at the centre and edge of the plume at Poudenx's sampling conditions $\left(20^{\circ} \mathrm{C}, 96 \mathrm{kPa}\right)$ and is compared with the results from plume temperature measurements of Poudenx (2000).

As seen in Table 2.2, the simulation result of $T_{\text {plume }}$ at the centre of the plume has a discrepancy with the measured data but the result at the edge of the plume agrees well with the measured data. The result suggests that besides the heat transfer through the course of the mixing, a heat loss due to plume radiation has to be considered in the energy balance. Since the temperature at the centre is higher than that at the edge of the plume, it is expected that the heat lost due to radiation would be more significant at the centre than that at the edge of the plume. 
Table 2.2: Comparison of estimated $T_{\text {plume }}$ with measured data

\begin{tabular}{|c|c|c|}
\hline Evaluation Position & $\begin{array}{c}\text { Simulation } \\
\text { Results }\end{array}$ & $\begin{array}{c}\text { Measurement Results from Poudenx } \\
(2000)\end{array}$ \\
\hline $\begin{array}{c}\text { At the centre of the } \\
\text { plume }\end{array}$ & $189^{\circ} \mathrm{C}$ & $148^{\circ} \mathrm{C}$ \\
\hline At the edge of the plume & $39^{\circ} \mathrm{C}$ & $40^{\circ} \mathrm{C}$ \\
\hline
\end{tabular}

Heat loss from flares is dominated by radiative loss, which is expressed as the total radiant power emitted from a flare as a fraction of the total rate of heat release [Cook $e t$ al., 1987]. Guigard and Kindzierski (2000) summarized values of the fraction of heat radiated given in the literature. Selected data for methane flames are listed in Table 2.3

Table 2.3: Selected values for fraction of heat radiated from methane flames given in the literature*

\begin{tabular}{|c|c|c|c|c|}
\hline \multicolumn{2}{|l|}{ Citation } & $\begin{array}{c}\text { Fraction of } \\
\text { heat }\end{array}$ & \multicolumn{2}{|l|}{ Notes } \\
\hline Tan & 1967 & 0.2 & Methane & $\begin{array}{l}30 \text { in jet } \\
\text { diameter }\end{array}$ \\
\hline API RP 521 & 1969 & 0.16 & Methane, in still air & $\begin{array}{c}1.9 \mathrm{~cm} \text { burner } \\
\text { diameter }\end{array}$ \\
\hline API RP 521 & 1969 & 0.20 & Natural Gas $\left(95 \% \mathrm{CH}_{4}\right)$ & $\begin{array}{c}20.3 \mathrm{~cm} \text { burner } \\
\text { diameter }\end{array}$ \\
\hline Brzustowski et al. & 1975 & 0.155 & $\begin{array}{c}\text { Methane, gas exit } \\
\text { velocity of } 30.9 \mathrm{~m} / \mathrm{s} \text { in } \\
\text { still air } \\
\end{array}$ & \multirow{4}{*}{$\begin{array}{l}5 \mathrm{~mm} \text { jet } \\
\text { diameter }\end{array}$} \\
\hline Brzustowski et al. & 1975 & 0.17 & $\begin{array}{c}\text { Methane, gas exit } \\
\text { velocity of } 24.5 \mathrm{~m} / \mathrm{s} \text { in } \\
\text { still air } \\
\end{array}$ & \\
\hline Brzustowski et al. & 1975 & 0.23 & $\begin{array}{l}\text { Methane, gas exit } \\
\text { velocity of } 30.9 \mathrm{~m} / \mathrm{s} \text { with } \\
\text { cross-wind } 2 \mathrm{~m} / \mathrm{s}\end{array}$ & \\
\hline Brzustowski et al. & 1975 & 0.26 & $\begin{array}{l}\text { Methane, gas exit } \\
\text { velocity of } 24.5 \mathrm{~m} / \mathrm{s} \text { with } \\
\text { cross-wind } 2 \mathrm{~m} / \mathrm{s}\end{array}$ & \\
\hline
\end{tabular}

*Adapted from Guigard and Kindzierski, (2000) 
From Table 2.3, we can see the values of fraction of heat radiated vary from 0.16 to 0.26 under different circumstances. Using 0.16 and 0.26 as the two bounding cases, plume temperatures are recalculated but with consideration for the radiative heat loss as shown schematically in Figure 2.10.

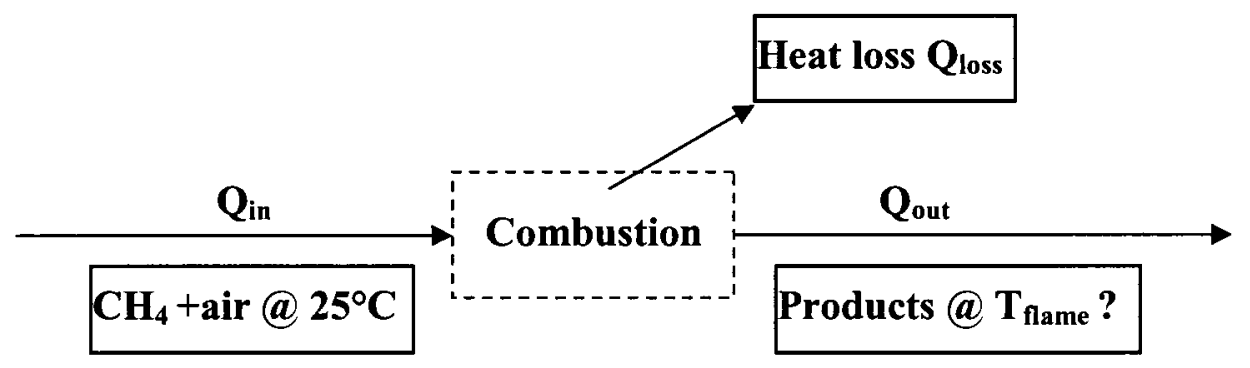

Figure 2.10: Schematic diagram of a control volume

Figure 2.10 shows a schematic diagram of a control volume that includes heat transfer and reactant temperature. Taking the combustion process as our control volume and applying the first law, we have

or

$$
\begin{gathered}
Q_{\text {in }}=Q_{\text {out }}+Q_{\text {loss }} \\
\left.N \cdot \bar{h}\right|_{\mathrm{CH}_{4}}+\left.N \cdot \bar{h}\right|_{\mathrm{O}_{2}}+\left.N \cdot \bar{h}\right|_{\mathrm{N}_{2}}=\left.N \cdot \bar{h}\right|_{\mathrm{CO}_{2}}+\left.N \cdot \bar{h}\right|_{\mathrm{H}_{2} \mathrm{O}}+\left.N \cdot \bar{h}\right|_{\mathrm{N}_{2}}+Q_{\text {loss }}
\end{gathered}
$$

where $N$ is the number of moles of each species in the reactants and products (as shown in Equation 2.20) and $\bar{h}$ is the specific molar absolute enthalpy for each species (absolute enthalpy per mole of fuel).

Substituting $\bar{h}$ with $\left(\bar{h}_{f}^{0}+\Delta \bar{h}\right)$, Equation 2.24 becomes :

$$
\begin{aligned}
& \underbrace{\left.N_{\mathrm{CH}_{4}} \cdot\left(\bar{h}_{f}^{0}+\Delta \bar{h}\right)\right|_{\mathrm{CH}_{4}}+\left.N_{\mathrm{O}_{2}} \cdot\left(\bar{h}_{f}^{0}+\Delta \bar{h}\right)\right|_{\mathrm{O}_{2}}+\left.N_{\mathrm{N}_{2}} \cdot\left(\bar{h}_{f}^{0}+\Delta \bar{h}\right)\right|_{\mathrm{N}_{2}}}_{\text {reactants at ambient temperature }} \\
& =\underbrace{\left.N_{\mathrm{CO}_{2}} \cdot\left(\bar{h}_{f}^{0}+\Delta \bar{h}\right)\right|_{\mathrm{CO}_{2}}+\left.N_{\mathrm{H}_{2} \mathrm{O}} \cdot\left(\bar{h}_{f}^{0}+\Delta \bar{h}\right)\right|_{\mathrm{H}_{2} \mathrm{O}}+\left.N_{\mathrm{N}_{2}} \cdot\left(\bar{h}_{f}^{0}+\Delta \bar{h}\right)\right|_{\mathrm{N}_{2}}}_{\text {at } T_{\text {llame }} \mathrm{K}}+Q_{\text {loss }}
\end{aligned}
$$


where $\bar{h}_{f}^{0}$ is the enthalpy of formation and $\Delta \bar{h}$ is the sensible enthalpy change (enthalpy change associated with temperature alone). $Q_{\text {loss }}$ is calculated as the fraction of heat loss multiplied the lower heating value of $\mathrm{CH}_{4}$. Using Equation $2.25, T_{\text {flame }}$ is solved by iteration.

To verify the plume temperature calculation, the simulation model is used at the centre and edge of the plume at the Poudenx sampling condition $\left(20^{\circ} \mathrm{C}, 96 \mathrm{kPa}\right)$. The results of calculations with and without radiative heat loss are listed in Table 2.4.

Table 2.4: Compare simulation results of plume temperatures with measured data

\begin{tabular}{|c|c|c|c|c|}
\hline \multirow{2}{*}{$\begin{array}{c}\text { Evaluation } \\
\text { Position } \\
\text { in the plume }\end{array}$} & $\begin{array}{c}\text { Measured data } \\
\text { from Poudenx } \\
(2000)\end{array}$ & $\begin{array}{c}\text { No heat } \\
\text { loss }\end{array}$ & $\begin{array}{c}\text { Heat loss } \\
\text { fraction of } \\
0.16\end{array}$ & $\begin{array}{c}\text { Heat loss } \\
\text { fraction of } \\
0.26\end{array}$ \\
\hline Centre & $148^{\circ} \mathrm{C}$ & $189^{\circ} \mathrm{C}$ & $152^{\circ} \mathrm{C}$ & $135^{\circ} \mathrm{C}$ \\
\hline Edge & $40^{\circ} \mathrm{C}$ & $39^{\circ} \mathrm{C}$ & $36^{\circ} \mathrm{C}$ & $33^{\circ} \mathrm{C}$ \\
\hline
\end{tabular}

It is observed that a better agreement is achieved at the centre of the plume for the high heat loss case, while no heat loss gives better results at the edge of the plume. Although the comparison is based only on one set of measurement data, this suggests that rapid heat loss by mixing likely dominates over radiation heat loss at the edge of the plume. As shown in Table 2.4, it is observed that the heat loss of 0.16 may be closer to the actual fraction for Poudenx' flame, however a heat loss of 0.26 is also used in the simulation as a conservative limit.

Substituting $T$ with $T_{\text {flame }}$ (for no heat loss and for heat loss with assumed radiative fractions of 0.16 and 0.26 ) into the upper limit of the integral in the right hand side of Equation 2.23 and solving for $T_{\text {plume, }}$, we obtained values of $T_{\text {plume }}$ and $P_{\text {sat }}$ for different ambient temperatures as presented in Table 2.5 and also shown graphically in Figure 2.11 . 
Table 2.5: The estimation of $T_{\text {plume }}, P_{\text {total }}$, and $P_{\text {sat }}$ at the edge of the plume

\begin{tabular}{|c|c|c|c|c|c|c|c|}
\hline \multirow[t]{2}{*}{$\begin{array}{l}T_{\text {air }} \\
\left({ }^{\circ} \mathrm{C}\right)\end{array}$} & \multicolumn{2}{|c|}{$\begin{array}{l}\text { Combustion } \\
\text { products \& air } \\
\text { perfect mixing } \\
\text { with no heat } \\
\text { loss }\end{array}$} & \multicolumn{2}{|c|}{$\begin{array}{c}\text { Combustion products } \& \text { air } \\
\text { perfect mixing assuming } \\
\text { fraction of heat radiated = } \\
0.16\end{array}$} & \multicolumn{2}{|c|}{$\begin{array}{c}\text { Combustion products \& air } \\
\text { perfect mixing assuming } \\
\text { fraction of heat radiated = } \\
0.26\end{array}$} & \multirow[t]{2}{*}{$\begin{array}{l}P_{\text {Total }} \\
(\mathrm{kPa})\end{array}$} \\
\hline & $\begin{array}{l}T_{\text {plume }} \\
\left({ }^{\circ} \mathrm{C}\right)\end{array}$ & $\begin{array}{c}P_{\text {sat. }} \\
(\mathbf{k P a})\end{array}$ & $\begin{array}{c}T_{\text {plume }} \\
\left({ }^{\circ} \mathrm{C}\right)\end{array}$ & $\begin{array}{c}P_{\text {sat. }} \\
(\mathbf{k P a})\end{array}$ & $\begin{array}{c}T_{\text {plume }} \\
\left({ }^{\circ} \mathrm{C}\right)\end{array}$ & $\begin{array}{c}P_{\text {sat. }} \\
(\mathbf{k P a})\end{array}$ & \\
\hline-30 & -11 & 0.239 & -14 & 0.182 & -16 & 0.151 & 0.192 \\
\hline-20 & -1 & 0.564 & -4 & 0.437 & -6 & 0.369 & 0.257 \\
\hline-10 & 9 & 1.16 & 5 & 0.872 & 3 & 0.767 & 0.412 \\
\hline 0 & 19 & 2.21 & 15 & 1.71 & 13 & 1.51 & 0.760 \\
\hline 10 & 29 & 4.25 & 25 & 3.17 & 23 & 2.84 & 1.37 \\
\hline 20 & 39 & 7.38 & 35 & 5.63 & 33 & 5.07 & 2.47 \\
\hline 30 & 49 & 12.3 & 45 & 9.59 & 43 & 8.71 & 4.37 \\
\hline
\end{tabular}

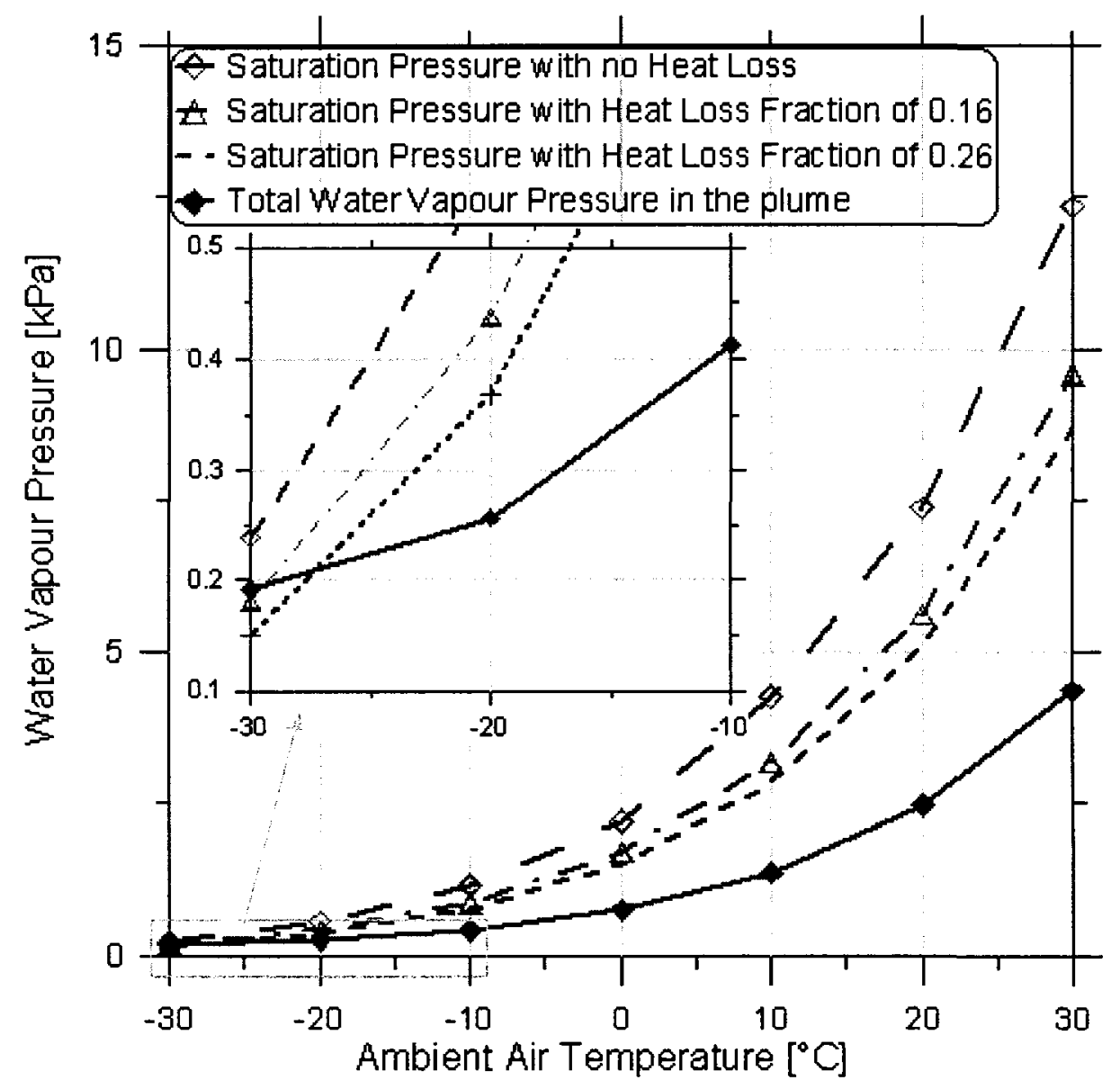

Figure 2.11 Total water vapour pressure of the plume and the saturation pressure at the plume edge temperature as a function of ambient air temperatures assuming ambient air saturated. A zoom-in figure between $-30^{\circ} \mathrm{C}$ to $-10^{\circ} \mathrm{C}$ is inserted to the original figure. 
As shown in Figure 2.11, the assumption of no heat loss from the plume leads to the highest $P_{\text {sat }}$ line (in olive) because the plume temperatures are highest. It is observed that there will be no water condensation happening within the full range of assumed ambient temperatures for the no heat loss scenario. Once heat loss from the plume is included, the plume temperatures drops, which leads to a drop of the $P_{\text {sat }}$ line. When the $P_{\text {sat }}$ line falls below the $P_{\text {total }}$ line, water condensation occurs. At the heat loss fraction value of 0.26 (the most conservative scenario), the $P_{\text {sat }}$ line (in moss green) crosses over with the $P_{\text {total }}$ line at around $-27^{\circ} \mathrm{C}$, which means no water condensation will occur when the ambient temperatures is above $-27^{\circ} \mathrm{C}$ at the edge of the plume.

To take into account the radial variation of both temperature and water vapour partial pressure in the plume, $P_{\text {sat }}$ and $P_{\text {total }}$ are calculated as a function of the distance from the centre to the edge of the plume cross section for the most conservative scenario of ambient temperature of $-30^{\circ} \mathrm{C}$ with relative humidity of $100 \%$ and the heat loss fraction of 0.26 . The simulation results are shown in Figure 2.12. 


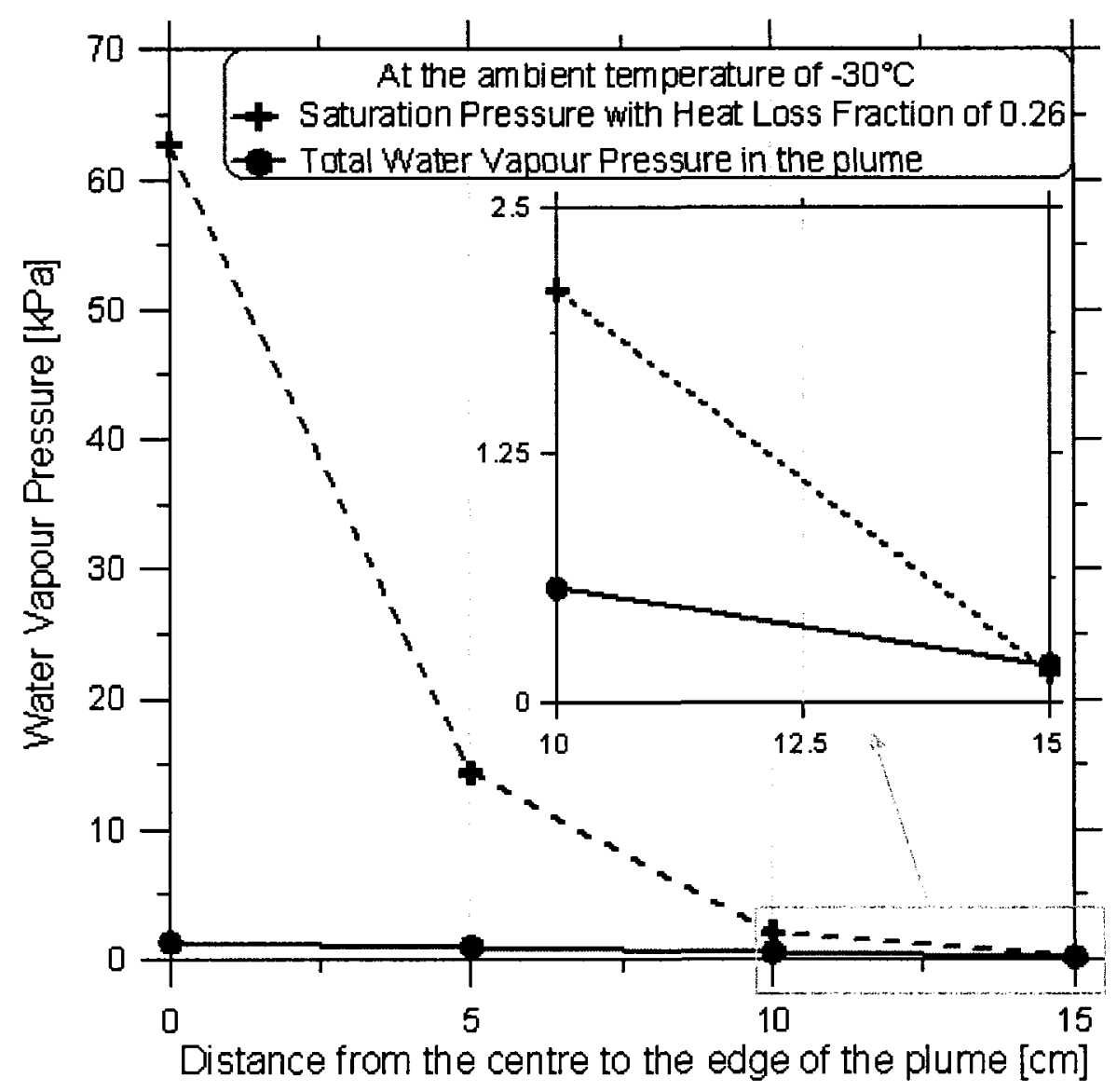

Figure 2.12: Total water vapour pressure and saturation pressure of the plume as a function of distance from the centre to the edge of the plume at ambient temperature of $-30^{\circ} \mathrm{C}$ with relative humidity of $100 \%$. A zoom-in figure between 10 $\mathrm{cm}$ to $15 \mathrm{~cm}$ is superimposed on the original figure.

Figure 2.12 shows that the radial position where the water condensation will occur is very close to the edge of the plume. Therefore, the results of Figure 2.11 represent the point of onset of condensation for the entire plume cross section.

When ambient temperatures are near or below $-27^{\circ} \mathrm{C}$, the possibility of water condensation should be evaluated on a case-by-case basis. The reasons are as follows. First, the heat loss fraction used in the current simulation is chosen based on high gas exit velocities (refer to Table 2.3). Secondly, the relative humidity in ambient air is assumed 
as $100 \%$. If conditions of a plume and ambient air are not close to those assumed in the present simulation, there could be a variation in the saturation temperature.

Overall, the present analysis suggests that for plumes of flares under common measurement conditions (i.e., temperatures above $-27^{\circ} \mathrm{C}$ and relative humidity of ambient air less than $100 \%$ ), water condensation will be insignificant and should not be a concern for plume transmissivity measurements. It should be noted that the presence of $\mathrm{SO}_{2}$ in a plume can lead to production of $\mathrm{H}_{2} \mathrm{SO}_{4}$, which can significantly raise the dew point [De Nevers, 2000]. This would affect the previous water condensation calculation. However, $\mathrm{SO}_{2}$ would only be formed in flares with significant amounts of hydrogen sulphide $\left(\mathrm{H}_{2} \mathrm{~S}\right)$, which represents approximately one-third or fewer of solution gas flares in Alberta [Johnson et al., 2001]. For flares with significant amounts of $\mathrm{H}_{2} \mathrm{~S}$, this analysis may need to be reexamined on a case by case basis.

\subsubsection{Sulfate and Nitrate Formation}

For flares in the upstream oil and gas industry, secondary particulate emissions may also be relevant in addition to primary particulate emissions of carbonaceous particles (i.e. soot). Secondary particulates are initially formed as gaseous pollutants which later form particles in the atmosphere. Two relevant classes of secondary particles or droplets are sulphates $\left(\mathrm{SO}_{4}\right)$ and nitrates $\left(\mathrm{NO}_{3}\right)$, which originate from sulphur and nitrogen oxides emitted as combustion products. Secondary particle formation takes place as a consequence of different physical and chemical processes. In general, the formation process is very complex and generally involves homogeneous nucleation (gas-phase oxidation) as well as heterogeneous nucleation (aqueous phase oxidation) [Querol et al., 1996]. Gas phase oxidation is currently believed to occur primarily by the reaction with 
free hydroxyl (OH) radical [Gillani et al., 1981; Hewitt, 2001; Lazaridis et al., 2001]. Aqueous phase oxidation is vapour condensation onto fine particles and is believed to be appreciable on the wet aerosol surfaces close to the point of emission [Cheng et al., 1987]. These processes determine the formation rate and the particle size.

Gillani and Wilson (1980) concluded that sunlight, plume dilution, background reactivity, and photochemical processes are the principal factors influencing secondary particle formation in a plume. Some field studies shown below have been carried out with the aim of estimating sulfate and nitrate formation rate in coal- or oil-fired power station plumes.

Summer and wintertime oxidation rates of $\mathrm{SO}_{2}$ were obtained in the plume from a northern Alberta power station by Lusis et al. (1978). In February the oxidation rate was found to be slow (less than $0.5 \% \mathrm{~h}^{-1}$ ), increasing to $1-3 \% \mathrm{~h}^{-1}$ in June. Forrest et al. (1981) found a daytime average conversion rate of $3 \% \mathrm{~h}^{-1}$ in summer, varying with time of day, and average of $0.5 \% \mathrm{~h}^{-1}$ at night. Another wintertime study for a power station plume in Georgia (Liebsch and De Pen, 1982) found that the highest conversion rate was $0.2 \% \mathrm{~h}^{-1}$, except when high relative humidity was observed. Under high humidity, the conversion rate increased to $2.3 \% \mathrm{~h}^{-1}$. Anlauf et al. (1982) found an average summertime $\mathrm{SO}_{2}$ oxidation rate of $4 \% \mathrm{~h}^{-1}$ in the plume of the Ontario Nanticoke coal-fired power station at downstream of the plume for ambient relative humidities of $30-50 \%$.

Gillani et al. (1981) studied the gas-to-particle conversion rate of sulphur emitted from a coal-fired power station in Kansas. They found the average sulphur formation rate was less than $3 \% \mathrm{~h}^{-1}$ and only occurred during the daytime. At the plume edge where plume dilution is greatest, conversion rates may be significantly enhanced. Zak (1981) 
observed daytime conversion rates up to $5.5 \% \mathrm{~h}^{-1}$ in a plume edge. Gillani et al. (1981) found $7.5 \% \mathrm{~h}^{-1}$ in plume edges. Mamane and Pueschel (1980) estimated sulphate formation rates in the plume of the Four Corners power station to be about $0.15 \% \mathrm{~h}^{-1}$ in the first $0.3 \mathrm{~h}$, increasing to $0.5 \% \mathrm{~h}^{-1}$ after two hours of transport in the atmosphere.

Heterogeneous reaction is expected to be most prevalent just after exhaust leaves the stack, before entrainment significantly dilutes the vapour concentration and lowers the saturation ratio [Mueller and Imhoff, 1994a]. Cheng et al. (1987) studied the plume from an oil sand extraction plant in Alberta. The measurements were taken $3.1 \mathrm{~km}$ downwind from the stack and the conversion rate of sulphate was $\sim 2.8 \% \mathrm{~h}^{-1}$ in winter and $\sim 6 \% \mathrm{~h}^{-1}$ in summer. The measurements also revealed an increase in the transformation rate of $\mathrm{SO}_{2}$ to particulate sulfate with plume travel time.

Hewitt (2000) reviewed $\mathrm{SO}_{2}$ and $\mathrm{NO}_{\mathrm{x}}$ chemistry and concluded $\mathrm{SO}_{2}$ and $\mathrm{NO}_{\mathrm{x}}$ compete for the same oxidizing radicals $(\mathrm{OH})$, and oxidation of $\mathrm{NO}_{2}$ is roughly 10 times faster than that of $\mathrm{SO}_{2}$. Hence the presence of $\mathrm{NO}_{\mathrm{x}}$ in a plume will inhibit $\mathrm{SO}_{2}$ oxidation rates (and sulphate formation rates). Hewitt summarized that in sunny conditions maximum $\mathrm{SO}_{2}$ and $\mathrm{NO}_{\mathrm{x}}$ conversion rates are $3 \% \mathrm{~h}^{-1}$ and $30 \% \mathrm{~h}^{-1}$. However, he also mentioned that nitric acid is more volatile and hence exists in significant concentrations in the gas phase, while sulphuric acid has a very low vapour pressure under ambient conditions and hence exists in the form of aerosol phase particles.

From the foregoing review, the formation of secondary particles in plumes is shown to be a complex and highly variable process that is influenced by a range of different parameters. However, in general the time scales for the formation of secondary particles via the different conversion mechanisms under various influencing factors such as 
sunlight is long. Since measurements of plume transmissivity based on the current study would be conducted very close to the point of emission, the time required for secondary particles to form should be long relative to the time it takes the combustion products to pass the measurement location. Therefore, sulfates and nitrates are not expected to be present in significant quantities at the measurement location and should not interfere with transmissivity measurements.

\subsection{Summary of background theory}

In summary, soot consists of small spherical primary particles collected into fractal-like, open structured aggregates with a wide range of sizes and shapes. Primary particles that form aggregates are generally small compared to the wavelength of light so that they satisfy the Rayleigh scattering approximation. However, the optical properties of soot aggregates generally are not suited for the Rayleigh or the equivalent volume Mie sphere approximations, and the RDG-PFA method emerges as a good approximation to interpret optical properties in terms of soot concentrations in the plume. In RDG theory, the individual primary particles absorb light independently (i.e., as they would in the Rayleigh limit) and the absorption of light of aggregates is proportional to the volume of the aggregates and insensitive to soot morphology. Therefore, light absorption by aggregates can be used to measure soot concentration. Based on a thermodynamic analysis and literature review, non-soot particles such as water vapour, sulphate and nitrate compound particles should not be present in the target application of plumes of solution gas flares under normal measurement conditions; therefore, they should not interfere with the measurements. 
Through use of the RDG-PFA scattering approximation in conjunction with Beer's law, it is shown that the soot mass emission rate can be functionally related to plume transmissivity. Plume transmissivity is measured using the 2D-LOSA optical diagnostic technique. In the lab implementation of 2D-LOSA, three (3) images are captured to measure the transmissivity, referred to as a 3-image method. To suit a field application of 2D-LOSA where sky radiation is used as the light source, a single-image method is introduced in which the background intensity behind plume can be predicted through interpolation from regions of the image beyond the plume. The single-image method implementation for different sky conditions are presented in Chapter 4. In the following chapter, the experimental apparatus of 2D-LOSA for different light sources and steady soot sources are discussed with a focus on plume transmissivity measurements. 


\section{Experimental Apparatus}

The optical diagnostics used to make quantitative transmissivity measurements are described in this chapter. Three main experimental configurations were utilized to permit analysis and comparison of experiments with different light sources. The three sources were collimated light from an arc lamp, diffuse light from an arc lamp coupled with an integrating sphere, and sky-scattered solar radiation. The sections below detail the experimental apparatus layouts with the three light sources as well as lens settings, burners, and artificial test plumes. Section 3.5 also provides a brief explanation of the Scanning Mobility Particle Sizer used to help establish baseline experimental conditions.

\subsection{Collimated Light 2D-LOSA Setup}

A Line-of-Sight Attenuation (LOSA) technique is used for the determination of soot volume fractions by light extinction measurement. LOSA is an established non-intrusive optical method for soot concentration measurement. In LOSA measurements, the transmissivity of a soot-laden medium (i.e., plume) is measured along a linear path through the medium [Thomson, 2008b]. In traditional or 0-D LOSA, a laser beam is passed through the medium and the intensity of the beam is measured before and after the medium using photodiodes. To fully characterize a $2 \mathrm{D}$ medium, the medium must be moved relative to the laser to obtain a library or map of transmissivities [Gore and Faeth, 1986]. The process can be highly time consuming and increases the susceptibility of the 
diagnostic to measurement noise and variation in the medium during measurement. The two-dimensional line-of-sight attenuation (2D-LOSA) technique is an implementation of LOSA where an extended light source and two-dimensional detector array are used to allow multiple parallel LOSA measurements at a single time, thus allowing a full simultaneous mapping of a medium. Two-dimensional LOSA was first performed with an expanded laser beam as the light source by Greenberg and $\mathrm{Ku}$ [Greenberg and $\mathrm{Ku}$, 1997]. Snelling et al. (1999) used a collimated light source generated by an arc lamp (referred as collimated 2D-LOSA) to avoid the spectral coherence of laser sources.

Collimated 2D-LOSA, based on the optical setup developed in [Snelling et al. 1999], was the starting point of plume transmissivity experiments. Figures 3.1 shows the collimated light setup used in this thesis. The emission of an Hg arc lamp A is focused by lens B onto aperture C. The light passing through this aperture is then collimated with lens $\mathrm{E}$ and passes through the plume. The post plume optics consist of a neutral density filter pack $\mathrm{H}$, a band pass filter $\mathrm{I}$, and a charge-coupled device (CCD) camera $\mathrm{K}$, with a commercial camera lens J. The neutral density filters are selected so that the unattenuated lamp light fills the dynamic range of the detector. The detector is an aircooled 1100 x 330 pixels CCD Princeton TEA/CCD-1100 PF M 059616 with a Model ST 138 controller. The CCD pixel size is $24 \mu \mathrm{m} \times 24 \mu \mathrm{m}$. The detector was controlled using Roper Scientific Winview/32 software. The commercial camera lens is focused so that the detector plane and the centre of the plume are optically conjugated in order to minimize the beam steering effect. A picture of the collimated light setup is shown in Figure 3.2. 


\section{Distances:}

$A$ to $B-508 \mathrm{~mm}$

$B$ to $C-508 \mathrm{~mm}$

$C$ to $D-250 \mathrm{~mm}$

$D$ to $E-258 \mathrm{~mm}$

$E$ to $F-320 \mathrm{~mm}$

$F$ to $G-330 \mathrm{~mm}$

$\mathrm{G}$ to $1-300 \mathrm{~mm}$

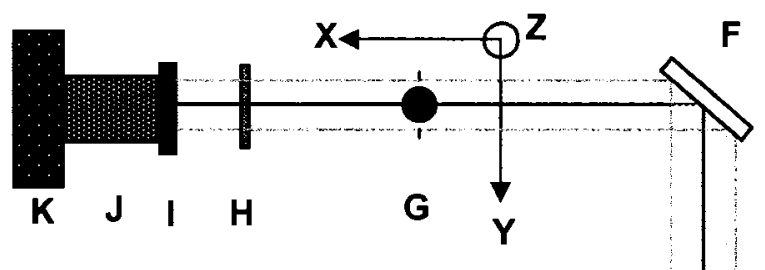

A

B

C

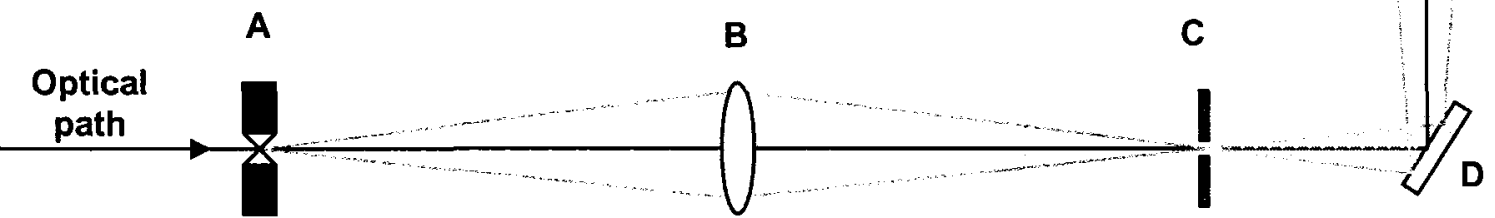

Legend:
A: Oriel model $6821100-W a t t ~ H g$ arc lamp
B: Achromatic lens, $50 \mathrm{~mm}$ D $254 \mathrm{~mm} \mathrm{FL}$
C: Iris diaphragm set to 2.5 to $3 \mathrm{~mm} \mathrm{D}$
D: Front surface mirror $50 \mathrm{~mm} \mathrm{D}$
E: Achromatic lens, $50 \mathrm{~mm} \mathrm{D} 508 \mathrm{~mm}$ FL

G: Test plume

$\mathrm{H}$ : Neutral density filter

I: Narrow band filter, $577 \mathrm{~nm}$

$\mathrm{J}$ : Nikon Camera lens, 105 mm FL

$\mathrm{K}$ : Princeton CCD camera

Figure 3.1: Schematic of optical layout for Collimated LOSA setup

A 20-nm bandwidth filter centred at $577 \mathrm{~nm}$ was used in these experiments to achieve a monochromatic transmissivity measurement. This wavelength was chosen to optimize the lamp emission and avoid interference from other species. Polycyclic Aromatic Hydrocarbons (PAHs) were the primary concern. In the soot formation process (as mentioned in Section 2.1), PAHs are formed in most fuel-rich pyrolysis processes in the lower portion of a flame. In the oxidation region (upper part) of the flame, conversion of fuel to soot by pyrolysis is essentially complete [VanderWal et al., 1997]. Therefore, in the plume region where we are interested, the PAH concentration should be insignificant relative to that of soot. Furthermore, any emission from PAHs predominantly occurs between $300-400 \mathrm{~nm}$ [VanderWal et al., 1997]. Measurements of gas-phase absorption spectra show that $\mathrm{NO}_{2}$ peak absorption occurs around $300-500 \mathrm{~nm}$, 
$\mathrm{SO}_{2}$ is between $240-400 \mathrm{~nm}$, and $\mathrm{CO}_{2}$ and $\mathrm{H}_{2} \mathrm{O}$ are in the infrared regime [Bogumil et al., 2003]. Thus, $577 \mathrm{~nm}$ is a good choice of wavelength for transmissivity measurements designed to detect soot particles only.

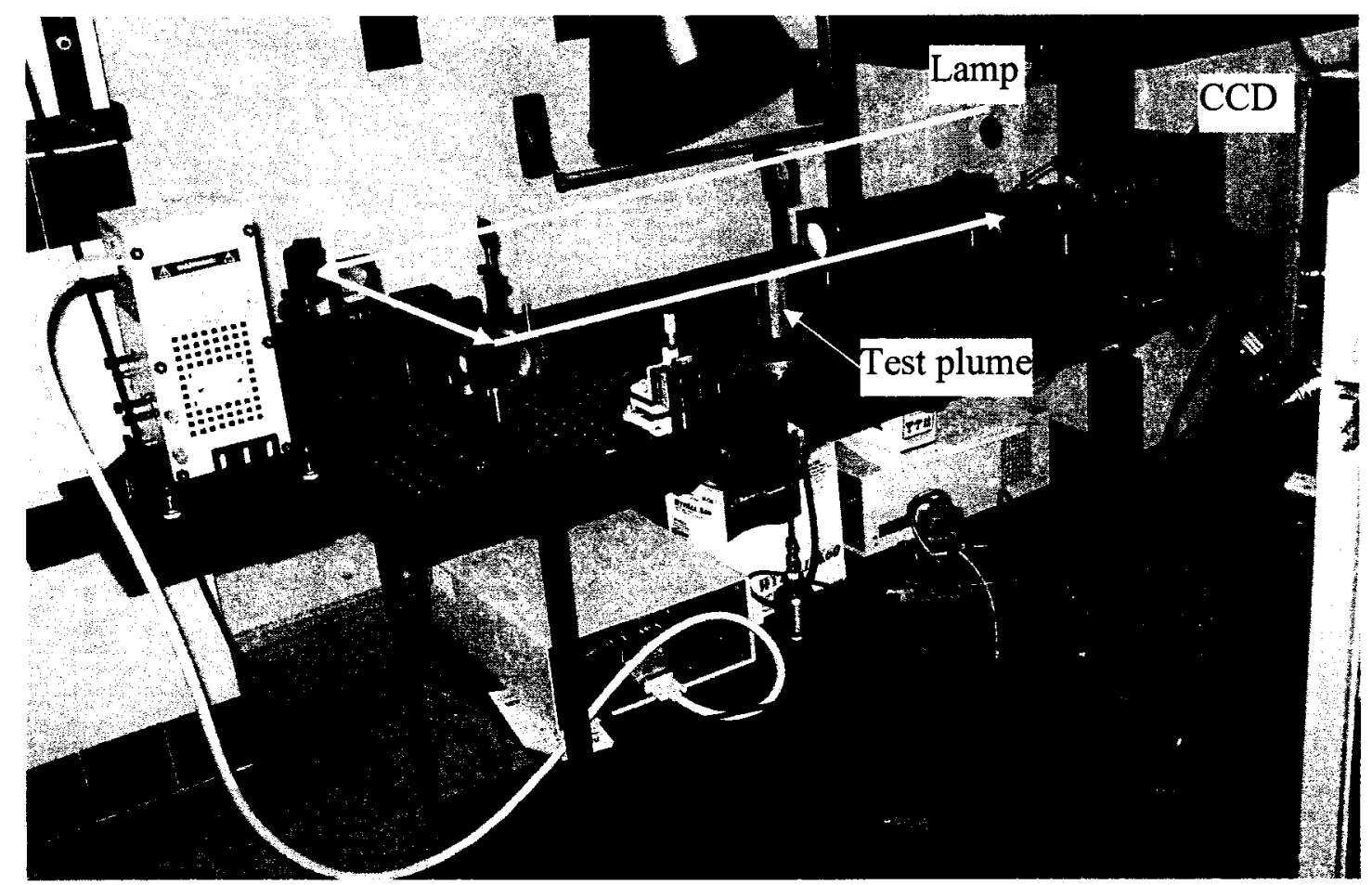

Figure 3.2: Photograph of the Collimated LOSA setup. The yellow lines represent the collimated light path. A second arc lamp relates to a different setup and is not used for the collimated LOSA measurements.

\subsection{Diffuse Light 2D-LOSA Setup}

An alternative implementation of the 2D-LOSA diagnostic that allows improved measurement sensitivity is diffuse 2D-LOSA [Thomson et al., 2008a]. In this diagnostic, an extended diffuse light source is produced by an arc lamp coupled with an integration sphere. The output of the lamp is imaged at the centre of the attenuating medium, which is imaged again by the detection optics. This differs from collimated LOSA where a 
point source is collimated, passed through the attenuating medium and then imaged onto the detector. The technique offers advantages over collimated LOSA because it is less sensitive to defects with the optics and interferences due to beam steering, thus achieving very high levels of sensitivity in transmissivity measurements [Thomson et al., 2008a].

Since skylight is in essence a diffuse extended light source, the measurements using diffuse 2D-LOSA technique provide an ideal reference for verification of measurements obtained using sky 2D-LOSA (described in the next section). The optical setup for diffuse light 2D-LOSA is shown in Figures 3.3 and 3.4.

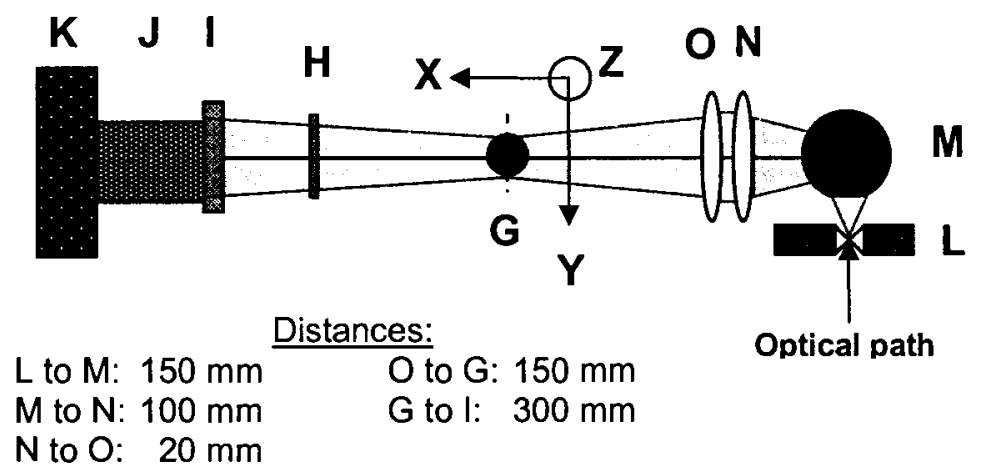

Figure 3.3: Schematic of optical layout for Diffuse LOSA setup 


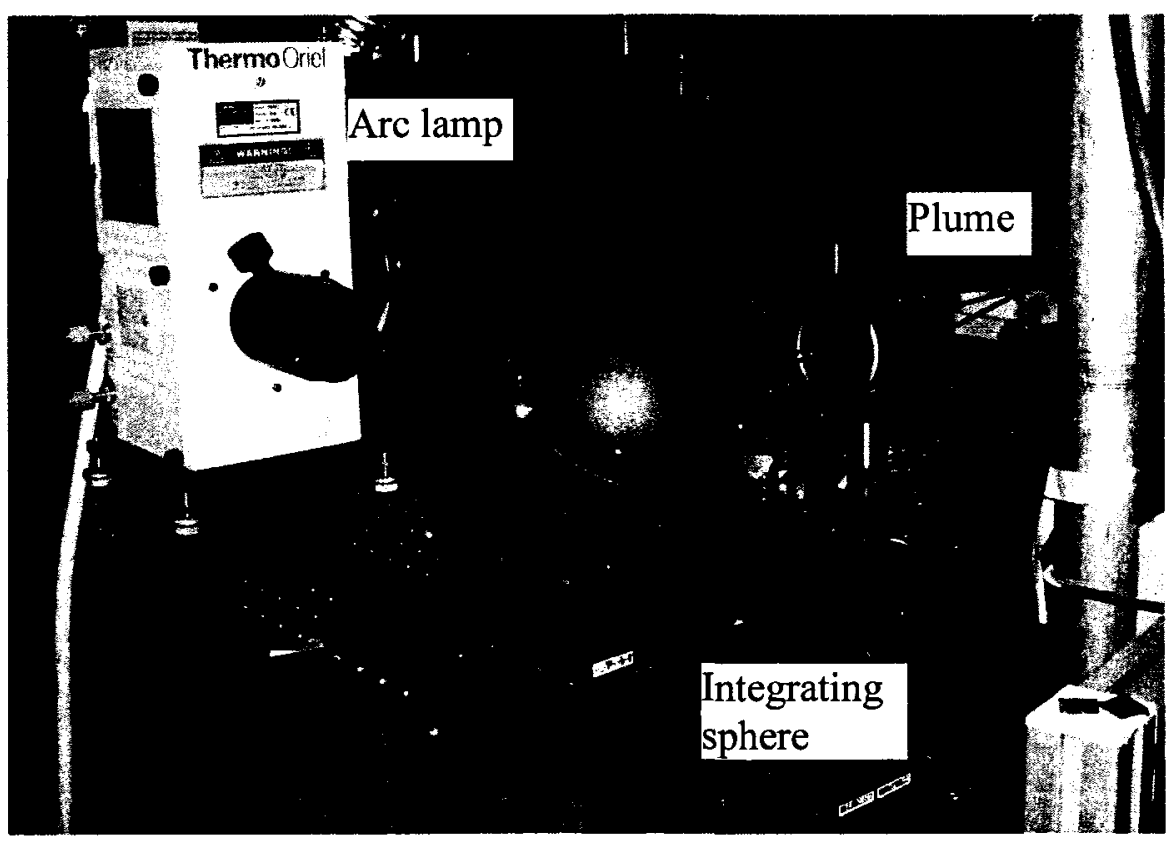

Figure 3.4: Photograph of Diffuse LOSA setup

The arc lamp L and integrating sphere $\mathrm{M}$ produce a 1-inch diameter diffuse light source at the sphere exit port. The plane of the exit port is imaged with a $1.5: 1$ magnification to the centre of the plume using lens $\mathrm{N}$ and $\mathrm{O}$. The plume is then imaged onto the CCD K. The post plume apparatus is the same as that in the collimated 2DLOSA setup; however, the neutral density filter $(\mathrm{H})$ is changed to optimize the light intensity without saturating the camera. Both sets of optical apparatus were installed on a mobile cart, which could be used for outdoor experiments with sky radiation as the light source (refer to Figure 3.2). In the first version of the setup, distances $M N$ and OG (refer to Figure 3.3) were $390 \mathrm{~mm}$ and $390 \mathrm{~mm}$. After initial testing and optimization of the apparatus, these distances were shortened to $100 \mathrm{~mm}$ and 150 as shown schematically in Figure 3.5. The closer arrangement of these lenses effectively shortens the distance between the integrating sphere and the plume so as to increase the collection angle of incident light, $\theta_{1}$, shown in Figure 3.5. 


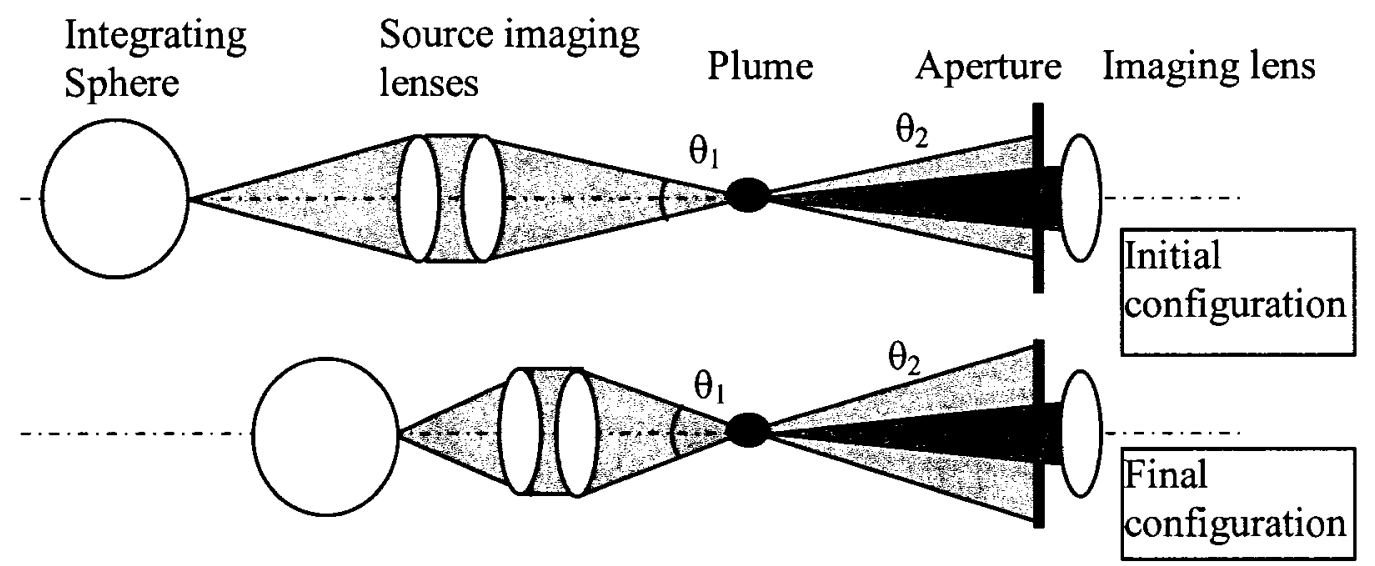

Figure 3.5: Initial and final configurations of source imaging lenses to improve collection ability of the whole system. In the final configuration, the angle $\theta_{1}$ of the light cone passing through the plume (in yellow) is much larger than the angle $\theta_{2}$ of the light cone collected by the imaging lens (in green).

In Figure 3.5, it is shown that after passing through the plume only a smaller portion of the light rays (cone angle of $\theta_{2}$ as shown as green) are imaged onto the imaging lens. $\theta_{2}$ is controlled by the $\mathrm{F} / \#$ of the lens (larger $\mathrm{F} / \#=$ smaller $\theta_{2}$ ) which is described further in Section 3.2.1. If $\theta_{1}$ is much larger than $\theta_{2}$, the light cone region in yellow always covers the region in green, which means that the lens aperture is always filled, even when the light cone (in yellow) shifts due to beam steering. Therefore, the collection ability of the whole system is improved and image distortion is minimized. A more detailed explanation on how a diffuse light system can effectively mitigate interferences due to beam steering can be found in a recent publication [Thomson et al., 2008a], and a simulation of interferences due to beam steering on the current system is presented in Appendix B.

As stated previously, the diffuse light source originates from an integrating sphere. The integrating sphere is an optical component consisting of a hollow cavity with its interior coated with highly reflective material. The light entering the sphere from one 
port bounces around the highly reflective inner surface and finally escapes as a uniform diffuse light source at the exit port as shown in Figure 3.6. The integrating sphere used in the setup was 4-inch diameter with two 1-inch ports.

(a)

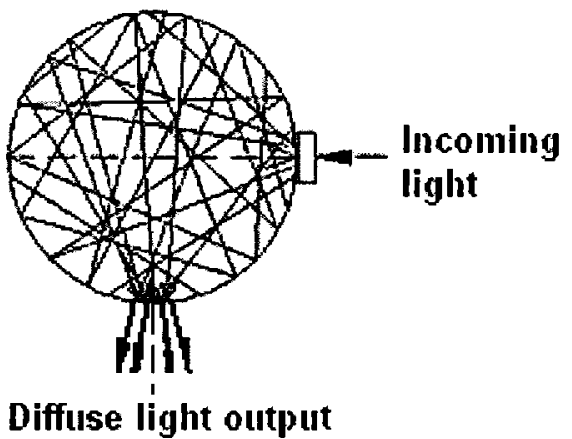

Diffuse light output

Incoming

\section{Figure 3.6: Schematic (a)
3.2.1 Lens and CCD Settings}

\subsubsection{1 $\mathrm{F} / \#$ and aperture size}

The F/\# is a measure of the light collecting capability of the optics and is defined as $\mathrm{F} / \#=f / D$, where $f$ is the focal length and $\mathrm{D}$ is effective diameter [Hecht, 2006]. $\mathrm{F} / \#$ is typically indicated on commercial camera lenses as $\mathrm{F} / 1.4, \mathrm{~F} / 2, \mathrm{~F} / 2.8, \mathrm{~F} / 4, \mathrm{~F} / 5.6, \mathrm{~F} / 8$, $\mathrm{F} / 11, \mathrm{~F} / 16, \mathrm{~F} / 22$, and so on, where each number increases by a multiplicative factor of square root of two $(\sqrt{2})$. Thus, each time the $F / \#$ is increased one 'stop', for example from $F / 5.6$ to $F / 8$, the amount of light collected by the lens halves. A larger F/\# also improves the depth-of-field of the imaging system (the distance in front of and behind the object plane for which objects will appear in focus at the image plane). However, the smaller aperture associated with a larger F/\# significantly reduces the amount of light 
which passes through the lens. Therefore, the F/\# must be set to balance these two effects.

Since the plume image is taken at the visible wavelength of $577 \mathrm{~nm}$, a commercial photographic lens is well suited for this application. A typical commercial camera lens is a compound lens made up of a number of optical lens elements that are designed to correct optical aberrations existing in each lens. In the current setup, a Nikon MicroNikkor $105 \mathrm{~mm}$ focal length camera lens was directly coupled to the CCD and designed to reject spurious light from reaching the imaging plane (as mentioned in Section 2.3.2.2). The $F / \#$ range of the lens was $F / 2.8$ to $F / 22$. After considering both effects mentioned above, an aperture size of $F / 8$ was chosen for the experiments.

\subsection{Sky-LOSA Setup}

Figures 3.7 and 3.8 show the setup for experiments using sky-scattered radiation as a light source. Skylight is collected and reflected by a $1 \mathrm{~m} \mathrm{x} 0.76 \mathrm{~m}$ mirror (labeled $\mathrm{P}$ in Figure 3.7) and is transmitted through the plume. The post plume apparatus is the same as those in both the collimated- and diffuse-light setups, except that the neutral density filter is adjusted to maximize the light intensity without saturating the camera. The detector was positioned relative to the centre plan of the burner exhaust nozzle so that the plume width was less than $25 \%$ of the image width after allowing for some plume movement due to wind effects. 
Legends: $\quad$ Distance:

$P: 1 \mathrm{~m} \times 0.76 \mathrm{~m}$
mirror $\quad$ G to $\mathrm{I}-430 \mathrm{~mm}$

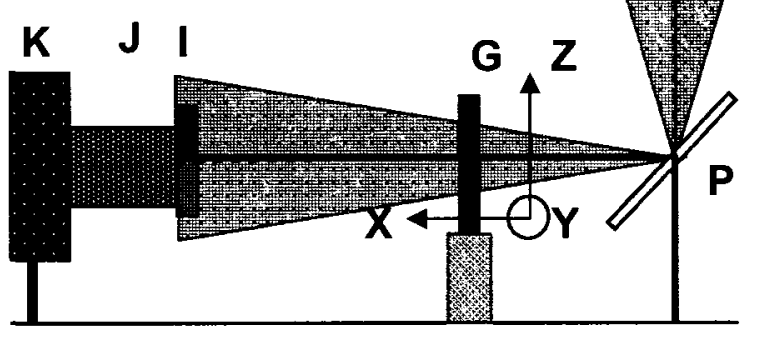

Skylight

Figure 3.7: Schematic of optical layout for sky-scattered LOSA setup.

As can be seen from Figures $3.8 \mathrm{a}$ and $\mathrm{b}$, experiments were conducted in a large garage area so that the majority of the experimental hardware could be sheltered in the garage and in particular, the environmental conditions of the burner could be better controlled to match those of the in-lab diffuse LOSA measurements. During the measurements, the large garage door was fully opened, and a large $(1 \mathrm{~m}$ by $0.76 \mathrm{~m})$ flat back-surface, commercial grade mirror was positioned just outside the lab and aligned with the burner tip and the camera to reflect the skylight through the plume into the optical system. Additionally, a black screen was positioned to prevent the sun from directly shining onto the system.

Since measurements were performed in winter, the lab temperature did drop significantly when the garage door was opened, which could have led to differences of plume temperatures and different soot concentrations between diffuse and sky-LOSA measurements for the same test conditions. 

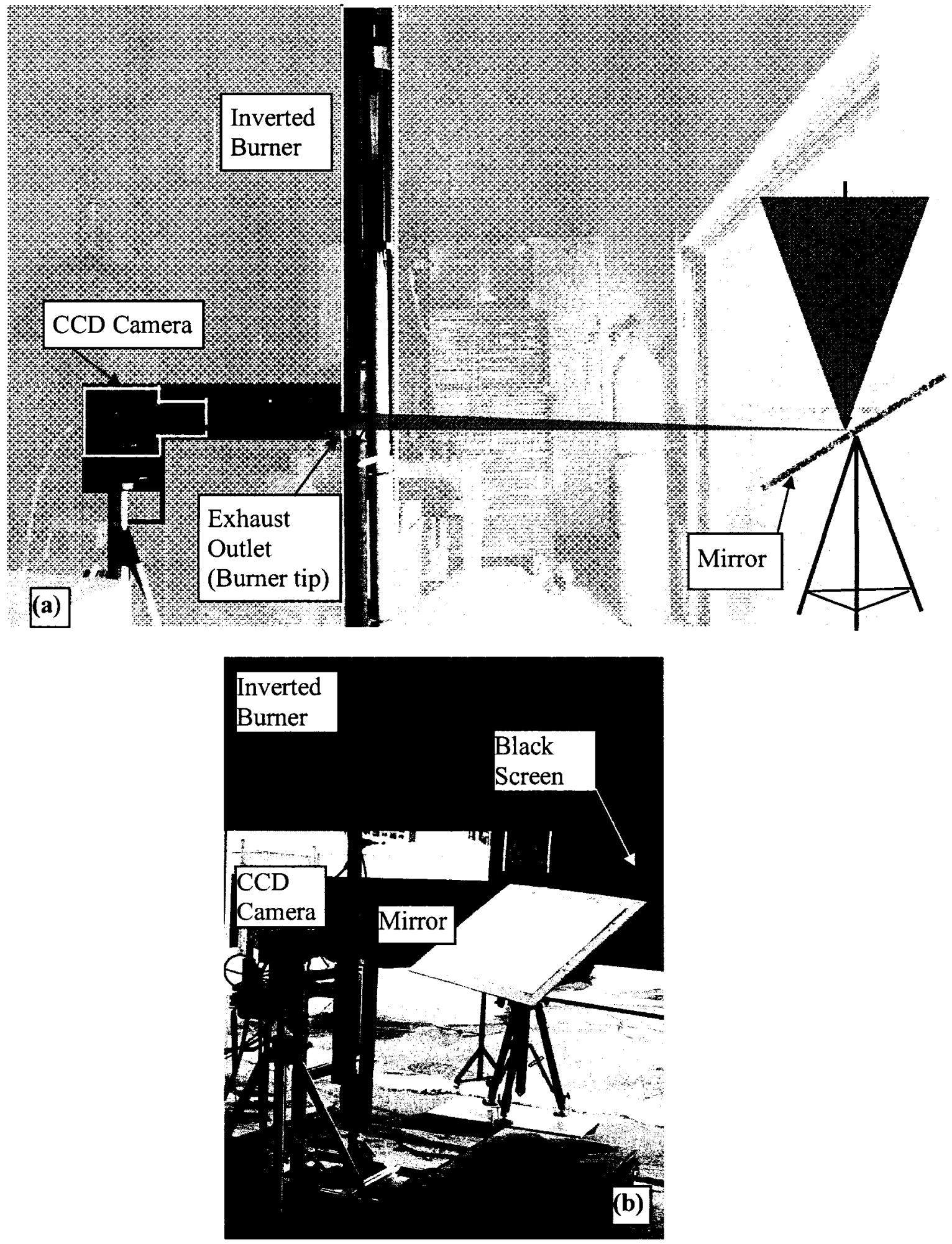

Figure 3.8: a) Sky-scattered light LOSA experimental setup b) another view of the same setup. 


\subsection{Soot Sources for Transmissivity Experiments}

Three soot sources were used in different stages of the work: a co-flow laminar nonpremixed burner, artificial plumes consisting of soot deposited onto microscope slides, and an inverted co-flow burner. The co-flow laminar non-premixed burner was initially utilized as a soot source. Due to a time-varying behaviour of the burner, this burner was replaced by artificial test plumes (i.e., soot from the co-flow laminar non-premixed burner thermophoretically deposited onto glass microscope slides). These synthetic test plumes were invariant in time, but reflections from four sides of the glass slides was also a source of noise that interfered with measurements. An inverted co-flow diffusion burner capable of producing steady plumes of cooled soot was used as the third and final soot source for the attenuation measurement.

\subsubsection{Co-flow Laminar Non-premixed Burner}

A co-flow laminar non-premixed burner was used to generate a soot source during preliminary tests as shown in Figure 3.9. The fuel was acetylene and issued at $37 \mathrm{sccm}$ (sccm denotes cubic centimeters per minute at standard temperature and pressure) from a central fuel tube with a diameter of $3.06 \mathrm{~mm}$. The co-flow air was supplied at $13.2 \mathrm{slpm}$ (slpm denotes liters per minute at standard temperature and pressure) from a co-annular tube with a diameter of $25.4 \mathrm{~mm}$. The air flow was straightened using a combination of glass beads and sintered metal foam. A chimney with glass windows for optical access was used to protect the plume from air movement in the room. 

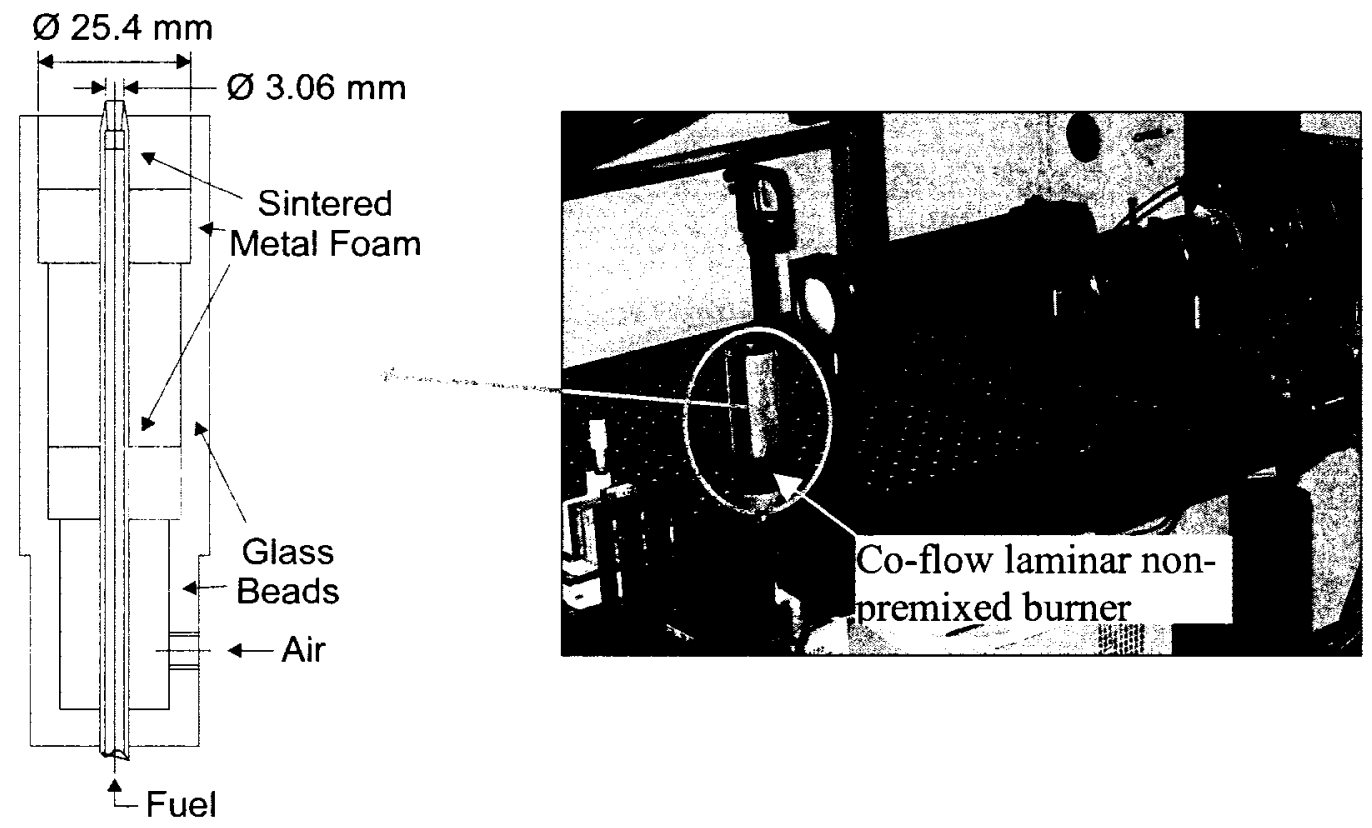

\section{Figure 3.9: Co-flow laminar non-premixed burner. The schematic figure was adapted from [Thomson et al., 2005]}

Due to some measurement irregularities observed during preliminary characterization of the various light sources, it was suspected that the output of the burner was not stable with time. Tests were performed to look at the stability of the burner and results of this test are shown in Fig 3.10. Since the quantity of $\int \ln \left(\tau_{\lambda}(y)\right) d y$ (integral of transmissivity across the plume width) is proportional to the soot emission rate $\dot{m}_{\text {soot }}$ (as shown in Equation 2.16), this quantity is used here for the stability test. It is clear from the tests that this burner cannot generate a steady soot source within a one hour time period. This time-varying behaviour was believed to be due to soot accumulation at the tip of the burner that could not be avoided, and therefore its use was discontinued. 


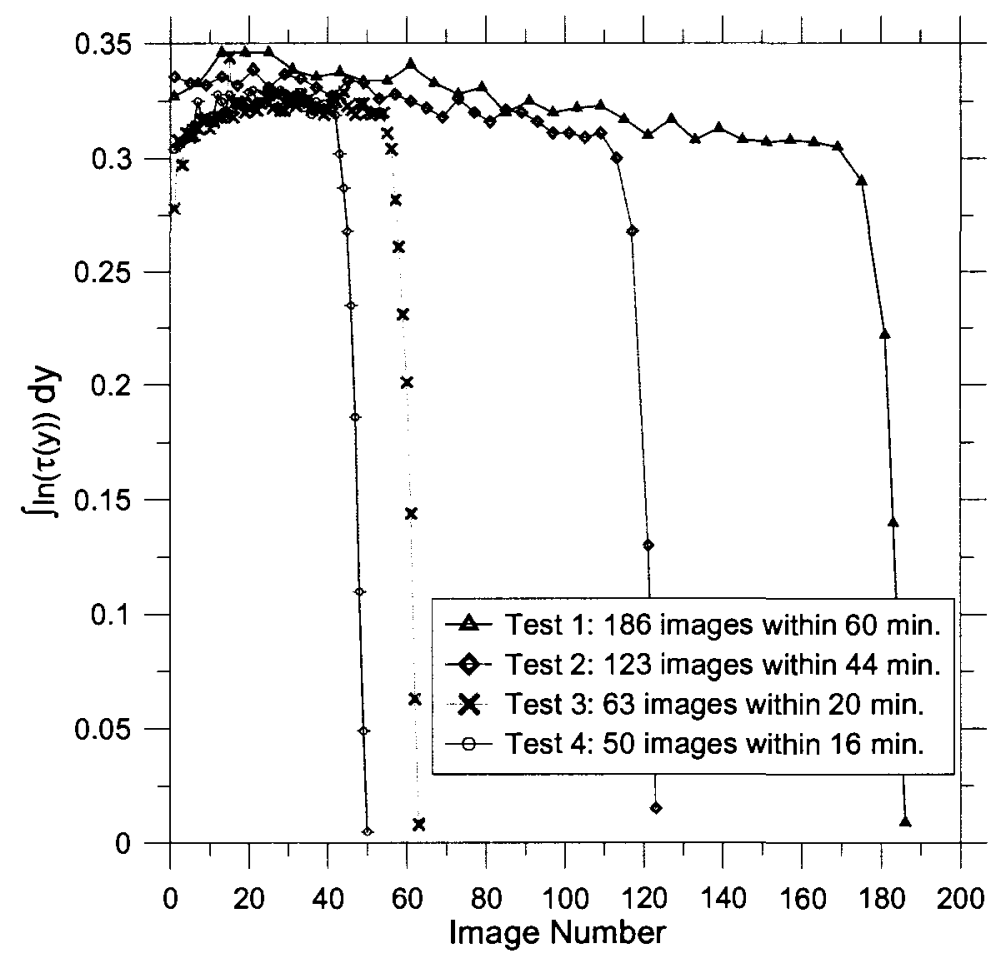

Figure 3.10: Soot source stability test for co-flow laminar non-premixed burner.

\subsubsection{Artificial Plumes}

Due to the problems with time-varying behaviour of the first soot source, a need to create a highly repeatable measurement source was identified. Rather than trying to produce a plume of soot from a flame, soot was instead collected onto a glass slide which was then used as a surrogate for an actual soot plume. To create the artificial plumes, a piece of microscope slide was passed through a soot plume produced from the acetylene flame discussed above. Due to the temperature gradient between the plume and the glass, small particles of soot migrated from the plume and accumulated on the slide. To protect the deposited soot on the slide, a second microscope slide was affixed to the first using double-sided tape, thus creating an air gap between the two slides with the soot deposited on one of the inner glass surfaces. By adjusting the flow rate of burner and the 
immersion time of the slide in the plume, seven sample plumes were made with the transmissivity, $\tau_{\lambda}$, ranging from 0.4 to 0.983 as shown in Table 3.1.

Table 3.1 Associated transmissivities of test plumes.

\begin{tabular}{|c|c|c|}
\hline Test plumes & Transmissivities & Average transmissivities \\
\hline Plume 1 & 0.4 & 0.4 \\
\hline Plume 2 & $0.54-0.64$ & 0.59 \\
\hline Plume 3 & $0.87-0.91$ & 0.89 \\
\hline Plume 4 & $0.91-0.949$ & 0.929 \\
\hline Plume 5 & $095-0.96$ & 0.955 \\
\hline Plume 6 & $0.97-0.98$ & 0.975 \\
\hline Plume 7 & $0.98-0.985$ & 0.983 \\
\hline
\end{tabular}
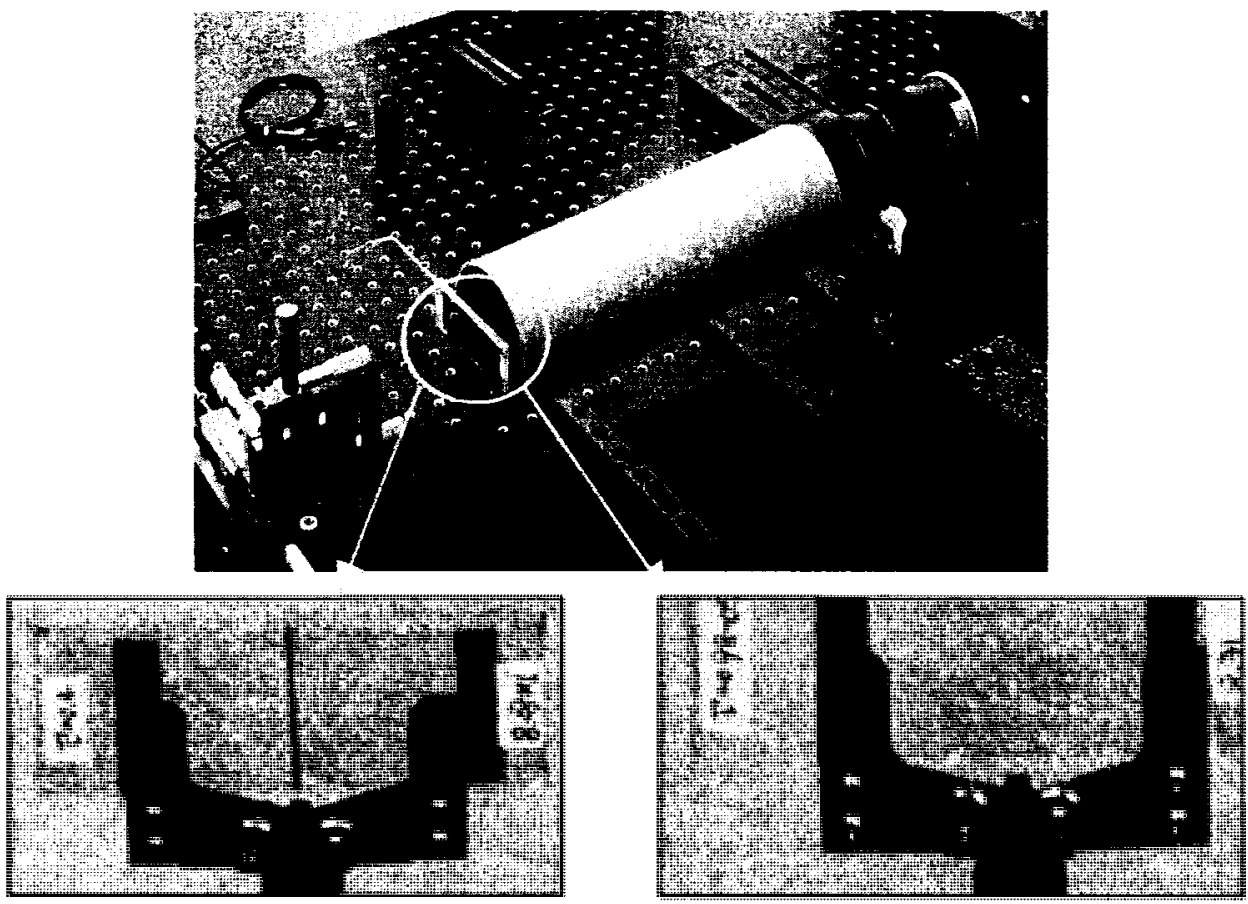

Figure 3.11: Artificial test plumes made by thermophoretically depositing soot onto microscope slides. Test plumes transmissivities a) $\tau_{\min }=0.4$ b) $\tau_{\max }=0.983$.

The synthetic test plume provides an entirely repeatable attenuating medium to test the various light sources; however, measurements of the synthetic plumes made using the different light sources compared poorly for relatively modest transmissivities. It is suspected that reflections from the four sides of the two slides interfered with the 
measurements and adversely influenced the minimum detectable limit in plume transmissivity measurements.

\subsubsection{Inverted Co-flow Non-premixed Burner}

An inverted co-flow non-premixed burner was used as the third and final soot source for the attenuation measurement. The burner was developed by Coderre et al. (2007) following a similar design developed by Stipe et al. (2005) and more detailed descriptions of the burner can be found within those references. The burner is shown schematically in Figure 3.12a and as a photograph in Figure 3.12b.

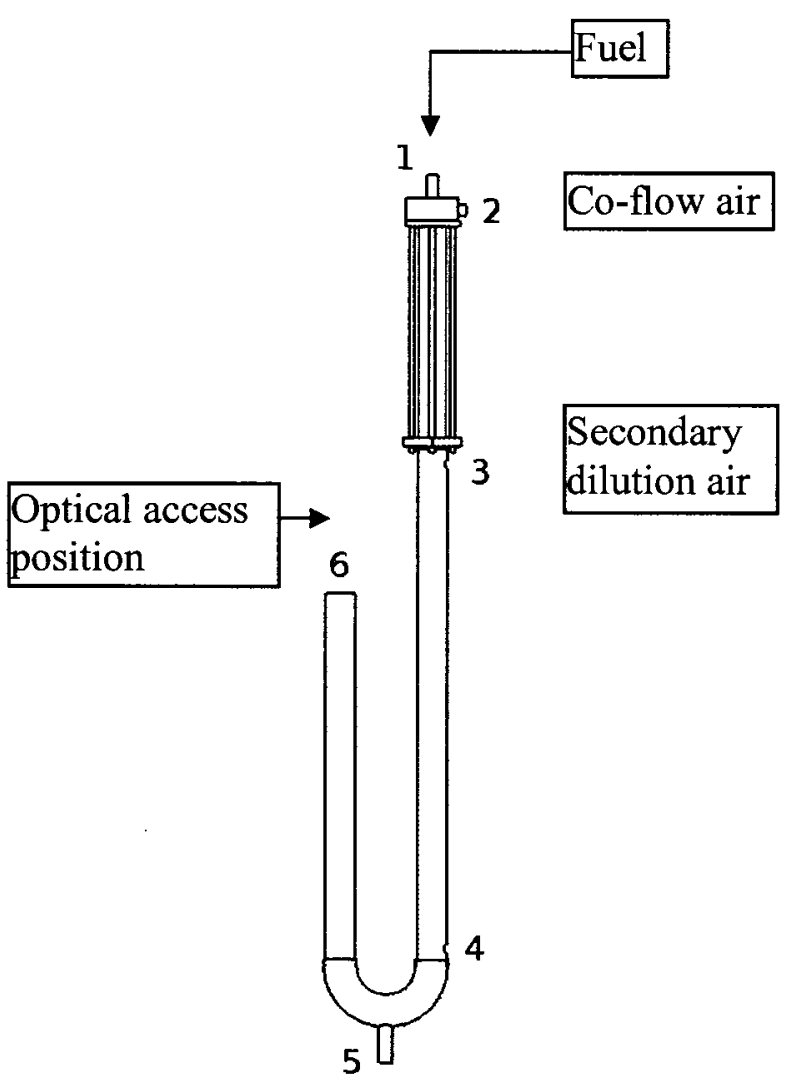

(a)

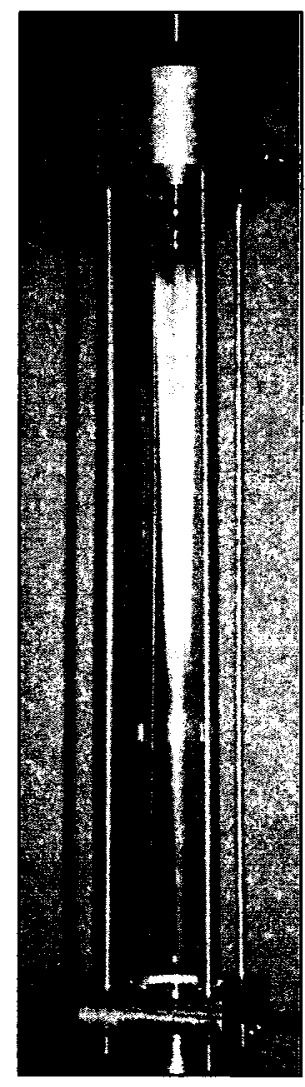

(b)

Figure 3.12: a) Schematic of the inverted co-flow burner b) Photograph of burner head 
Fuel was brought into the burner through port 1 (see Figure 3.12 a) and the oxidizer, co-flow air, was introduced into the annular region between the fuel jet and the outer quartz tube from port 2. Variable amounts of secondary dilution air were injected through port 3 to dilute, cool, and mix the exhaust. With the inverted design, the upward buoyant force from hot combustion products maintains a uniform downward flow in the burner, thus stabilizing the flame [Stipe et al., 2005]. Figure 3.13 shows the number concentration for five (5) consecutive runs measured using a Scanning Mobility Particle Sizer (SMPS), taken at one burner condition, which illustrates the stability of the burner system with time.

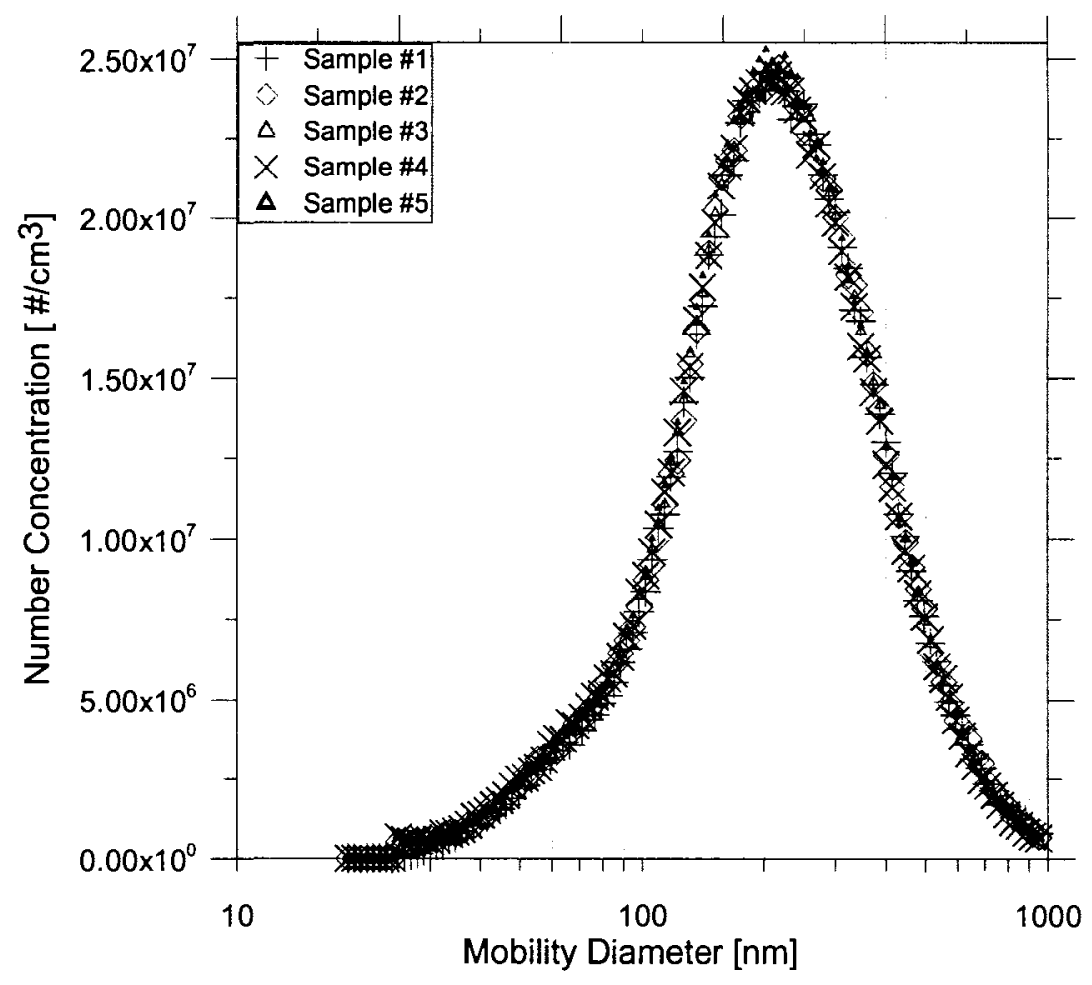

Figure 3.13: SMPS scans results at a burner condition of fuel $\left(\mathrm{CH}_{4}\right)$ flow rate of 1.2 slpm, co-flow air flow rate of $15 \mathrm{slpm}$, and dilution air flow rate of $50 \mathrm{slpm}$ in $\mathrm{log}$ scale [Coderre et al., 2007]. 
The optical diagnostic is applied to the plume at the region above the exhaust outlet (port 6) where it is well mixed. However, since the diagnostic makes integrated measurements across the plume cross-section, it is not necessary that the plume is uniform. The soot concentration in the plume is controlled by adjusting the relative amounts of fuel, co-flow air, and secondary dilution air. Different configurations of the flow rates of fuel, co-flow air, and dilution air were tested. The corresponding plume transmissivities were determined using in-lab diffuse LOSA, as shown in Figure 3.14. From the figure, the overall equivalence ratio calculated for Test 1 (the lowest air flow rate of $14.5 \mathrm{slpm}$ ) was 0.8 , i.e., all flames were over-ventilated. The six flow configurations bracketed within orange circles were selected to provide a range of plume transmissivities to evaluate the sky-scattered LOSA diagnostic. 


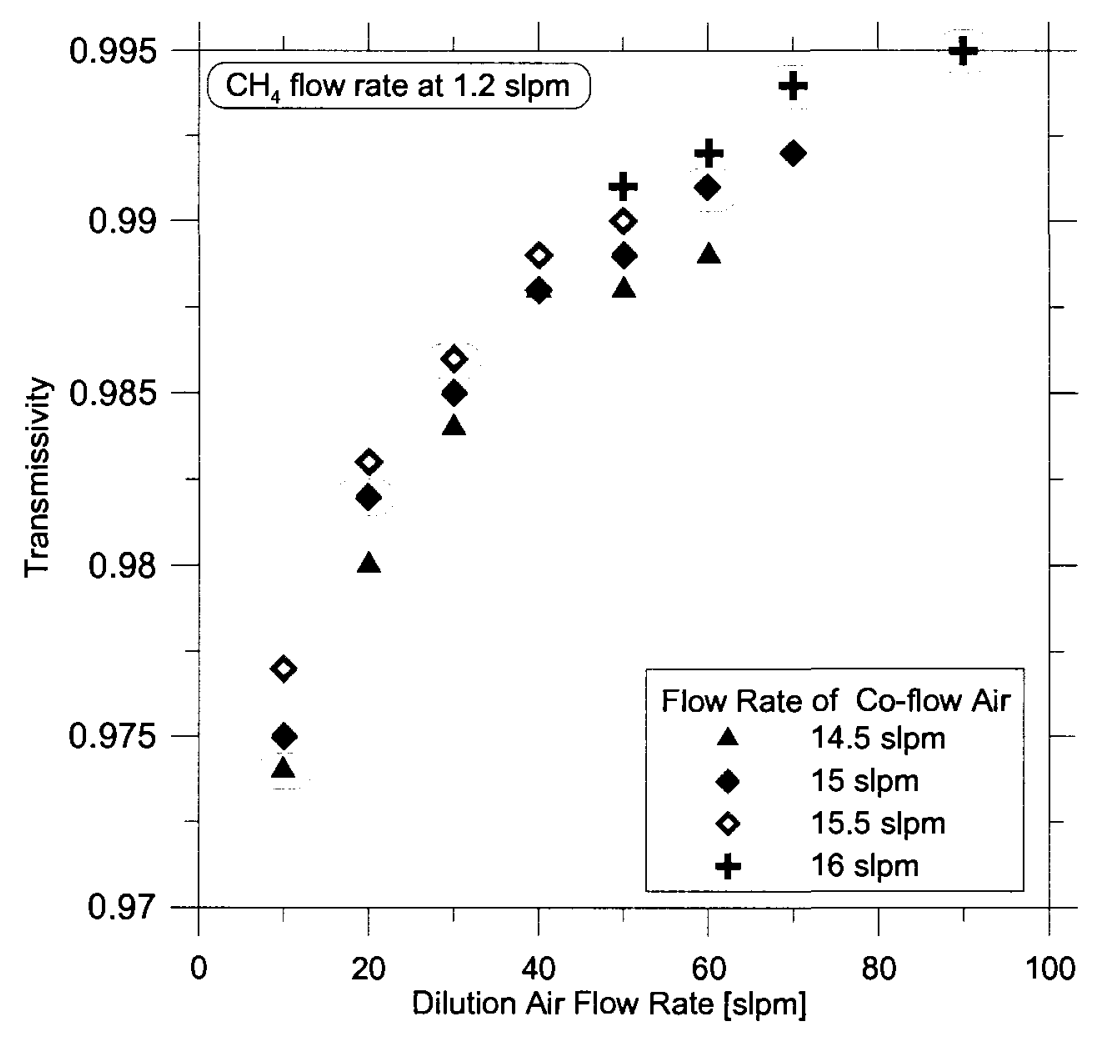

Figure 3.14: Transmissivities of plumes generated under different flow rates of fuel, co-flow air, and dilution air. The transmissivities were determined using the in-lab diffuse 2D-LOSA diagnostic.

Table 3.2 lists the different tests that were evaluated in the final experiments. The transmissivity values corresponding to each test were obtained from in-lab diffuse LOSA measurement. Table 3.3 lists the different weather conditions under which the tests were repeated. 
Table 3.2: Burner operating conditions corresponding to the plume transmissivities

\begin{tabular}{|c|c|c|c|c|c|}
\hline $\begin{array}{c}\text { Test } \\
\text { Conditions }\end{array}$ & $\begin{array}{c}\mathrm{CH}_{4} \text { flow } \\
\text { rate } \\
\text { [s/pm] }\end{array}$ & $\begin{array}{c}\text { Co-flow air } \\
\text { flow rate } \\
\text { [s/pm] }\end{array}$ & $\begin{array}{c}\text { Dilution air } \\
\text { flow rate } \\
\text { [slpm] }\end{array}$ & $\begin{array}{c}\text { Plume } \\
\text { transmissivity }\end{array}$ & $\begin{array}{c}\text { Maximum } \\
\text { Particle } \\
\text { Mobility } \\
\text { Diameter } \\
\text { [nm] }\end{array}$ \\
\hline Test 1 & 1.2 & 14.5 & 10 & 0.974 & 224 \\
\hline Test 2 & 1.2 & 15 & 20 & 0.982 & 217 \\
\hline Test 3 & 1.2 & 15.5 & 30 & 0.986 & 202 \\
\hline Test 4 & 1.2 & 15.5 & 60 & 0.991 & 202 \\
\hline Test 5 & 1.2 & 16 & 70 & 0.994 & 209 \\
\hline Test 6 & 1.2 & 16 & 90 & 0.995 & 209 \\
\hline
\end{tabular}

Table 3.3: Different weather conditions

\begin{tabular}{|c|c|c|c|}
\hline & Clear sky & Cloudy sky & Overcast sky \\
\hline Temperature & $-14^{\circ} \mathrm{C}$ & $-12^{\circ} \mathrm{C}$ & $-10^{\circ} \mathrm{C}$ \\
\hline Pressure & $102.19 \mathrm{kPa}$ & $102.98 \mathrm{kPa}$ & $102.74 \mathrm{kPa}$ \\
\hline Wind Speed & $19 \mathrm{~km} / \mathrm{h}$ & $15 \mathrm{~km} / \mathrm{h}$ & $20 \mathrm{~km} / \mathrm{h}$ \\
\hline Relative Humidity & $46 \%$ & $56 \%$ & $52 \%$ \\
\hline Visibility & $24 \mathrm{~km}$ & $24 \mathrm{~km}$ & $24 \mathrm{~km}$ \\
\hline
\end{tabular}

\subsubsection{Characterization of Soot Aggregates Emitted by the Inverted Burner}

As mentioned in Chapter 2, soot is depicted as fractal aggregates made up of primary particles. To convert optical transmissivity measurements for a plume to soot concentrations using RDG-PFA theory, the primary particles in aggregates have to fall in the Rayleigh range (i.e., the primary particle size parameter $x_{\mathrm{P}}=\pi d_{\mathrm{P}} / \lambda<0.3$ ) and the extinction measurements must be corrected for scatter. However, both measuring the diameter of a primary particle $\left(d_{\mathrm{p}}\right)$ and calculating the ratio of scattering to absorption coefficient $\left(\rho_{\mathrm{sa}}\right)$ require detailed knowledge of the soot morphology including aggregate size distribution and fractal parameters of the aggregates. These can be obtained through a combination of gravimetric sampling, electron microscopy, and LOSA measurements.

Measuring soot morphology and calculating scatter corrections are not the main concern for this project. Fortunately, Coderre et al. (2008) characterized the soot 
morphology of soot emitted from the burner used in this project at an intermediate test condition (fuel flow rate of $1.2 \mathrm{slpm}$, co-flow air flow rate of $15 \mathrm{slpm}$, and dilution air flow rate of $50 \mathrm{slpm})$. At this test condition,

- The primary particle diameter was $33.2 \mathrm{~nm}$ with a standard deviation of $5 \mathrm{~nm}$.

- Fractal prefactor $\left(k_{\mathrm{f}}\right)$ of the aggregates was 1.60 and the fractal dimension $\left(D_{\mathrm{f}}\right)$ was 1.74 .

- The peak mean mobility diameter of aggregates was approximately $200 \mathrm{~nm}$.

Based on the above soot morphology information, $\rho_{\mathrm{sa}}$ was calculated to be 0.12 at the wavelength of $577 \mathrm{~nm}$ [Coderre et al., 2008].

These values were not expected to vary substantially for the range of test conditions outlined in Table 3.2. To verify this assumption and in lieu of the soot morphology information for each of the test conditions, electrical mobility diameters of the aggregates were measured using a Scanning Mobility Particle Sizer (SMPS). SMPS is a diagnostic that provides an indication of particle size distribution. Measured electrical mobility diameters at each condition were compared with that measured from the wellcharacterized test condition.

\subsubsection{Scanning Mobility Particle Sizer (SMPS) Measurements of Soot from the Inverted Burner}

The SMPS measures the size distribution of particles using an electrical mobility detection technique. The electrical mobility is a measure of the particle's ability to move in an electric field. The SMPS consists of two main systems: a Differential Mobility Analyzer (DMA) which separates particles based on their electrical mobility and a Condensation Particle Counter (CPC) to measure the particle number concentration. 
After particles passing through a bipolar charger, a positive charge is established on the particles. The charged particles then feed into the Differential Mobility Analyzer (DMA). The DMA contains two concentric metal cylinders. The inner cylinder, the centre rod, is maintained at a controlled negative voltage, while the outer cylinder is electrically grounded. This creates an electric field between the two cylinders. The particles and sheath air are introduced at the top and flow down the annular space between the cylinders. The particles surround the sheath air, and both flows pass down the annulus with no mixing of the two laminar streams. The electric field causes positively charged particles to be attracted through the sheath air to the negatively charged centre rod. Particles within a narrow range of electrical mobility exit through a small slit located at the bottom of the centre rod. These particles are transferred to the Condensation Particle Counter (CPC) to determine the particle concentration [Aerosol Instrument Scanning Mobility Particle Sizer Manual, 2006].

For the range of test conditions in Table 3.2, the peak mean mobility diameters varied from $202 \mathrm{~nm}$ to $224 \mathrm{~nm}$ (refer also to Figure 3.15) as compared to $200 \mathrm{~nm}$ at condition measured by Coderre et al. (2008). Based on these results, the values of $\rho_{\text {sa }}$ under current test conditions should be in the same order as that in Coderre's test condition. Therefore, light scattering from aggregates is not significant in the present experiments. Furthermore, current experiments are conducted to directly compare results of outdoor sky-scattered solar radiation measurements with in-lab diffuse-LOSA measurements for the same test conditions. Ideally, the values of $\rho_{\mathrm{sa}}$ should be constant at each test condition for both measurements. 


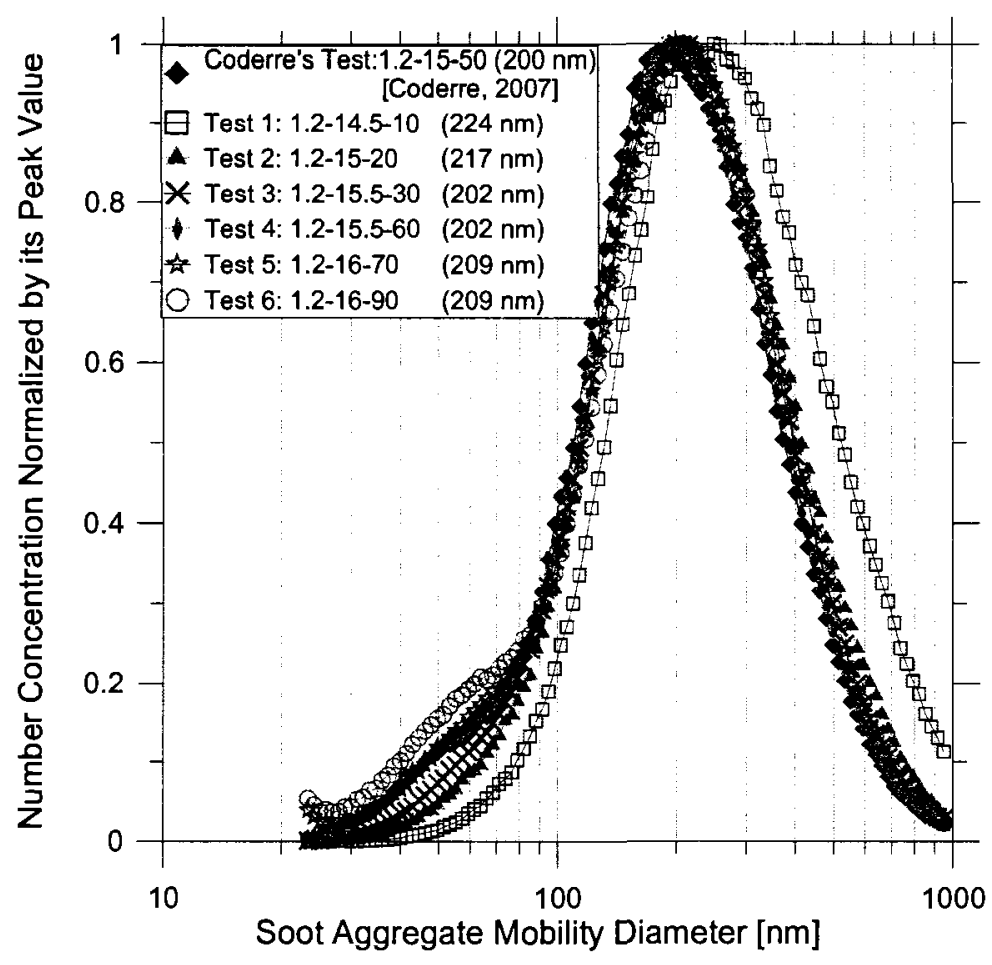

Figure 3.15: Number concentration normalized by its peak value vs. soot aggregate mobility diameter.

\subsection{Summary of Experimental Apparatus}

The experimental apparatus used for the plume attenuation measurement under different light sources has been outlined. The inverted co-flow diffusion burner was determined to be a steady soot source for the final experiment. For lab-based measurements using either collimated- or diffuse 2D-LOSA, transmissivity was measured from a sequence of three monochromatic measurements, where the background image was obtained in the absence of the attenuating medium. However, for 2D sky-LOSA, the background image has to be determined from interpolation. In the following chapter the method for generating an interpolated background is presented and the accuracy of the method is evaluated. 


\section{Results: Sensitivity and Uncertainty from Background Analysis}

\subsection{Interpolation for Background Intensities Behind the Plume}

\subsubsection{Background Interpolation Algorithm}

In a laboratory implementation of 2D-LOSA, it is possible to obtain a measurement of $I_{0}$, the background image, by simply turning off or redirecting the soot source away from the measurement volume. By using a steady light source and an integrating sphere, the light field is highly repeatable between the transmission and background images and thus a good measure of the transmissivity through the plume is possible. However, in a field application of 2D-LOSA, where sky radiation is the light source for the measurement of plume transmissivity, it is impossible to isolate the background light from the attenuating medium since the plume cannot be 'turned off'. Furthermore the sky intensity varies strongly with time and position. Therefore, an interpolated background must be generated from a single "transmission image" to permit calculation of the plume transmissivity, in a process known as the 1-image method.

To generate the interpolated background, the section of an image where the plume is present is removed, and the intensity data on either side of the plume image location is used to interpolate a synthetic background in the region of the plume. In the early stages of the investigation, different interpolation methods were considered, specifically 1D and 2D polynomial regression and 1D Loess smoothing, which relies on a locally weighted 
least square method to fit a quadratic profile to measured intensity data. The Loess algorithm was determined to be most appropriate for this application and interpolation was performed using a MathCAD implementation of the Loess algorithm. Essentially, the Loess function fits a second order polynomial to the neighbourhood of points surrounding the location to be evaluated. The span argument of the Loess function controls the range of the neighbouring data points used in the calculation for a given data point [MathCAD, v.13, PTC, Needham, MA]. The span is specified as the fraction of the total number of data points included in the local fit. For example, a span of 0.1 signifies that $10 \%$ of the actual data points are used to do the fit. A large span increases the smoothness but decreases the spatial resolution of the data interpolation, while a small span decreases the smoothness but increases the spatial resolution of the data interpolation. The optimal span value depends on the data set, and usually requires experimentation to determine.

At the start of the investigation, the accuracy of the background interpolation for different light sources such as collimated, diffuse, and skylight source was investigated. For each source, 30 background images were collected (i.e., images where no attenuating medium is present). The background prediction algorithm was tested by removing a vertical section of each image where a plume would be present and then quantitatively comparing the interpolated background with the actual measured background. In the absence of a plume, the interpolated background should ideally match the actual background. Figure 4.1 shows absolute and relative comparisons of actual and interpolated background intensities for the three light sources. 

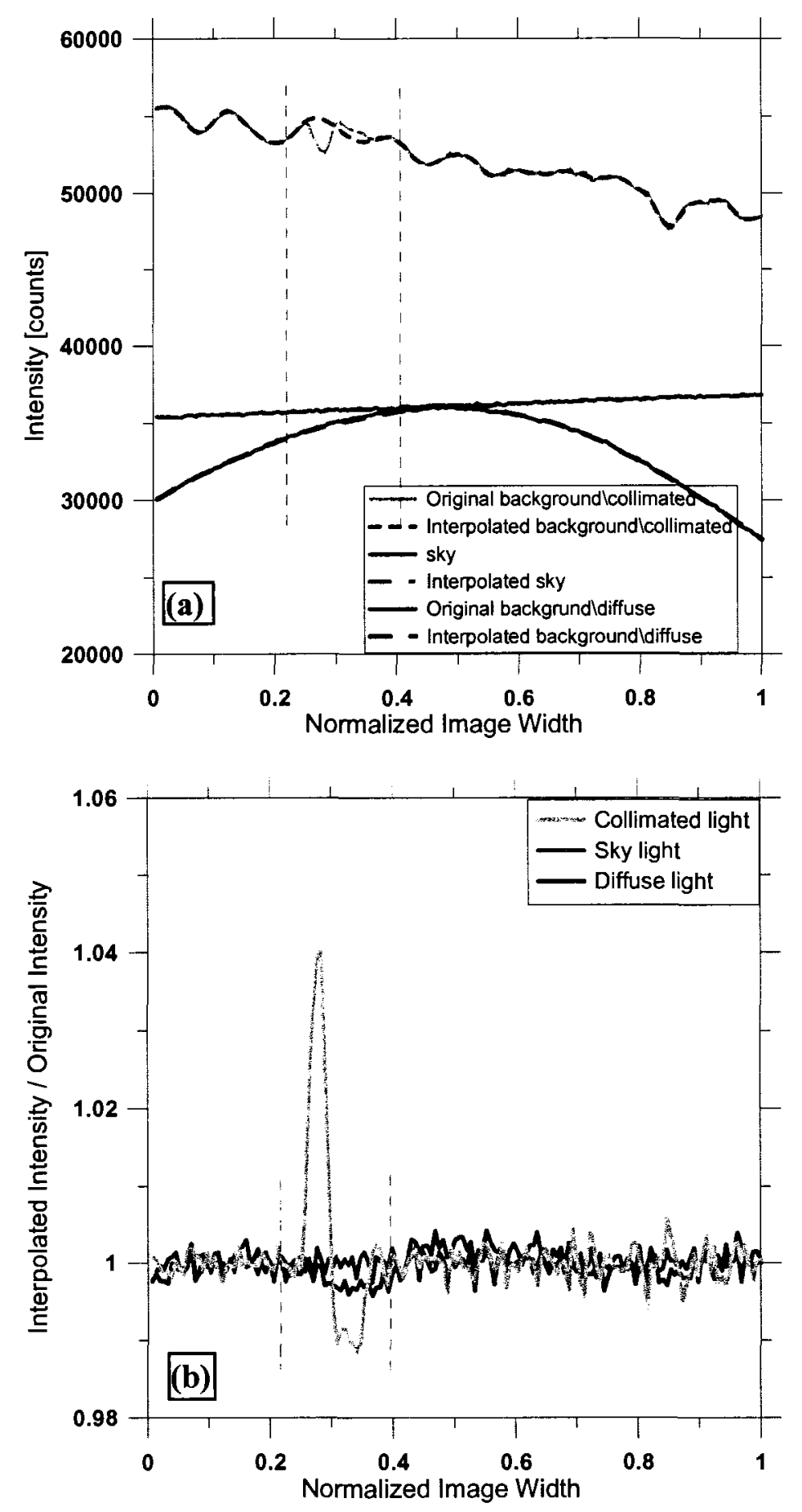

Figure 4.1: Comparison of interpolated and actual background for different light sources. Vertical dashed lines indicate region of interpolation. (a) Plots of absolute intensity and (b) normalized intensity.

As shown in Figure 4.1a, the agreement between the actual and interpolated background intensities is very good within the plume region (the region between dotted 
lines) for diffuse and skylight, although less good for collimated light. The difference between the actual and interpolated background is quantified by taking the ratio of light intensity of the interpolated background to the actual measured intensity for each point over the whole image width and for each different light source. If the interpolated intensity in the plume region is exactly the same as the measured one, the ratio of these two intensities should be unity. It is observed from Figure $4.1 \mathrm{~b}$ that the ratio of interpolated to measured intensities is between 0.996 to 1.00 for diffuse light, between 0.998 to 1.00 for skylight, and between 0.989 to 1.04 for collimated light. These preliminary results suggest that in principle it should be possible to generate the background image from a single plume image taken under field conditions. Figure 4.1b also shows that the diffuse light sources provide more stable results than the collimated light source. This result is expected since the diffuse light source results in insensitivity to minor aberrations in the quality of lenses [Thomson et al., 2008a]. In the following sections, a more rigorous analysis of the factors influencing the accuracy of background interpolation is presented.

\subsubsection{Two Main Factors in the Background Interpolation Analysis: the Span Value and the Plume Size}

The principle of generating an interpolated background from a single plume image has been presented for different light sources in the last section. Since the field based diagnostic would need to use sky-scattered radiation as the light source, further investigation was performed to test the background analysis with skylight under different weather conditions. A preliminary analysis of the background interpolation has shown that the background interpolation error depends strongly on the choice of two parameters: 
the value of span in the Loess function and the width of plume in the image (also called the plume size). The plume size is defined here to be the plume width in the image relative to the total image width. For example, a plume size of $20 \%$ indicates that the plume width in the image is $20 \%$ of the width of the whole image. To evaluate different image configurations, interpolation was performed for different sizes of plumes relative to the image.

To quantify the quality of the interpolation, interpolated intensities in the background sky images were compared to the actual measured intensities. The biases induced by the interpolated backgrounds with respect to different plume sizes were calculated first as an apparent transmissivity:

$$
\tau_{\text {sky }}=I_{\text {sky,fit }} / I_{\text {sky,measured }}
$$

where $I_{\text {sky,fit }}$ is the interpolated sky intensity generated using the Loess fit function and $I_{\text {sky,measured }}$ is the actual measured intensity data. Ideally, the transmissivity would be unity. The total biases introduced by the interpolated sky transmissivity were quantified by integrating $\ln \left(\tau_{\text {sky }}\right)$ over the interpolated region and dividing by that width for comparison. Since the integral of transmissivity across the plume width is proportional to the mass of soot present in a plume, the integration is proportional to an error in the measured soot flux.

To test the background interpolation bias, thirty (30) sky images were collected under clear sky conditions. For each image, six (6) different plume sizes were evaluated. The plume widths were $1 \%, 10 \%, 20 \%, 30 \%, 40 \%$, and $50 \%$ of the image width. For each of these theoretical plume sizes, thirteen (13) different values of span were tested from 0.1 to 0.7 with an increment of 0.05 . As mentioned in Section 4.1.1, the span 
argument of the Loess function is specified as the fraction of the total number of data points in the local fit. The importance of each these points is weighted by their distance from the interpolation point in a cubic function. For example, if the plume size is $20 \%$, a span of 0.1 represents $10 \%$ of actual data points, which are the remaining data points (80\%) after removing $20 \%$ of data from the data set [Cleveland and Loader, 1996].

Shown in Figure 4.2 are the plots of the background interpolation bias for different spans and plume sizes under a clear sky condition. Each data point is the calculated mean of 30 individual measurements. All data shown here have been normalized by the width of the plume in that image to allow direct comparison. The figure illustrates that the bias tends to be less at smaller span values for the tested plume sizes.

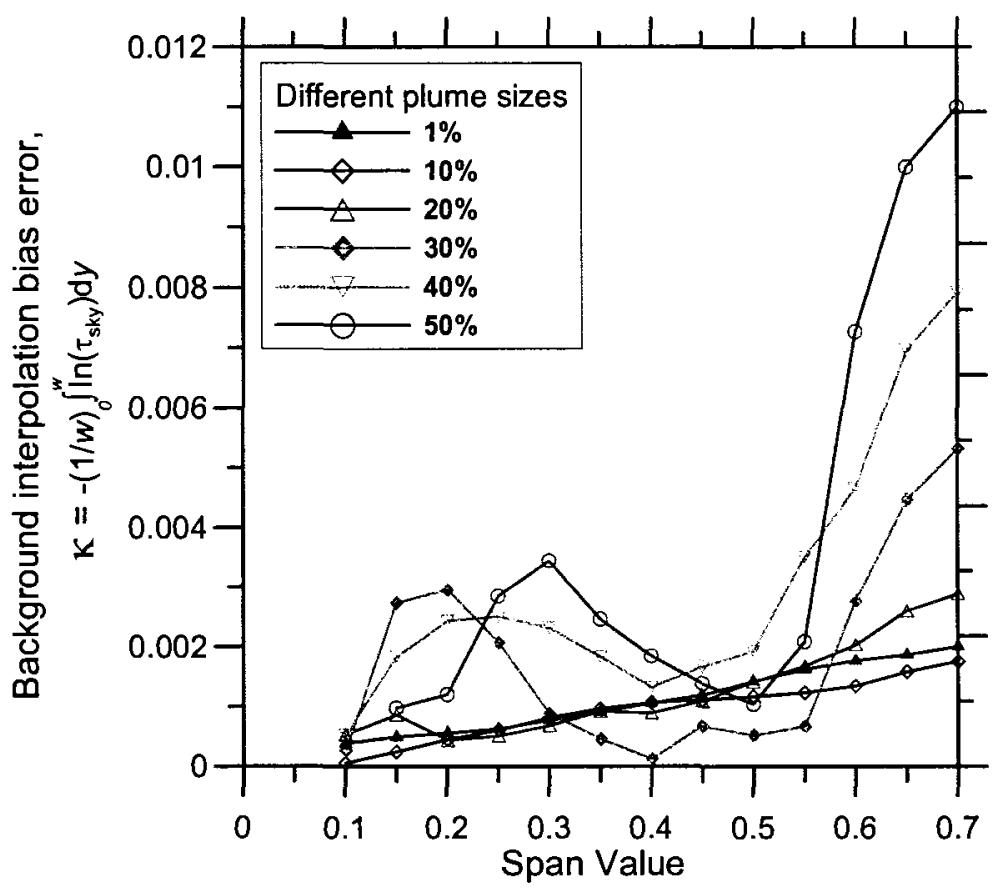

Figure 4.2: Effect of smoothing parameter, span, in Loss function on background interpolation analysis 
Concentrating on the smaller span values, further tests were completed to assess the performance of the algorithm for various plume sizes within the image under different sky conditions. For ease of comparison, the span in the analysis is specified in terms of the number of pixels included in the smoothing algorithm rather than the percentage of pixels included. For example, the sky intensity recorded as a 2D image is 1100 pixels $\mathrm{x}$ 330 pixels which is binned by 10 pixels horizontally leading to a final image width of 110 pixels. For a $20 \%$ plume size, a span of 7 pixels would correspond to a span value in the algorithm of $0.0795(7 /[110 \times(1-20 \%)]=0.0795)$. Pixel spans of 6 to 11 with an increment of 1 pixel (corresponding to the span value as defined in the algorithm of 0.055 to 0.333 ) were evaluated.

The background interpolation biases with respect to different spans in pixels for various sky conditions for plume sizes of $20 \%, 30 \%, 40 \%$, and $50 \%$ are shown in Figure 4.3. Each data point is the calculated mean of 30 individual images with the error bars indicating standard error within a $95 \%$ confidence interval $( \pm 2 \sigma / \sqrt{\mathrm{N}})$ 

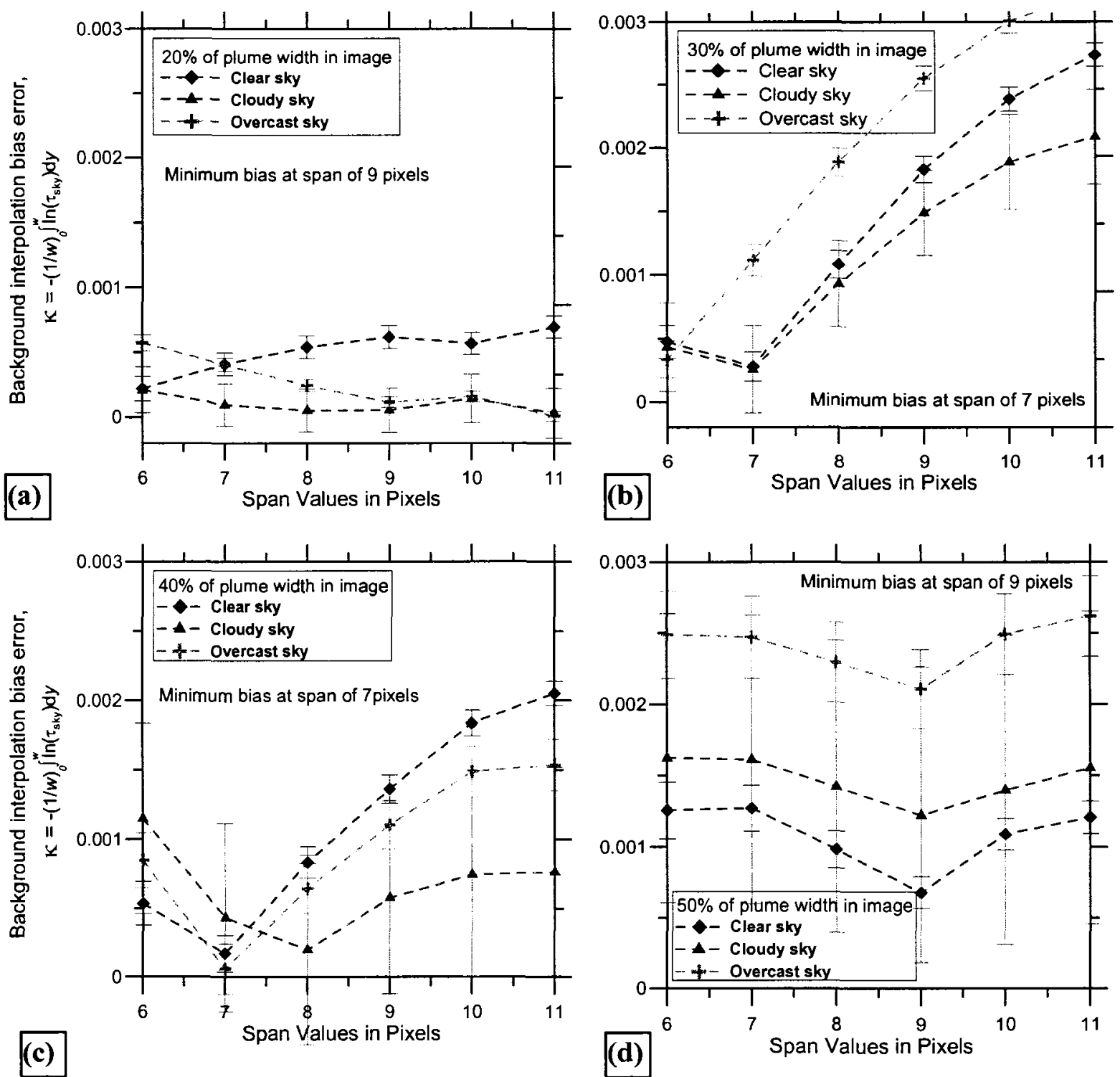

Figure 4.3: Background interpolation bias at different span values under various sky conditions for a) $20 \%$ of plume size, b) $30 \%$ of plume size, c) $40 \%$ of plume size, and d) $50 \%$ of plume size.

As shown in Figures $4.3 \mathrm{a}-\mathrm{d}$, the interpolation bias does not vary significantly with the span for a plume size of $20 \%$ for various sky conditions. However, for plume sizes of $30 \%$ or $40 \%$, the interpolation biases are much more sensitive to the choice of the span. For larger plume sizes, $50 \%$ or $60 \%$ (not shown), the biases are insensitive again with changing span under different sky conditions. Comparing Figures $4.3 \mathrm{~b}$, $\mathrm{c}$, and $\mathrm{d}$ with 
4.3a, the standard errors increase significantly with increasing plume size, especially for the cloudy sky condition. The effect from the plume size is found to be much stronger compared to the effect from different span values. To minimize interpolation errors and to avoid sensitivity to the span value, the optics should be arranged such that the plume size is less than $20 \%$ of the image width. Although there is some variability in the results, a general trend of decreased span with increased plume size was observed.

By observation, optimization of the span setting for Loess interpolation depends on weather conditions, and it is not possible to provide a single guideline for all situations. However, in a practical application of the sky-LOSA technique to measure plumes outdoors, it would be possible to take sky images just before or after actual plume measurements to choose optimal parameters for the Loess interpolation algorithm on a case-by-case basis.

\subsubsection{Quantification of Achievable Background Interpolation Uncertainties}

In the following analysis, the overall uncertainties that could be achieved under a range of sky conditions with optimal span settings as outlined in the previous section were quantified. Figure 4.4 shows bias errors introduced by the interpolation algorithm for different sky conditions, quantified by integrating $\ln \left(\tau_{\text {sky }}\right)$ over the interpolated region and dividing by that width $(\kappa)$, as was done in Figure 4.3. 


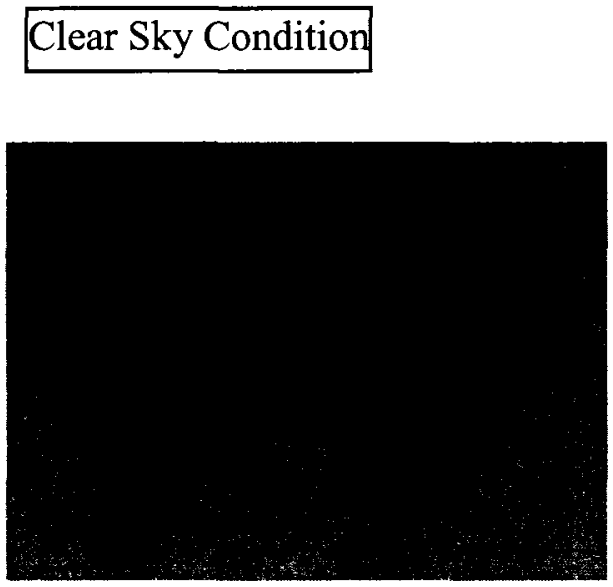

\section{(a)}

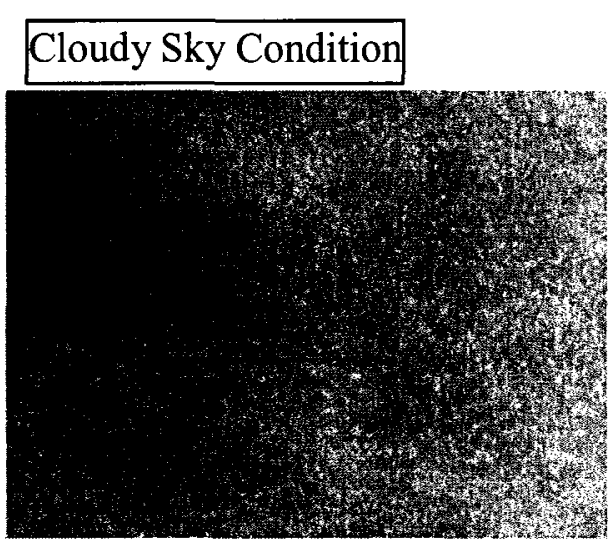

(b)
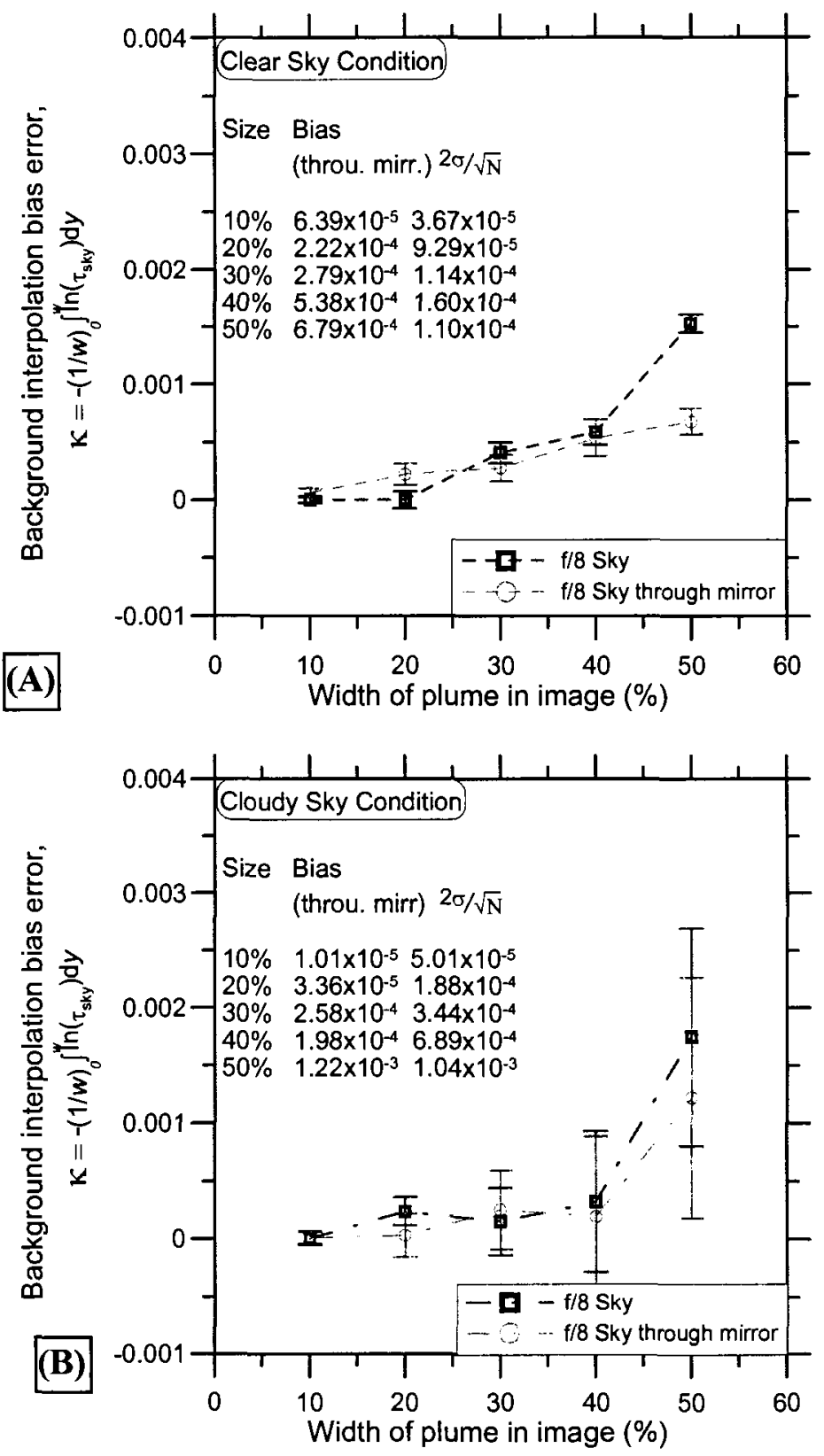


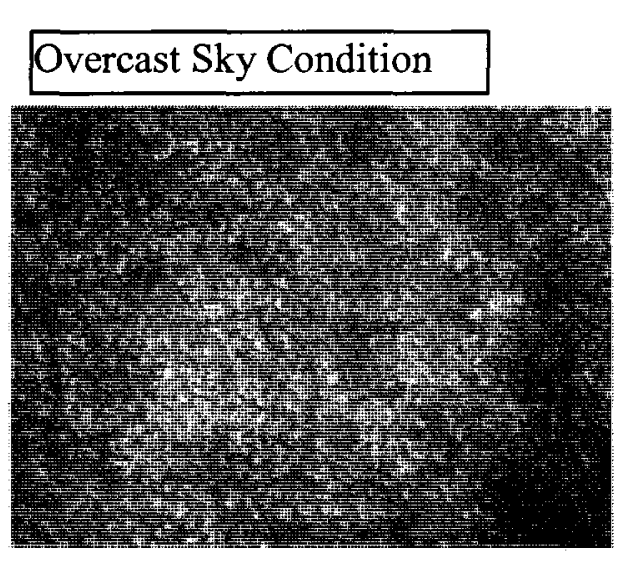

(c)

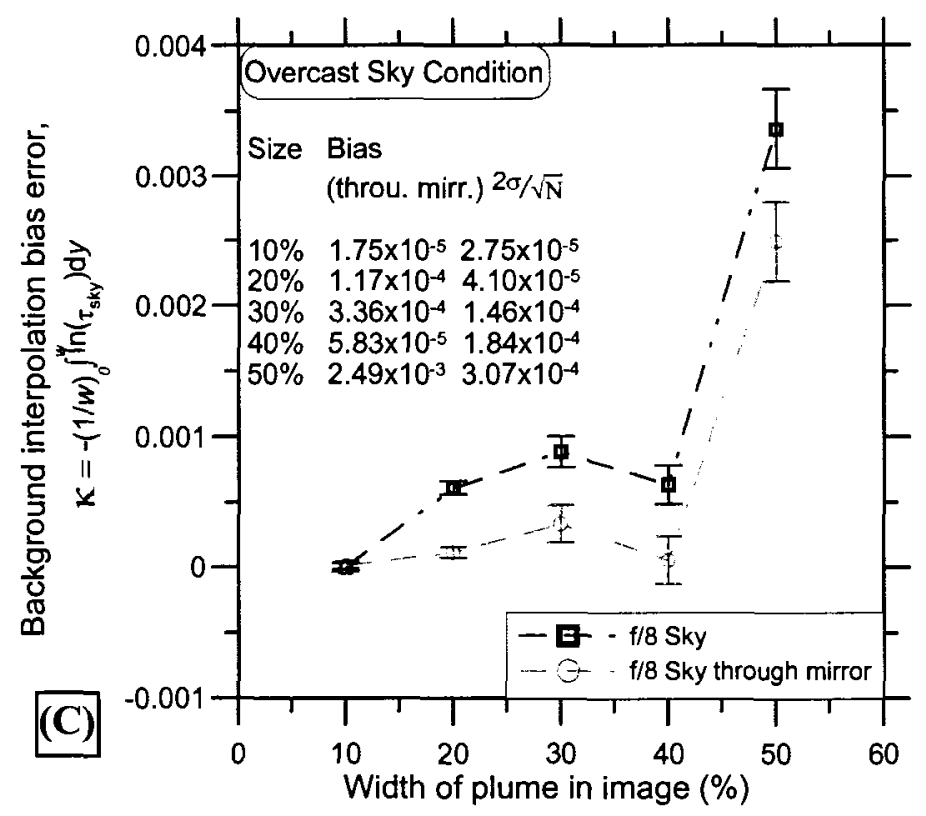

Figure 4.4: a) Clear b) Cloudy c) Overcast sky image. A) B) and C) Background interpolation biases corresponding to each sky condition.

Data points in the figures are calculated means of 30 individual measurements with the error bars indicating standard error within a $95 \%$ confidence interval $( \pm 2 \sigma / \sqrt{\mathrm{N}})$. A table of bias values and the standard errors at different plume sizes is given as an insert in each figure. Each figure shows two curves. The colour of the lines represents whether the images were taken by pointing the CCD directly at the sky (green) or through a mirror (yellow). The mirror was used so that the test plume could remain vertical while ensuring an unobstructed view of the sky as the background in the image. However, in a field measurement, a plume image could be taken by pointing the camera directly to the sky where the plume is issuing from an elevated stack. Tests with and without the mirror were performed to evaluate the effect of the mirror in the measurements. The experimental results show that the background interpolation bias from the data taken 
through the mirror is as good as in those taken directly from the sky, which means the mirror did not influence the measurements.

To be conservative, the interpolation bias under each sky condition should be no larger than the mean bias as indicated in Figure 4.4 plus standard errors of $95 \%$ confidence uncertainty of that mean bias. For the data collected under the three sky conditions presented here, and assuming a $30 \%$ plume size, the biases in $\kappa$ are $3.93 \times 10^{-4}$, $6.02 \times 10^{-4}$, and $4.82 \times 10^{-4}$ for clear, cloudy, and overcast sky conditions, respectively. Comparing various sky conditions, the interpolation biases and the measurement uncertainties under clear sky conditions are the lowest.

Figure 4.5 summarizes the interpolation biases under different sky conditions for the images taken through the mirror (i.e., combines the findings for the case of sky images acquired with a mirror from Figure 4.4 A, B, and C into one figure for comparison). The interpolation biases at $20 \%$ of plume size do not vary significantly with different sky conditions after considering both the measurement uncertainties (standard error at $95 \%$ confidence interval as represented by the error bars). These results suggest that measurements appear possible for clear, cloudy, and overcast sky conditions and the interpolation bias in $\kappa$ is around $2.32 \times 10^{-4}$ if the width of the plume in the image remains less than $20 \%$. Perhaps surprisingly, for plume widths of $40 \%$ or less, there is no consistent advantage of making measurements in one sky condition over another. The uncertainties of the interpolation bias do increase with increasing plume size in the image. 


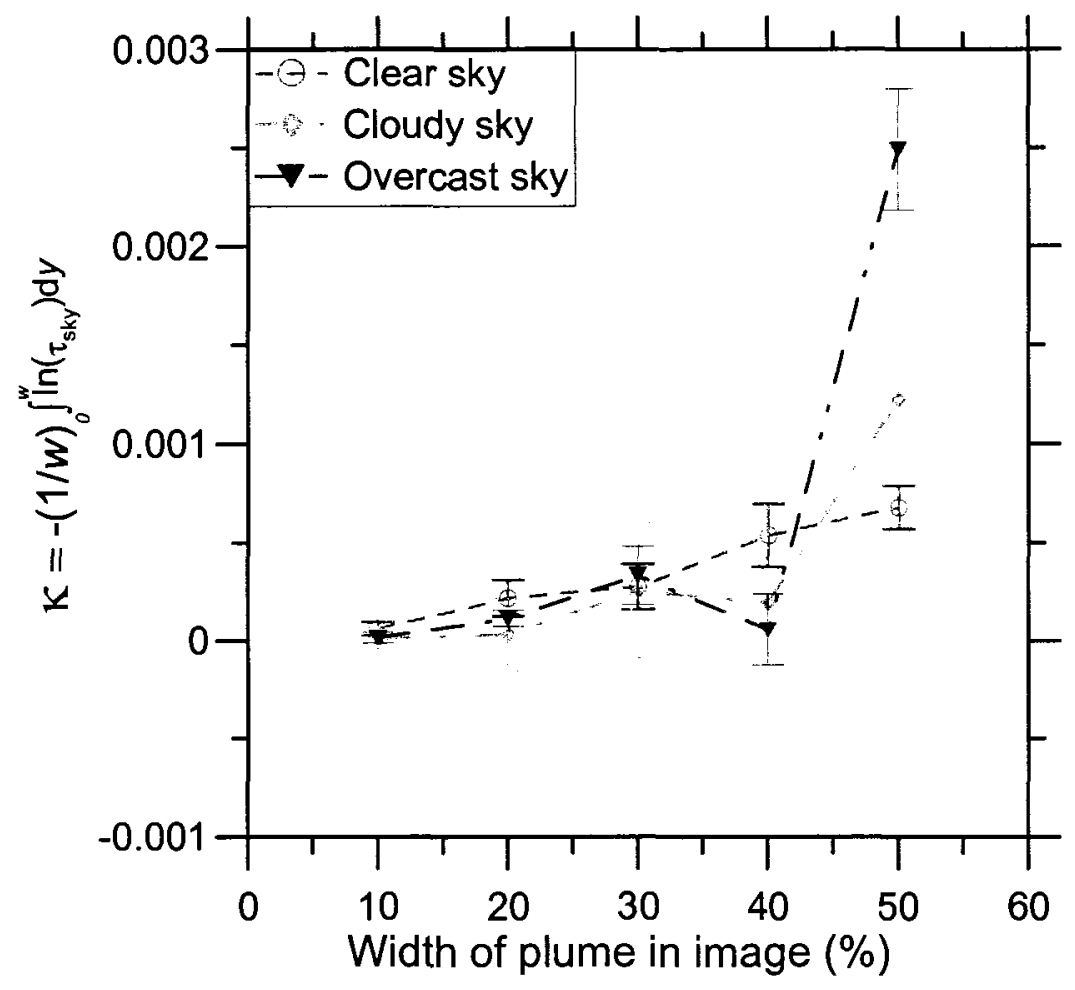

Figure 4.5: Background interpolation bias under three sky conditions through the mirror.

\subsubsection{Null Hypothesis Analysis on the Background Interpolation Bias}

From the results shown in the previous section, it is apparent that the interpolation process leads to a generally positive bias in $\kappa=-\frac{1}{w} \int \ln \left(\tau_{s k y}\right) d y$ as presented in the figures. An extra effort was devoted to understanding whether this apparent positive bias was statistically significant or whether it was indistinguishable from the overall measurement uncertainty. A null hypothesis test combined with 'Student's t Test' was used to complete this investigation.

Ideally, the transmissivity of the sky would be unity, which would lead to $-\int \ln \left(\tau_{s k y}\right) \mathrm{d} y=0$. Thus, in the present case, the null hypothesis, $H_{0}$, was that the 
calculated mean value of $-\int \ln \left(\tau_{s k y}\right) d y, \mu_{0}$, and the anticipated mean value, $\mu=0$, were statistically equivalent.

As outlined in Devore (2004), the test statistic to evaluate the validity of the null hypothesis is:

$$
t=\frac{\bar{x}-\mu_{0}}{S / \sqrt{n}}
$$

where $\bar{x}$ and $S$ are the mean and the standard deviation of the sample data, respectively, $\mu_{0}$ is the expected value, $n$ is the number of sample data.

The calculated $t$ value is then compared with the critical value $t_{\alpha, n-1}$ from the $t$ distribution table. Note that $\alpha$ is called the significance level and is equal to (1-confidence level). If $t \geq t_{\alpha, n-1}$, it means that there is evidence to reject the null hypothesis $H_{0}$ in favor of the alternative hypothesis, $H_{a}$. In other words, there is evidence that the mean is significantly different with the hypothesized value. Table 4.1 summarizes means and standard deviations of sample data at the plume size of $30 \%$ under three sky conditions, test statistic $t$, and critical values of $t_{0.05,29}$ (Devore, 2004).

Table 4.1: Null hypothesis summary table for background interpolation biases under different sky conditions

\begin{tabular}{|c|c|c|c|}
\hline \multirow{2}{*}{} & \multicolumn{3}{|c|}{$-\int \ln \left(\tau_{\text {sky }}\right) d y$} \\
\cline { 2 - 4 } & Clear sky & Cloudy sky & Overcast sky \\
\hline mean $(\bar{x})$ & $1.84 \mathrm{E}-02$ & $1.41 \mathrm{E}-02$ & $2.14 \mathrm{E}-02$ \\
\hline standard deviation $(S)$ & $5.54 \mathrm{E}-03$ & $1.69 \mathrm{E}-02$ & $1.45 \mathrm{E}-02$ \\
\hline Significance level $(\alpha)$ & \multicolumn{3}{|c|}{0.05 or $95 \%$ confidence interval } \\
\hline Number of the sample data $(n)$ & \multicolumn{3}{|c|}{30} \\
\hline Test Statistic Value $t$ & 18.204 & 4.583 & $\mathbf{8 . 0 8 4}$ \\
\hline Critical Value $t_{0.05,29}$ & 1.699 & 1.699 & 1.699 \\
\hline
\end{tabular}


A sample calculation using a significance level of 0.05 (i.e., $95 \%$ confidence level) under overcast sky condition is shown below.

- $\quad \mu$ is the mean $-\int \ln \left(\tau_{\text {sky }}\right) d y$

- Null hypothesis $H_{0}: \mu=0\left(\mu_{0}=0\right)$

- $\quad$ Alternative hypothesis $H_{a}: \mu>0$

- Test Statistic Value: $t=\frac{\bar{x}-\mu_{0}}{S / \sqrt{n}}=\frac{0.0214-0}{0.0145 / \sqrt{30}}=8.084$

- $\quad$ From the table of Critical Values for $t$ Distributions [Devore, 2004]

$$
t_{0.05,29}=1.699
$$

Therefore, since the calculated $t$ value of 8.084 is greater than the critical value of 1.699 (i.e., $t \geq t_{\alpha, n-1}$ ), the null hypothesis $H_{0}$, that is $\mu=0$, is rejected and the alternative hypothesis $H_{a}$, that is $\mu>0$, is in favour.

As shown in Table 4.1 the Test Statistic Values $t$ for different sky conditions are much greater than the critical value $t_{0.05,29}$ for all cases. Thus, the analysis reveals that the background interpolation introduces a positive bias error that, although small, is statistically significant.

This systematic error might come from the application of Loess function in the background interpolation analysis. As explained previously, the Loess function uses a weighted least square regression to locally fit a polynomial on a certain neighbourhood around the point to be evaluated. For example, for the fit at point $\mathrm{x}$, the fit is made using points in a neighbourhood of $\mathrm{x}$, weighted by their distance from $\mathrm{x}$ [Cleveland and Loader, 1996]. In the background interpolation algorithm, the Loess function is used to fit the intensity data to the left and to the right of a region where the intensity data have been 
removed and the locally fitted polynomial is used to generate synthetic intensity data in the plume region. The width of the synthetic data region depends on the plume size in the image. Referring back to Figure $4.1 \mathrm{a}$, the Loess fit closely tracks the intensity data outside the plume region. However this is not always the case within the plume region as evidenced by the interpolation for the collimated light condition (shown in orange and brown) in that same figure.

Figure 4.6 shows the spatial variation of the light intensity of the sky under different sky conditions. While the shapes of the curves vary, they arguably tend to be concave facing downward. This shape could be related to the characteristic of the sky as well as the camera lens itself, and might not best described by a quadratic profile used in Loess function. While this is only a speculation, it would be useful to examine other fit/interpolation schemes or combination of functions in the future. However, although bias error has been found to be statistically significant, it is still very small relative to the uncertainties of individual measurements so that the bias error is not a major limitation of the technique. 


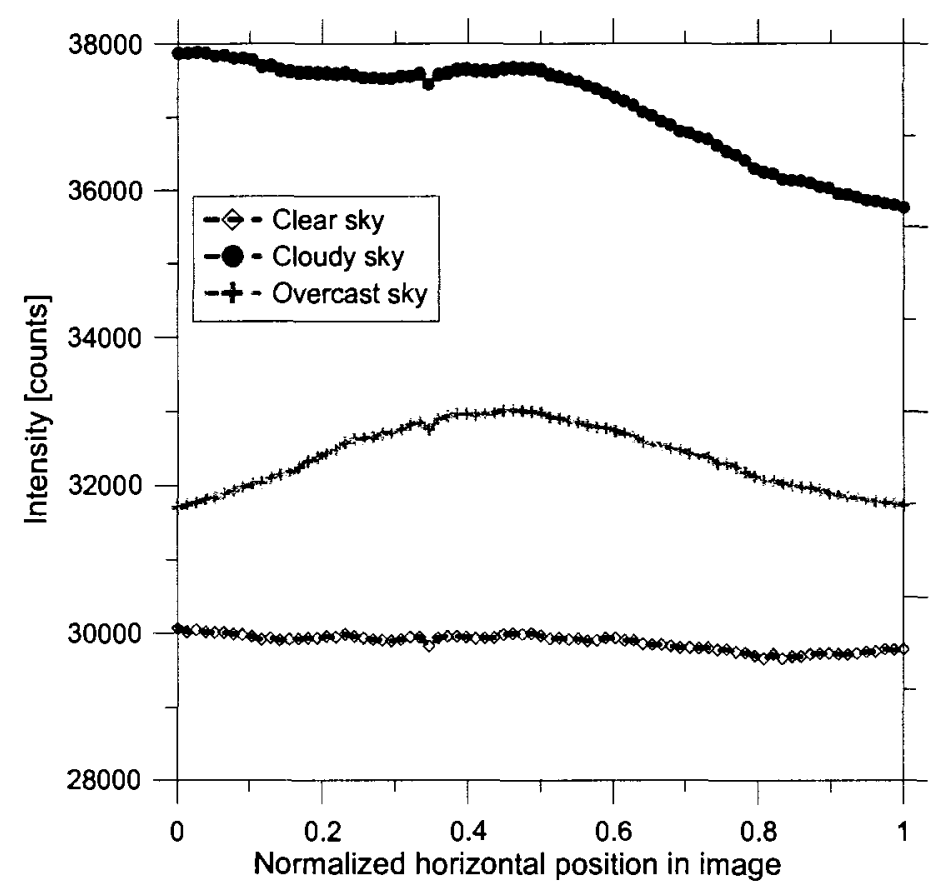

Figure 4.6: Light intensity variation with horizontal position in image for different sky conditions.

\subsubsection{Associated Errors in Soot Emission Rates from Background Interpolation Analysis}

The associated error in soot emission rates contributed by the background interpolation bias was estimated based on Equation 2.18 .

Recall Equation 2.18

$$
\dot{m}_{\text {soot }}=\frac{u \rho_{\text {soot }} \lambda \cdot w}{6 \pi E(m)_{\lambda}\left(1+\rho_{\text {sa }}\right)} \cdot \kappa
$$

The following values can be taken from the experiments or assumed from the literature:

- Soot density $\left(\rho_{\text {soot }}\right)$ is $1.8-1.9 \mathrm{~g} / \mathrm{ml}$ [Rossman and Smith, 1943; Flower and Bowman, 1986; Dobbins, et al., 1994; Wu, et al., 1997];

- The wavelength $(\lambda)$ in the current experiment is $577 \mathrm{~nm}$;

- Refractive index function of soot, $E(m)_{\lambda}$, is 0.258 [Dobbins and Mulholland,1991 and Snelling et al., 1999]; 
- Ratio of scattering to absorption, $\rho_{\mathrm{sa}}$, is 0.2 [Johnson and Thomson, 2005];

- Plume is two (2) meters wide; and

- Plume velocity $(u)$ is $12.5 \mathrm{~km} / \mathrm{h}$ (corresponding to a typical average wind speed for locations in Alberta).

From Figure 4.4 A) in Section 4.1.3, the background interpolation bias for a clear sky condition is $3.93 \times 10^{4}$ assuming $30 \%$ of plume size, corresponding to a soot emission rate of

$$
\begin{aligned}
\dot{m}_{\text {soot }} & =\frac{u \rho_{\text {soot }} \lambda \cdot w}{6 \pi E(m)_{\lambda}\left(1+\rho_{\text {sa }}\right)} \cdot \kappa \\
& =\frac{12.5 \frac{\mathrm{km}}{\mathrm{h}} \times 1.8 \frac{\mathrm{g}}{\mathrm{ml}} \times 577 \mathrm{~nm} \times 2 \mathrm{~m}}{6 \pi \times 0.258 \times(1+0.2)} \times\left(3.93 \times 10^{-4}\right) \\
& =0.48 \frac{\mathrm{mg}}{\mathrm{s}}
\end{aligned}
$$

Compared to an estimated nominal soot emission rate from solution gas flares of $4.2 \mathrm{mg} / \mathrm{s}$ [Johnson and Thomson, 2005], the uncertainty due to the background interpolation is estimated as follow.

$$
\frac{0.48 \frac{\mathrm{mg}}{\mathrm{s}}}{4.2 \frac{\mathrm{mg}}{\mathrm{s}}}=0.11=11 \%
$$

The associated errors in soot emission rates contributed by the background interpolation bias are estimated, based on Equation 2.18 , to be $0.48 \mathrm{mg} / \mathrm{s}, 0.74 \mathrm{mg} / \mathrm{s}$, and $0.59 \mathrm{mg} / \mathrm{s}$ for clear, cloudy, and overcast sky conditions, respectively for $30 \%$ of plume size, and $0.29 \mathrm{mg} / \mathrm{s}$ under all sky conditions for a $20 \%$ of plume size. Compared to the estimated soot emission rate of $4.2 \mathrm{mg} / \mathrm{s}$ from solution gas flares, these uncertainties in background interpolation would equate to an uncertainty in the measured soot mass flow 
rate of $11 \%, 17 \%$, and $14 \%$ for clear, cloudy, and overcast sky conditions at $30 \%$ of plume size and $6.8 \%$ at $20 \%$ of plume size. It should be noted that these uncertainties are relative to the target soot yield rate of $4.2 \mathrm{mg} / \mathrm{s}$ and the relative uncertainties would decrease if soot emission rates were greater than $4.2 \mathrm{mg} / \mathrm{s}$.

\subsection{Additional sources of uncertainty in transmissivity measurements}

\subsubsection{Nearest Limit of Object Distance and Associated Error}

The magnification of a camera system is calibrated in the lab by imaging an object of known size. The scaling factor can be precisely calculated by counting the number of corresponding pixels seen on the image. However, in a field application, a plume to be measured could be hundreds of meters away where it would clearly not be practical to put a known size target next to the plume. Therefore, a simple calibration procedure is proposed that includes the use of a commercially available laser range finder (Advantage Range Finder with Compass/Inclino Meter LA 1095-020). A step-by-step camera system calibration procedure and simulation results are presented in more detail in Appendix A.

For the specific case of the present CCD array, which has a chip size of $7.8 \mu \mathrm{m} x$ $7.8 \mu \mathrm{m}$ coupled to Micro-Nikkor $105 \mathrm{~mm}$ lens, and with a desired plume size in the image of $20 \%$, Figure 4.7 summarizes the recommended distance of the observer (or CCD) from the plume as a function of the plume width (diameter) for different focal length lenses. Figure 4.8 summarizes the uncertainty of soot yield rate measurement that can be attributed to uncertainty in the spatial calibration for the same focal length lenses. 


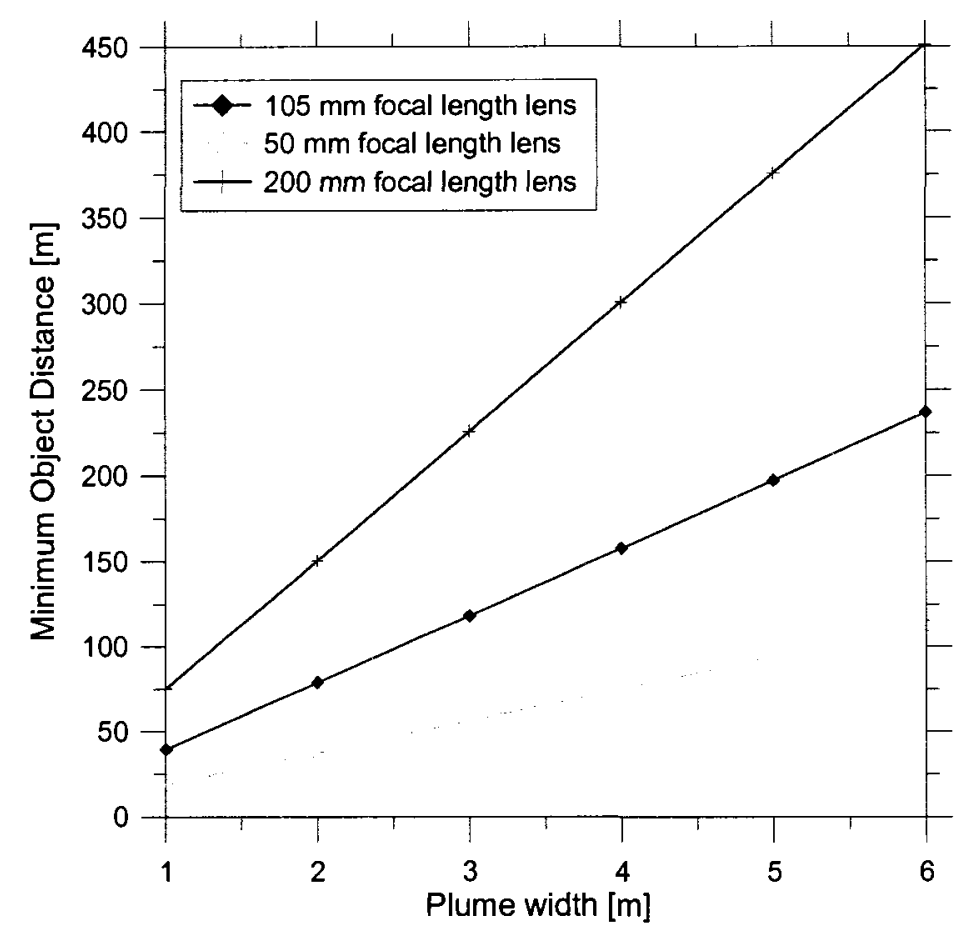

Figure 4.7: Recommended distances of the observer from a plume for $20 \%$ plume size.

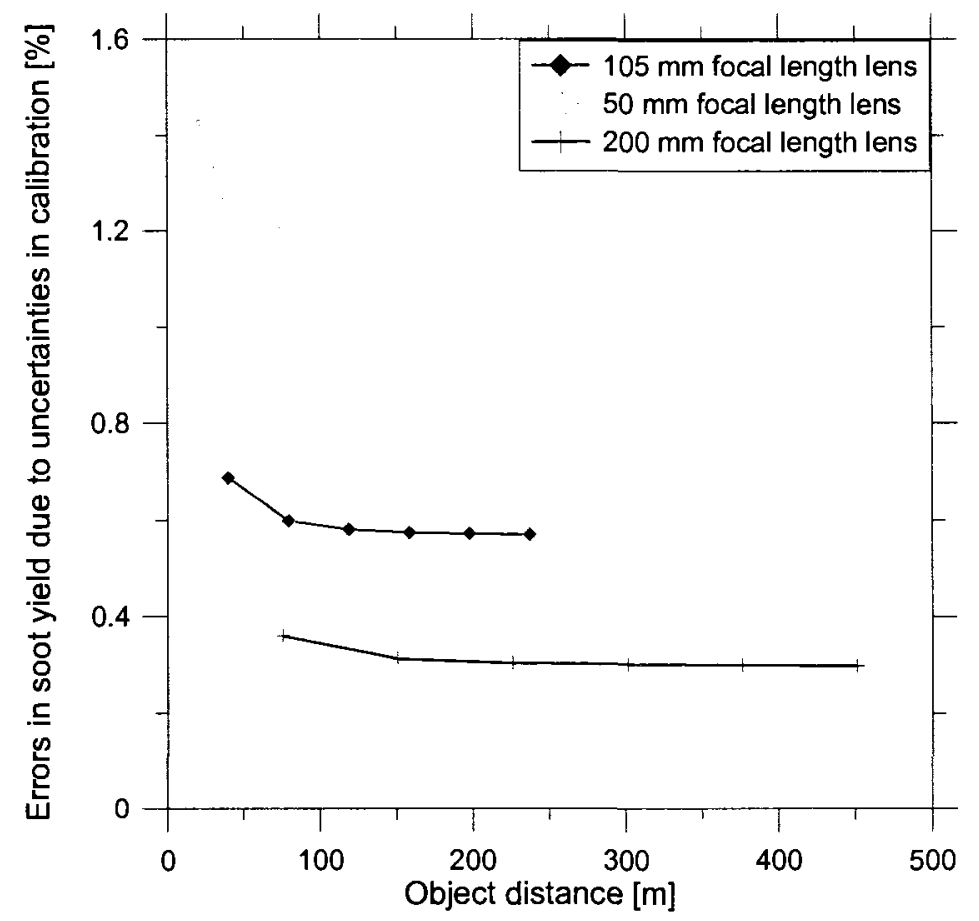

Figure 4.8: Associated errors in soot yield rates due to uncertainties in calibration for $20 \%$ plume size. 
As shown in Figure 4.7, the minimum object distance to maintain 20\% plume width in the image increases linearly with increasing the plume width. The uncertainty in the soot yield rate that is attributed to uncertainty in the spatial calibration decreases with increasing distance of the lens from the plume, since the uncertainty of the laser range finder is a fixed quantity. For a $1 \mathrm{~m}$ wide plume, the camera should be positioned no less than $39.5 \mathrm{~m}$ away from the plume in order to keep the plume width less than $20 \%$ of the image for the $105 \mathrm{~mm}$ focal length lens used in the experiments. In this case, the spatial calibration uncertainty in the current optical system would contribute a relative uncertainty in soot yield rate of $0.68 \%$ for the same lens as shown in Figure 4.8 . For a longer focal length lens (i.e., $200 \mathrm{~mm}$ in Figure 4.7), the object distance should be longer than that in $105 \mathrm{~mm}$ focal length lens to keep the same size of the plume in the image and the uncertainty contributed from calibration decreases while using longer focal length lens as shown in Figure 4.8. For the plume size of 30\%, simulation results of minimum object distances and associated errors in soot emission rates for the lens with focal length of $105 \mathrm{~mm}$ are shown in Figure A.6 and A.7 in Appendix A.

A useful artifact of using skylight as the light source for attenuation measurements is that the distance from the detector to the light source is essentially fixed at infinity and thus the solid angle of sky sampled in the image is fixed by the focal length of the lens. In the context of background interpolation, the results presented here are valid whether the plume measured is 1 meter in diameter, 40 meters away or 4 meters in diameter, 160 meters away. Since the distance to the sky is essentially fixed, the imaging characteristic of the sky will be the same for both plumes. A caveat to this observation is that a 4 meter diameter plume with the same soot emission rate as a 1 meter plume will be more 
transparent and thus more difficult to detect. Nonetheless, the absolute error introduced by the background interpolation will be the same for both cases. A second caveat is that changing the focal length of the lens will change the solid angle of sky sampled and thus the background interpolation should be re-evaluated for each focal length of lens used.

\subsection{Summary of background interpolation sensitivity and uncertainty}

Two parameters in the background interpolation, the value of span in the Loess function and the plume size, have been investigated. For different weather conditions, there was no significant effect of changing the span value in background interpolation analysis when the plume size was less than $20 \%$ of the image width. The effect of plume size in the image (i.e., size of the interpolation region) was found to be much stronger compared to the effect of changing span values.

The results show that background intensity in the plume region can be accurately interpolated from unattenuated intensity data outside of the region. A clear sky condition is preferred for plume transmissivity measurements in the field if the width of the plume in the image is $30 \%$ of image width. However, measurements appear possible for any condition if the plume size is less than $20 \%$. Compared to the estimated nominal soot emission rate of a solution gas flare of $4.2 \mathrm{mg} / \mathrm{s}$, the background interpolation biases represent uncertainties of $11 \%, 17 \%$, and $14 \%$ for clear, cloudy, and overcast sky conditions, respectively, at $30 \%$ of plume size and $6.8 \%$ for all these three sky conditions at $20 \%$ of plume size. These uncertainties are relative to the target soot yield rate of $4.2 \mathrm{mg} / \mathrm{s}$ and the relative uncertainties would decrease if soot emission rates are greater than $4.2 \mathrm{mg} / \mathrm{s}$. 


\section{Results: Sensitivity and Uncertainty of Plume Measurements}

\subsection{Target Soot Emission Rate}

The primary motivation for this work was to develop a diagnostic capable of measuring soot emissions from solution gas flares typical of those found in the Province of Alberta. Based on a detailed analysis of 1999 data from the Alberta Energy and Utilities Board (AEUB), Johnson et al. (2001) estimated that there were 4499 solution gas flares operating in Alberta. Reported flared volumes were approximately log-normally distributed and varied significantly among sites with a mean of $316,200 \mathrm{~m}^{3} /$ year, median of $60,300 \mathrm{~m}^{3} /$ year, and a range of 500 to $30,000,000 \mathrm{~m}^{3} /$ year. Most solution gas flares, however, (95\%) processed less than $1,000,000 \mathrm{~m}^{3} /$ year.

Other types of flares are also common in the upstream oil and gas industry, such as well-test flares and emergency flares. However these flares generally process much larger volumes of gas. Smaller solution gas flares, which would be expected to produce comparatively lower amounts of soot, could thus be considered as the limiting case for required sensitivity in a soot flux diagnostic.

Development of accurate soot emission guidelines for solution gas flares is an area of ongoing research (e.g. Canteenwalla, 2007). Based on limited data in the literature for soot emissions from vertical diffusion flames burning methane and propane, Johnson and Thomson (2005) estimated an approximate soot emission rate of $4.2 \mathrm{mg} / \mathrm{s}$ from an 
average sized solution gas flare in Alberta. This value is used here as a target rate for assessing the capabilities of the soot diagnostic being developed. For a given set of environmental conditions, measurements would become easier if the emission rate exceeded the target of $4.2 \mathrm{mg} / \mathrm{s}$.

As mentioned in Section 2.3.2, the term $\int \ln \left(\tau_{\lambda}(y)\right) d y$ is an integration of local transmissivity over the width of the plume, which determines the soot concentration. Since the plume width varies under different test conditions, as shown previously in Equation 2.17 , it is helpful to normalize $\int \ln \left(\tau_{\lambda}(y)\right) d y$ by the width of the plume to define a parameter $\kappa$, such that $\kappa=-(1 / w) \int \ln \left(\tau_{\lambda}(y)\right) d y$. The term $\kappa$ can then be used to evaluate the performance of the sky-LOSA diagnostic independent of the physical size of the plume being measured. Recalling Equation 2.18,

$$
\dot{m}_{\mathrm{soot}}=\frac{u \rho_{\mathrm{soot}} \lambda \cdot w}{6 \pi E(m)_{\lambda}\left(1+\rho_{\mathrm{sa}}\right)} \cdot \kappa
$$

for the target soot flux of $4.2 \mathrm{mg} / \mathrm{s}$ from a solution gas flare, a target value of $\kappa$ can be calculated that needs to be achieved by the prototype diagnostic.

Figure 5.1 shows calculated values of $\kappa$ for various emission rates of soot into 1, 2, or $3 \mathrm{~m}$ diameter plumes moving at speed of $12.5 \mathrm{~km} / \mathrm{h}$ (corresponding to a typical average wind speed for Alberta). For the target mass flux of $4.2 \mathrm{mg} / \mathrm{s}$ (indicated by the dashed vertical line) emitted in a $2 \mathrm{~m}$ diameter plume, the corresponding value of $\kappa$ is 0.0034. If that same mass flux is confined within a 1 or $3 \mathrm{~m}$ diameter plume, then the target value of $\kappa$ is 0.0068 or 0.0023 . In the following sections, experimental results are presented in an attempt to quantify the achievable sensitivity of the sky-LOSA technique 
and compare this with the required values of $\kappa$ necessary to measure emissions of solution gas flares.

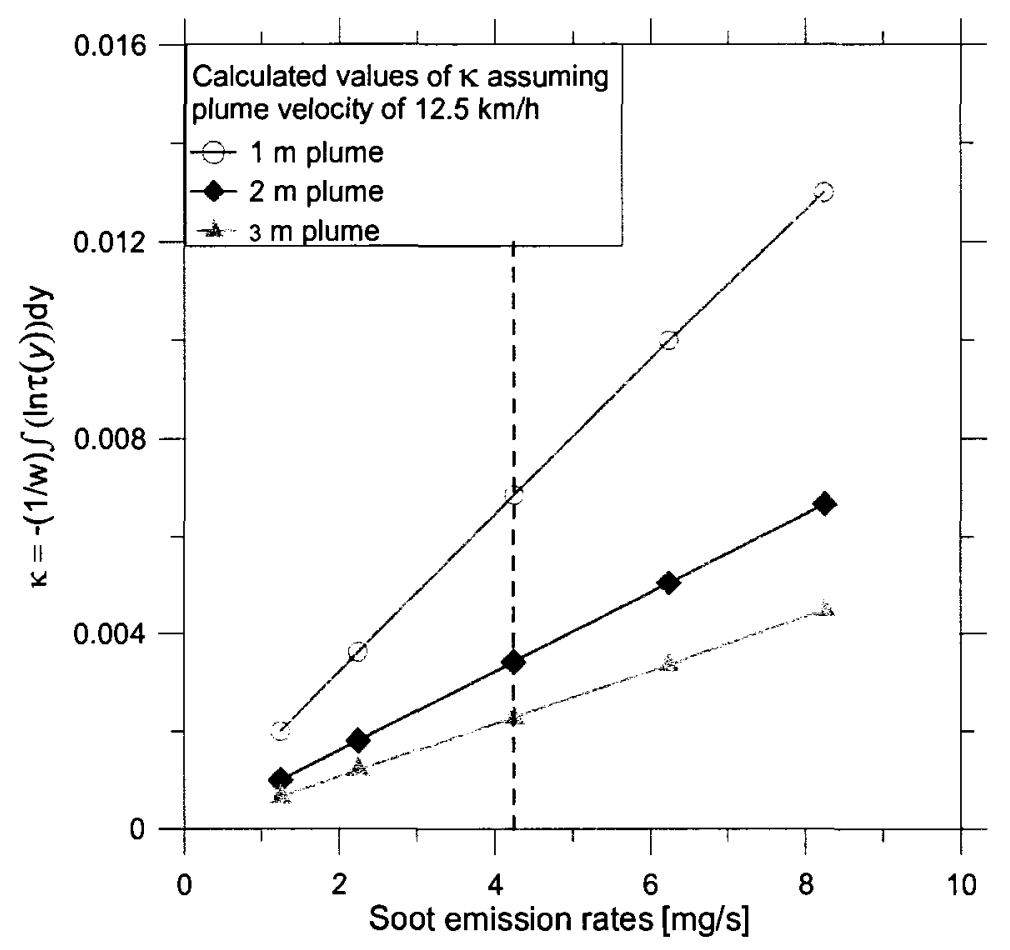

Figure 5.1: $\kappa=-\frac{1}{w} \int \ln \left(\tau_{\lambda}(y)\right) d y$ as a function of soot yield rate. Dashed line indicates target $4.2 \mathrm{mg} / \mathrm{s}$ soot emission rate estimated for solution gas flares.

\subsection{Results: Artificial Test Plume Experiments}

During initial development of the LOSA diagnostic, artificial test plumes were generated for use in the experiments by thermophoretically depositing flame-generated soot onto microscope slides. A detailed description of the artificial test plumes can be found in Section 3.4.2. Seven test plumes were generated with minimum transmissivities ranging from 0.4 to 0.983 . 
To test the detectable limits of the transmissivity measurement, experimental data were gathered for three light sources: collimated light from an arc-lamp, diffuse light from and arc-lap coupled with an integrating sphere, and sky-scattered solar radiation. For each light source, 30 plume attenuating images were gathered for each sample plume. Two reference images (background and dark) were also collected for each experiment in the lab when using the collimated and diffuse light sources. The data sets collected under the different light sources were evaluated and compared by using the 3-image and 1image methods (refer to Section 2.3.3). For the 1-image method, the background intensity was generated from the intensity data to the left and to the right of the plume in transmission image using the Loess fit function as discussed in Section 4.1.

Figure 5.2 shows comparison plots of $\kappa$ values for the artificial test plume with the highest transmissivity $\left(\tau_{\lambda}=0.983\right)$. Data for the 1 -image and 3 -image methods is shown as a function of distance above an arbitrary point of the plume. The data points on the graph are the calculated means of the 30 individual measurements with uncertainty bars indicating the $95 \%$ confidence interval $( \pm 2 \sigma)$. For both diffuse light and sky-scattered light sources, the agreement between the 1-image and 3-image methodologies appears well within experimental uncertainty. There is notable additional scatter in the data for the 1-image method with collimated light. As expected, this suggests that the use of diffuse light as a source for transmissivity measurements should be advantageous primarily due to the resulting insensitivity to minor aberrations in the quality of lenses [Thomson et al., 2008a].

In Figure 5.2b, the 1-image collimated data is omitted and the vertical scale adjusted to highlight differences in the data. From this figure we can see that the skylight 
measurements agree well with the 3-image diffuse light measurements and are also close to the 3-image collimated results. These data suggest that the 1-image method with skyscattered solar radiation as light source is a promising method for plume transmissivity measurements in a field setting.
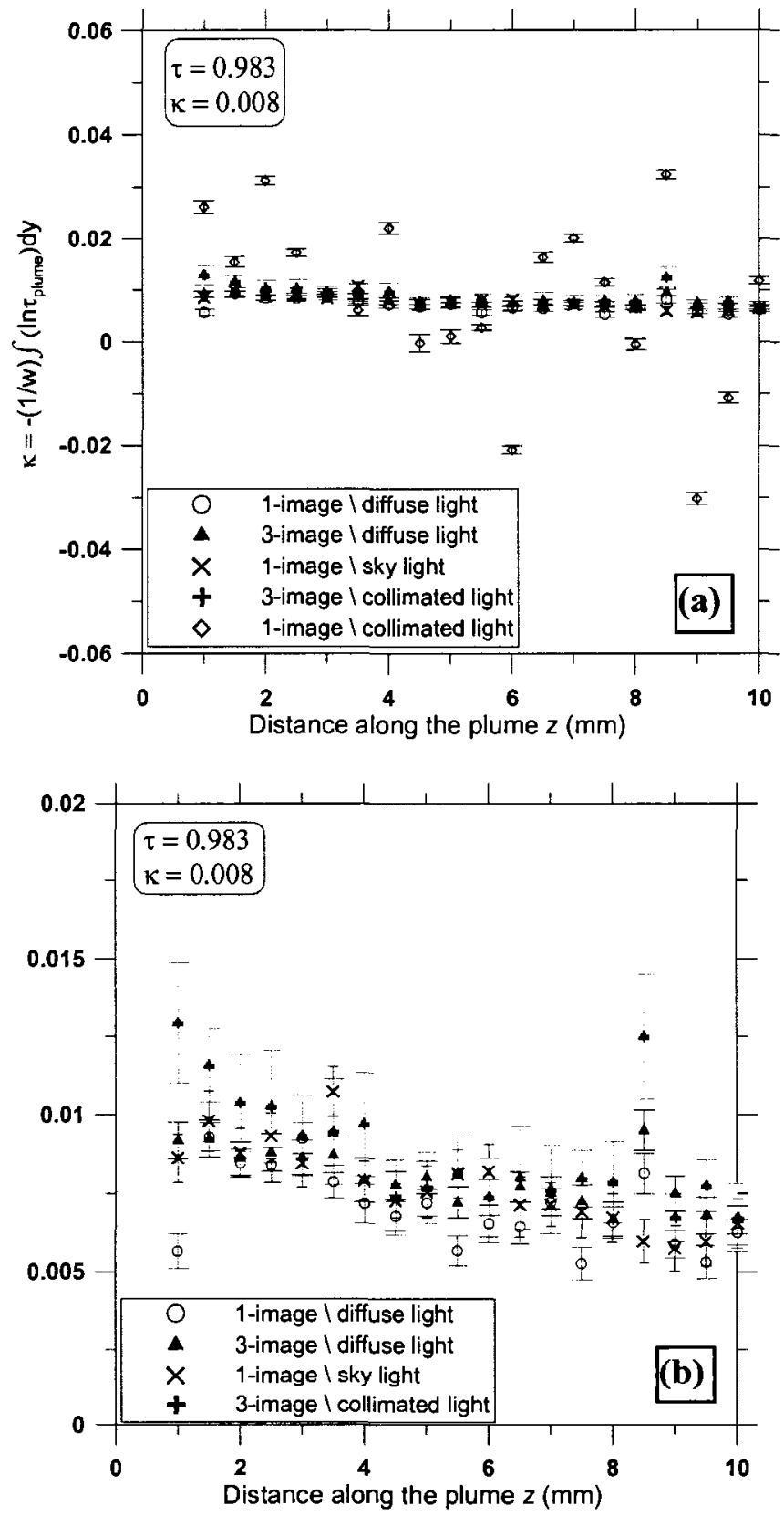

Figure 5.2: a) comparison of 1- and 3-image LOSA techniques for $\kappa$ with 3 light sources b) Zoomed-in plot without 1-image, collimated light data for artificial test plumes. 
To quantify the difference between the 1-image method with skylight and the 1- and 3-image methods with diffuse light from an arc lamp, we further evaluated the averages of 30 individual measurements of each of the seven (7) sample plumes at multiple heights along the plume, using each light source. Figure 5.3 shows the \% uncertainty in the measurements, where the 3-image, diffuse-LOSA technique has been taken as a standard.

As expected, the uncertainty increases sharply as the transmissivity of the samples approaches unity. The results suggest that with the current setup, comparative uncertainties of less than $40 \%$ are achievable for plume transmissivities around 0.983 corresponding to $\kappa=0.008$. However, this limiting $\kappa$ value might be an artifact of the synthetic plume rather than an absolute limit of the system. Improvements to this upper limit on transmissivity might be possible with an improvement to the synthetic plumes. For example, reflections from the microscope slides (which are purely an artifact of our synthetic sample) could be a source of noise in the results. Therefore a plume generated by a lab-controlled burner was considered in subsequent tests. 


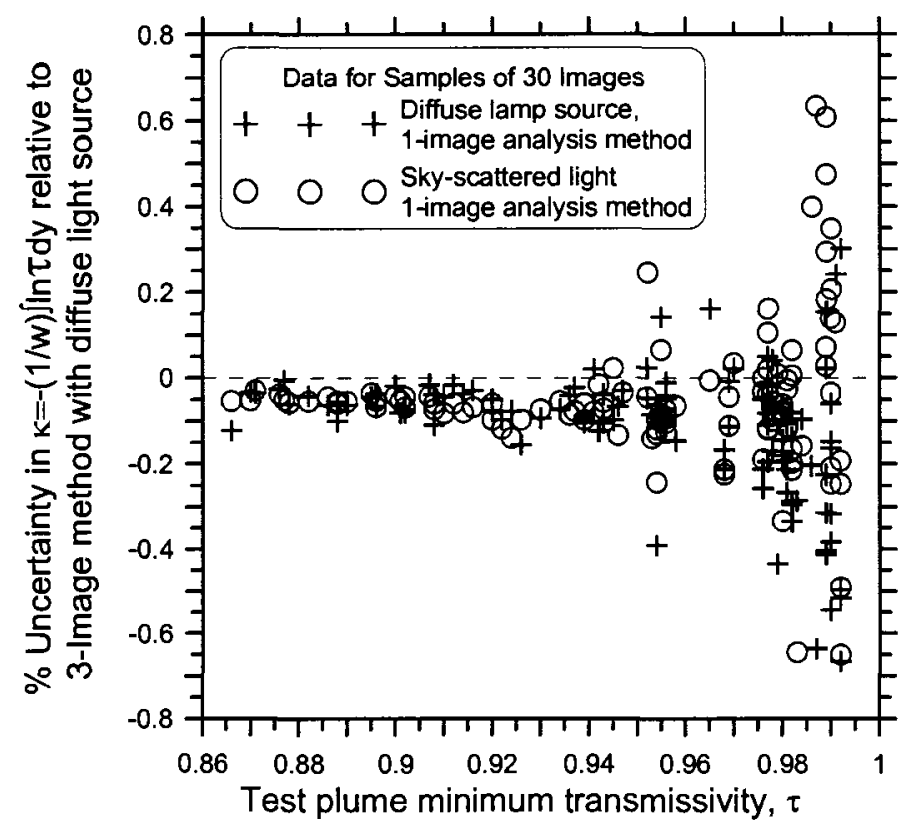

(a)

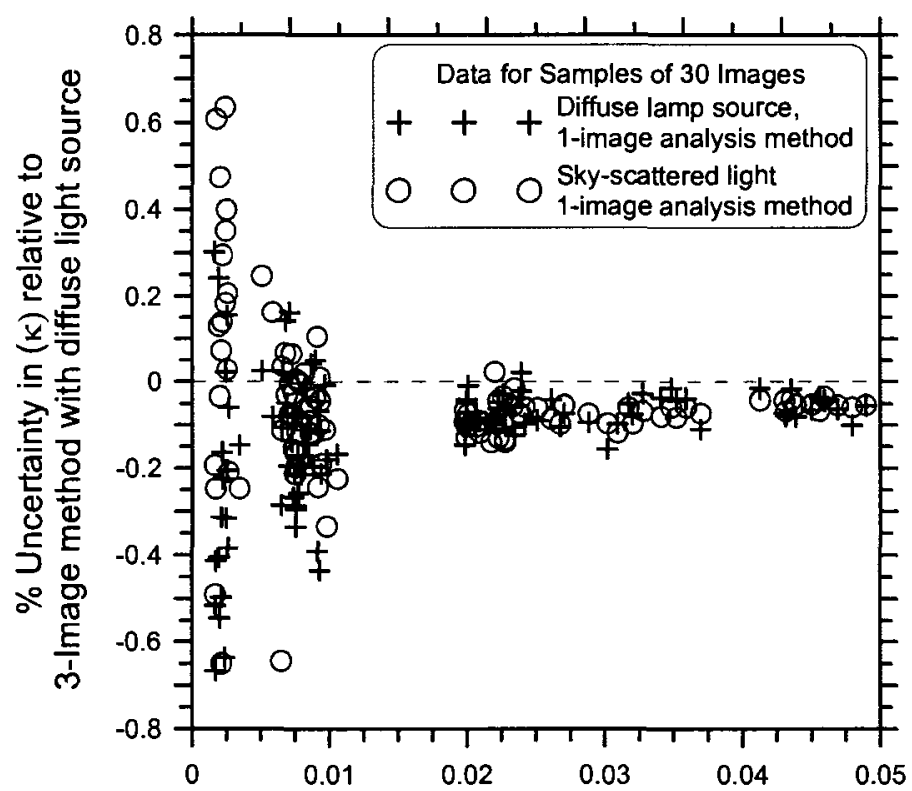

(b)

(к) across test plume determined using

3-image method with diffuse light source

Figure 5.3: Measured uncertainties in $\kappa$ for test plumes using sky-LOSA and 1image diffuse LOSA with 3-image diffuse LOSA as a standard a) uncertainty plotted vs. plume transmissivity and b) uncertainty plotted vs. $\kappa$

\subsection{Results: Unconfined Plume Experiments}

In a final phase of this project, measurements were attempted on unconfined soot plumes issuing into the open atmosphere. Creation of stable, repeatable plumes for testing was a 
challenge that was eventually met by using the inverted lab-controlled burner described in Section 3.4.3. Experiments were conducted to directly compare outdoor sky-scattered solar radiation measurements with in-lab diffuse-LOSA measurements for equivalent test conditions. In this manner, the ultimate accuracy and sensitivity limits of the sky-LOSA approach were investigated.

Experimental data were collected by taking thirty (30) plume attenuating images for each test condition (listed in Table 3.2) using both in-lab diffuse light and skylight under clear sky, cloudy sky, and overcast sky conditions. For the diffuse-LOSA measurements thirty (30) images were also taken of the light source without the plume present at each condition. A reference dark image was collected at the end of the each experiment. Plume transmissivities were calculated using the 3-image routine for diffuse-LOSA and 1-image routine for sky-LOSA measurements.

For the 1-image method, the background intensity was generated from the intensity data to the left and to the right of the plume using the Loess fit function. It should be noted that while generating the background intensity, the width of the plume for interpolation was determined 'by eye'. This was more critical for low light attenuating plumes and/or on windy days since the plume edges were not well defined in these images. In the present experiments, the burner tip was $13.2 \%$ of the image width. The plume sizes were estimated to $15 \%$ to $22 \%$ of the image width depending on the amount of wind-induced movement. Although it would be possible to use edge detection algorithms to automatically estimate the width of the plume, it was not obvious that these routines would improve results. Theoretically, overestimating the plume width would not alter the final results since transmissivities outside the plume should be unity. However, 
to minimize bias due to interpolation uncertainty, it would be preferable to avoid overestimation of the plume width as much as possible.

Figure 5.4 shows comparison plots of $\kappa$ values at equivalent test conditions for 3image diffuse-LOSA measurements and 1-image sky-LOSA measurements under clear, cloudy, and overcast sky conditions. The vertical bars on the figure are the calculated means of 30 individual measurements with error bars indicating standard error within $95 \%$ confidence intervals $( \pm 2 \sigma / \sqrt{N})$.

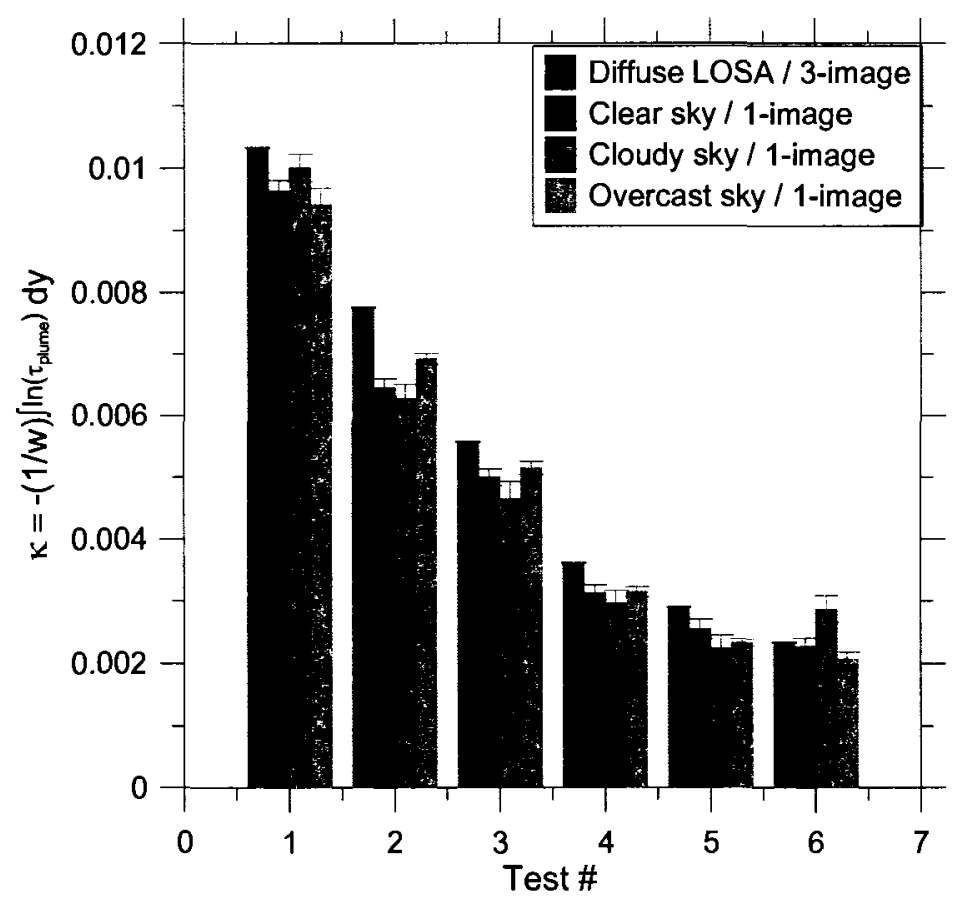

Figure 5.4: Comparison of 3-image diffuse LOSA and 1-image sky-LOSA under different sky conditions at the same test conditions after considering the biases in background interpolation analysis.

Previously it was shown that there are positive biases in the background interpolations (denoted as bias) with associated standard errors (denoted as $\delta_{\text {bias }}$ ) for each of the different sky conditions. These artificial biases should be subtracted from the 
means of plume measurement data. Therefore, data columns from sky-LOSA measurements in Figure 5.4 are calculated as the mean of $\kappa$ from transmissivity measurements minus calculated interpolation biases from the background analysis for each respective sky condition. The standard error in sky-LOSA measurements is calculated by combining the contribution due to the uncertainty in plume transmissivity measurements (denoted as $\delta_{\text {trans }}$ ) and in the background analysis in quadrature $\left(\delta=\sqrt{\left(\delta_{\text {trans. }}\right)^{2}+\left(\delta_{\text {bias }}\right)^{2}}\right)$.

Overall, the 1-image skylight measurements show reasonably good agreement with the 3-image diffuse light measurements. However, the mean of $\kappa$ values from skyLOSA are lower than reference values from 3-image diffuse-LOSA measurements for most test conditions. Statistical testing revealed that this systematic bias was statistically significant, and is partially attributable to the slight bias in background interpolation. Another possible reason for this inconsistency is the differences in ambient temperature when the burner was subjected to cold temperatures (via the open garage door) for skyLOSA experiments relative to room temperature operation during reference diffuse LOSA experiments. This temperature difference may have influenced the temperature at the exhaust outlet of the burner and affected conditions in the plume. A cooler wall temperature of the exhaust tube in sky-LOSA measurement would tend to draw more soot from the exhaust stream due to the thermophoretic effect. The influence of the ambient temperature on the repeatability of the plume generated by this burner needs to be investigated in the future. Ideally diffuse- and sky-LOSA measurements should run simultaneously under identical environmental conditions for future investigation. However, this was not logistically possible during the present experiment. 
It is observed from Figure 5.4 that the standard error (uncertainty of $\kappa$ ) does not vary significantly with different test conditions for each sky condition. The average uncertainties of $\kappa$ are $2.97 \times 10^{-4}, 3.56 \times 10^{-4}$, and $2.90 \times 10^{-4}$ for clear, cloudy, and overcast sky conditions. Test condition 6 represents the minimum soot loading that was measured. In test condition 6 , the minimum transmissivity through the centre of the plume was 0.995 corresponding to a $\kappa$ value of 0.0023 . Lower soot loadings were not attempted because it became impossible to observe the location of the plume, even though the background interpolation bias analysis suggested that higher minimum transmissivities should be possible.

Based on this observed minimum transmissivity limit of 0.995 corresponding to a $\kappa$ value of 0.0023 , Figure 5.5 shows the theoretical minimum detectable mass flow rate of soot for a $2 \mathrm{~m}$ wide plume as a function of plume velocity. Soot emission rates corresponding to the regions above the dashed lines are detectable for each sky condition. The detectable limits deteriorate (i.e., increase) as plume velocity increases. At a typical average wind speed of $12.5 \mathrm{~km} / \mathrm{h}$ for locations in Alberta, the theoretical minimum mass flow rate of soot that could be detected in a $2 \mathrm{~m}$ wide plume is $3.5 \mathrm{mg} / \mathrm{s}, 4.4 \mathrm{mg} / \mathrm{s}$, and $3.4 \mathrm{mg} / \mathrm{s}$ for clear, cloudy, and overcast sky condition, respectively. 


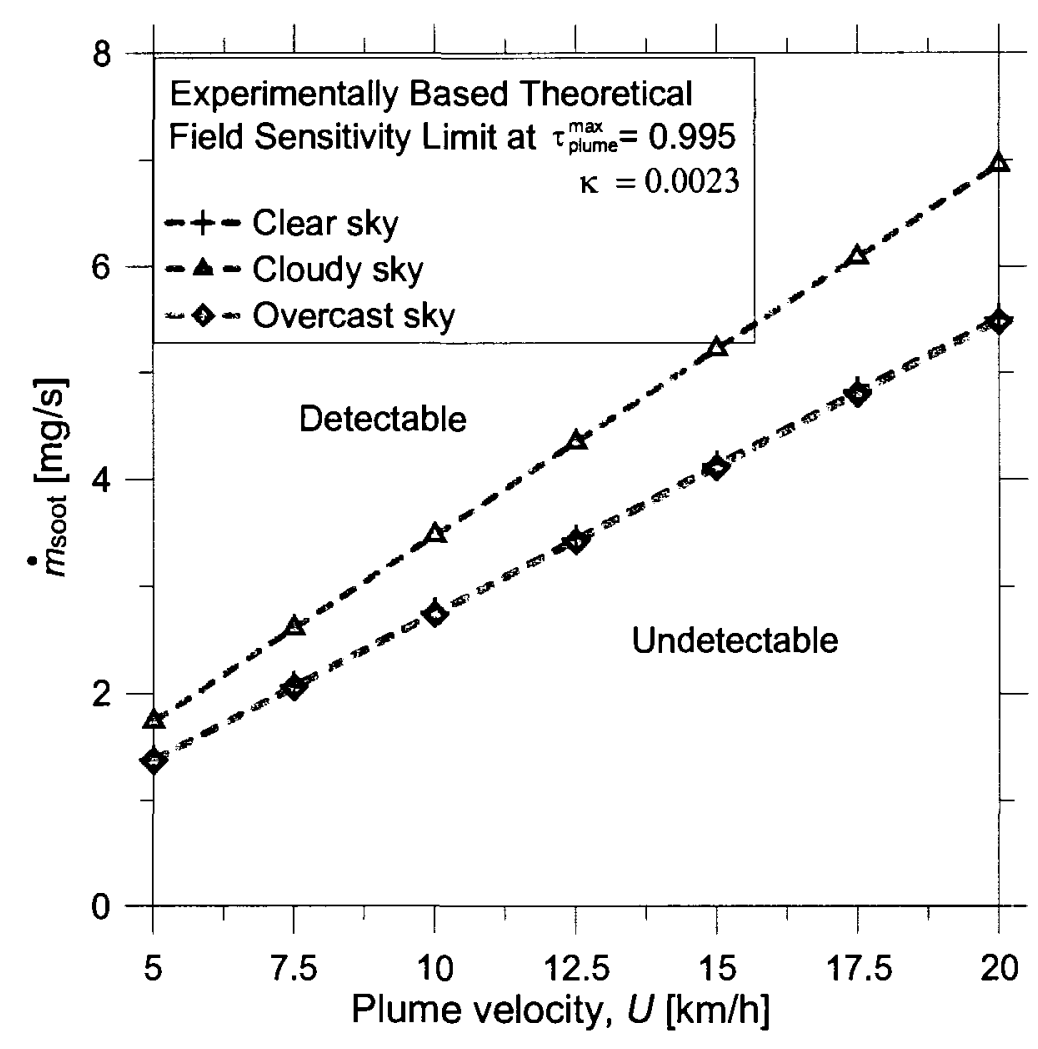

Figure 5.5: Detectable limits with sky-LOSA based on results of unconfined plume experiments assuming a 2 m diameter plume.

\subsection{Total Uncertainty: Error Propagation Analysis}

Error propagation analysis is used in cases where the quantity of interest is not measured directly but is calculated from measured quantities (each of which has a mean value and an uncertainty) through a functional relation. To estimate the achievable uncertainties in measuring soot mass flux, an error propagation analysis was performed on Equation 2.18 which is repeated below:

$$
\dot{m}_{\text {soot }}=\frac{u \rho_{\text {soot }} \lambda \cdot w}{6 \pi E(m)_{\lambda}\left(1+\rho_{\text {sa }}\right)} \kappa
$$


The quantity of $\dot{m}_{\text {soot }}$ that we wish to determine is not measured directly, but instead is functionally related with several measured variables such as $u, \rho_{\mathrm{soot}}, \lambda, E(m)_{\lambda}, \rho_{\mathrm{sa}, \lambda}$, and $\kappa$. The relative uncertainty in the soot emission rate is estimated as

$$
\frac{\sigma_{\dot{m}}}{\dot{m}}=\sqrt{\left(\frac{\sigma_{u}}{u}\right)^{2}+\left(\frac{\sigma_{\rho_{\mathrm{soot}}}}{\rho_{\mathrm{soot}}}\right)^{2}+\left(\frac{\sigma_{\lambda}}{\lambda}\right)^{2}+\left(\frac{\sigma_{E(m)_{\lambda}}}{E(m)_{\lambda}}\right)^{2}+\left(\frac{\sigma_{\rho_{\mathrm{sa}}}}{1+\rho_{\mathrm{sa}}}\right)^{2}+\left(\frac{\sigma_{\kappa}}{\kappa}\right)^{2}+\left(\frac{\sigma_{w}}{w}\right)^{2}}
$$

The basis of the quadrature addition is an assumption that the measured variables have a Gaussian distribution and the variables are independent of each other. The $\sigma$ terms are the standard deviation from the measurement of each variable and they are the contributions due to the uncertainties in each of the individual variables. The relative uncertainty contribution is calculated as if the given variable was the only source of uncertainty in the measurement. Overall Equation 5.1 determines how much $\dot{m}_{\text {soot }}$ would change if each variable were changed by its uncertainty. If we assume the uncertainty in $\lambda$ is sufficiently small not to contribute to the uncertainty in $\dot{m}_{\text {soot }}$, the Equation 5.1 becomes Equation 5.2 as follows

$$
\frac{\sigma_{\dot{m}}}{\dot{m}}=\sqrt{\left(\frac{\sigma_{u}}{u}\right)^{2}+\left(\frac{\sigma_{\rho_{\mathrm{soot}}}}{\rho_{\mathrm{soot}}}\right)^{2}+\left(\frac{\sigma_{E(m)_{\lambda}}}{E(m)_{\lambda}}\right)^{2}+\left(\frac{\sigma_{\rho_{\mathrm{sa}}}}{1+\rho_{\mathrm{sa}}}\right)^{2}+\left(\frac{\sigma_{\kappa}}{\kappa}\right)^{2}+\left(\frac{\sigma_{w}}{w}\right)^{2}}
$$

Equation 5.2 can be used to calculate the uncertainty of any individual sky-LOSA measurement. Here Table 5.1 summarizes the variables, uncertainties and relative contributions to the total error in $\dot{m}_{\text {soot }}$ from each of the variables based on lab-controlled plume measurements under different sky conditions. From Table 5.1, we can see the relative uncertainties from $\rho_{\text {soot }}, E(m)_{\lambda}, \rho_{\mathrm{sa}, \lambda}$, and $\kappa$ are of the same order, which 
means the uncertainty in $\dot{m}_{\text {soot }}$ could be improved by reducing the uncertainty in any one of the individual variables. The more significant sources of uncertainty are from $E(m)_{\lambda}$ and $\rho_{\mathrm{sa}, \lambda}$. For the present measurements, a constant $E(m)_{\lambda}$ function with a magnitude of 0.258 at a wavelength of $577 \mathrm{~nm}$ is assumed. In the future, $E(m)_{\lambda}$ and $\rho_{\text {sa }, \lambda}$ could be determined ex situ to the current experiment (refer to Coderre et al., 2008). For measurements on the lab scale plume with $\kappa=0.0022 \sim 0.0029$, the estimated overall uncertainty in $\dot{m}_{\text {soot }}$ is $31.7 \%, 32.0 \%$ and $32.7 \%$ for clear, cloudy, and overcast sky conditions respectively. However, the contribution of uncertainty from the plume transmissivity measurements (uncertainty based on the diagnostic itself) using sky-LOSA is only $10 \%$. 
Table 5.1: Uncertainty analysis on unconfined plume experiments in the lab

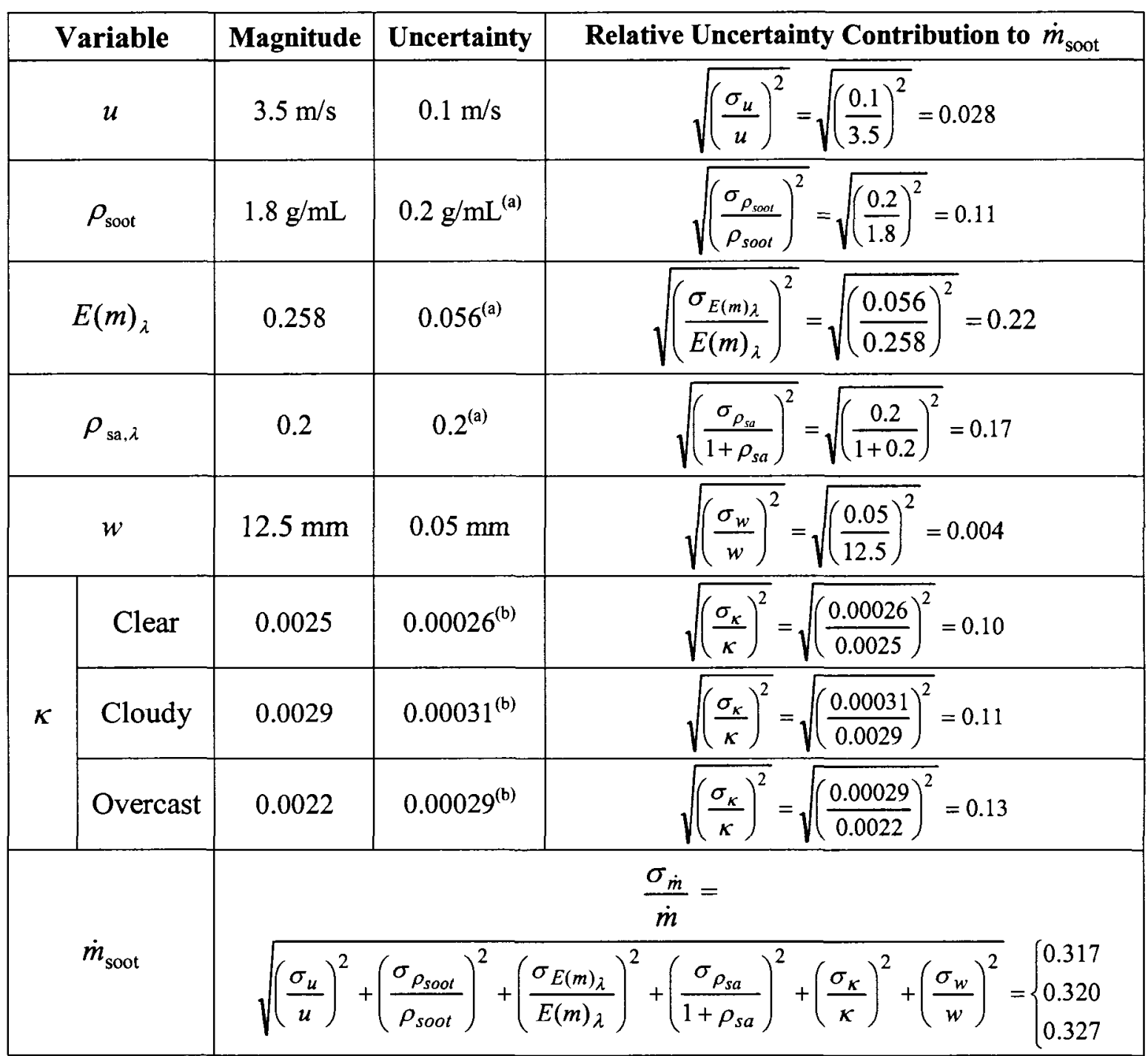

(a) The values of magnitude and uncertainty were adopted from the report [Johnson and Thomson, 2005]

(b) The values are obtained from the transmissivity measurements of plumes generated by the lab-controlled burner using sky-scattered radiation as the light source for test condition 6 .

\subsection{Applicability of Sky-LOSA to Measuring Soot from Solution Gas}

\section{Flares}

In this chapter, experiments were performed to estimate limiting uncertainties in using sky-scattered radiation to measure transmissivity of plumes generated by the labcontrolled burner in an outdoor environment. However, it is also desirable to estimate the 
achievable uncertainties with a sky-LOSA measurement of a full-size flare in the field. The lab-scale results show that, using the 3-image diffuse light technique as a standard, the highest plume transmissivity that could be measured in the field using skylight was $0.995(\kappa=0.0023)$. For a $2 \mathrm{~m}$-diameter plume moving with a $12.5 \mathrm{~km} / \mathrm{h}$ crosswind, the theoretical minimum mass flow rate of soot that could then be detected is $3.5 \mathrm{mg} / \mathrm{s}, 4.4$ $\mathrm{mg} / \mathrm{s}$, and $3.4 \mathrm{mg} / \mathrm{s}$ for clear, cloudy, and overcast sky conditions. Comparing these values with our target emission rate of $4.2 \mathrm{mg} / \mathrm{s}$, the current setup is on the threshold of being able to measure this target flow rate at this specific condition ( $2 \mathrm{~m}$-diameter plume at the velocity of $12.5 \mathrm{~km} / \mathrm{h}$ ) for various sky conditions.

To estimate achievable uncertainties in a field measurement, the error propagation analysis was repeated for an assumed set of field conditions for target soot yield rates of 4.2 and $42 \mathrm{mg} / \mathrm{s}$ as shown in Table 5.2. The uncertainty in $\kappa$ was the calculated mean of the uncertainties of $\kappa$ in lab-controlled plume measurements, which was shown to be essentially constant in Figure 5.4 above. This result shows that the uncertainty of the non-dimensional parameter $\kappa$ obtained from lab-scale plumes can be applied to practical-scale plumes in the field. 
Table 5.2: Uncertainty analysis for assumed field measurement conditions using sky-LOSA

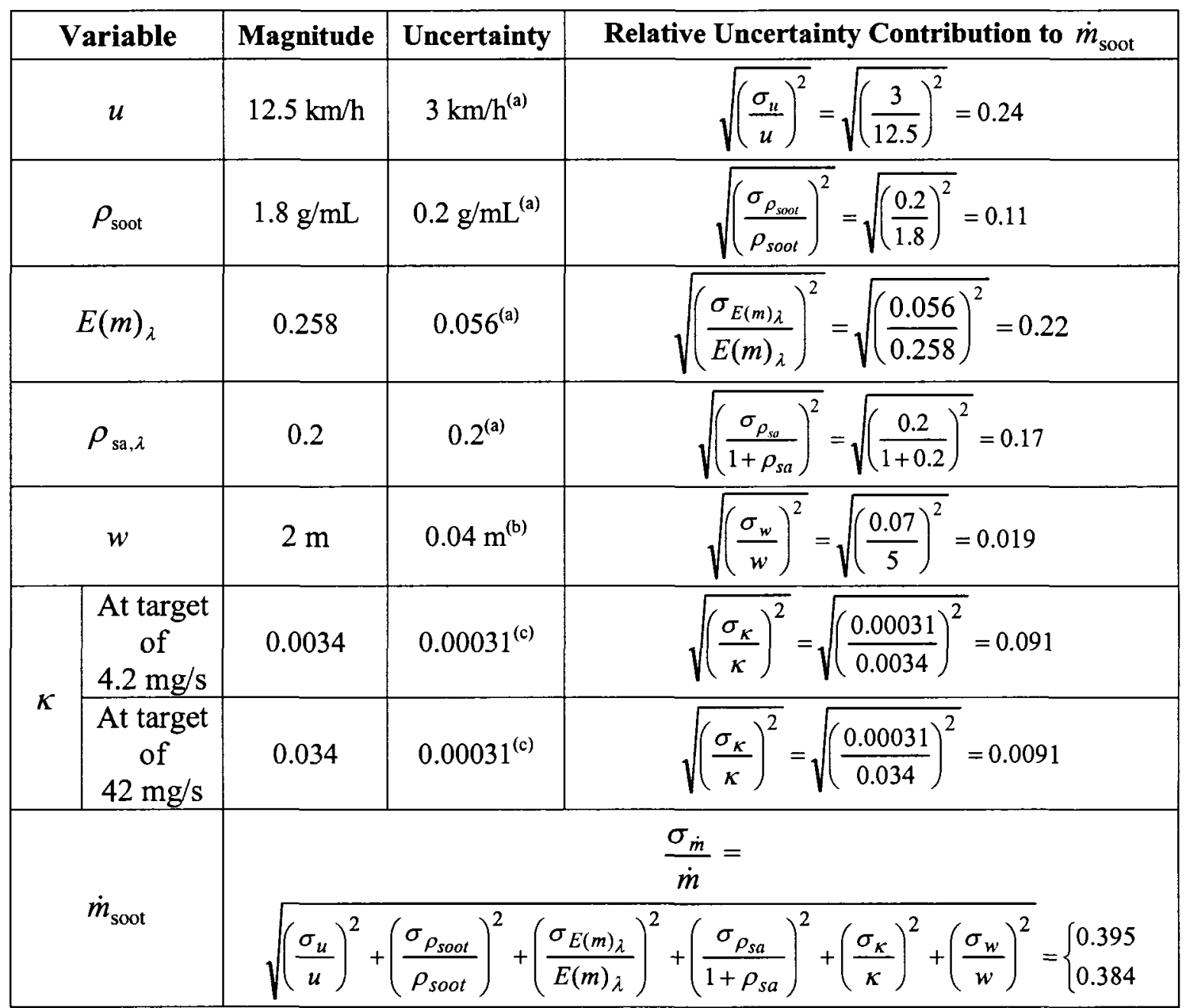

(a) The values of magnitude and uncertainty were adopted from the report [Johnson and Thomson, 2005]

(b) The relative uncertainty in plume width measurement was adopted from Section A.2.1 of Appendix.

(c) The values are obtained from the transmissivity measurements of plumes generated by the lab-controlled burner using sky-scattered radiation as the light source. The uncertainty in $\kappa$ was the calculated mean of uncertainties of $\kappa$ in lab-controlled plume measurements.

The results in Table 5.2 show that the more significant sources of uncertainty are from plume velocity and soot refractive index measurements. Further research into ways to accurately measure plume velocity (such as image correlation velocimetry or sonic detection and ranging (SODAR)) could also help reduce uncertainties. Compared to the contributions from $u$ and $E(m)_{\lambda}$, the uncertainty from $\kappa$ on which this project is 
focused, is less significant. Since the uncertainty in $\kappa$ is 'fixed', the relative uncertainty in $\kappa$ decreases one order (from $9.1 \%$ to $0.91 \%$ ) when the target soot emission rate increases one order (from 4.2 to $42 \mathrm{mg} / \mathrm{s}$ ). The estimated overall uncertainties in $\dot{m}_{\text {soot }}$ are $39.5 \%$ and $38.4 \%$ for target rates of 4.2 and $42 \mathrm{mg} / \mathrm{s}$, respectively where the contribution of uncertainty from the plume transmissivity measurements using sky-LOSA is $9.1 \%$ and $0.91 \%$, respectively. This result suggests that field measurements of soot plumes using sky-LOSA should be possible with overall uncertainties on the order of $40 \%$. Although this uncertainty is not insignificant, the sky-LOSA technique is potentially a major improvement over more qualitative opacity measurement techniques currently in use around the world. 


\section{Conclusions and Future Work}

\subsection{Conclusions}

This project is the first investigation of 2D-LOSA in an outdoor application for plume transmissivity measurements using sky-scattered solar radiation as the light source. This sensitivity and uncertainty limits of this new sky-LOSA technique were investigated experimentally. Applicability of sky-LOSA to a target application of measuring soot from solution gas flares was also considered.

In sky-LOSA, it is impossible to isolate the background from the attenuating medium in the field, so it is necessary to interpolate an appropriate background image in the region behind the plume. A series of experiments were performed to measure the uncertainties from the background interpolation for different plume sizes and under clear, cloudy, and overcast sky conditions. The background interpolation uncertainty depends strongly on the width of the plume relative to the image size, and weakly on the fit parameters when using Loess smoothing coupled with local polynomial interpolation. The experimental results show that background intensity in the plume region can be accurately interpolated from unattenuated intensity data outside of the region. Measurements appear possible under different sky conditions and the interpolation bias in $\kappa$ is around $2.32 \times 10^{-4}$ if the plume size in the image remains less than $20 \%$.

Examination of the effects of non-soot particles suggests that no water condensation will occur when the ambient temperature is above $-27^{\circ} \mathrm{C}$ and secondary particles 
(sulfates and nitrates) will not be present in the measurement location. Therefore, nonsoot particles should not interfere with the transmissivity measurements.

Experiments were also performed to determine the ultimate accuracy and sensitivity limit for transmissivity measurements using sky-scattered radiation for a lab-controlled plume under different sky conditions. The measurements show that a minimum transmissivity through the centre of the plume of 0.995 corresponding to $\kappa=0.0023$ could be detected and analyzed. The non-dimensional parameter $\kappa$ can be used to apply results from lab-scale plumes to practical-scale plumes in the field, due to its independence from local transmissivity variation across the plume and plume width. At this $\kappa$ limit, for a plume with a velocity of $12.5 \mathrm{~km} / \mathrm{h}$ (corresponding to a typical wind speed for flare locations in Alberta), the theoretical minimum detectable mass flow rates of soot in a $2 \mathrm{~m}$ wide plume are $3.5 \mathrm{mg} / \mathrm{s}, 4.4 \mathrm{mg} / \mathrm{s}$, and $3.4 \mathrm{mg} / \mathrm{s}$ for clear, cloudy, and overcast sky conditions, respectively. Comparing these values with our target emission rate of $4.2 \mathrm{mg} / \mathrm{s}$, the current setup is on the threshold of being able to measure this target flow rate at this specific condition ( 2 m-diameter plume at the velocity of $12.5 \mathrm{~km} / \mathrm{h}$ ) for various sky conditions.

An error propagation analysis on results of lab-controlled plume measurements showed that the overall uncertainty in $\dot{m}_{\text {soot }}$ was estimated to be $30 \%$ under different sky conditions, where an uncertainty of $10 \%$ attributable to the sky-LOSA measurements of $\kappa$. For a range of assumed field conditions, a further uncertainty calculation showed that overall uncertainties in $\dot{m}_{\text {soot }}$ of approximately $40 \%$ are achievable in the field where the most significant uncertainties arise from the estimation of plume velocity and soot optical 
properties. The uncertainty from the sky-LOSA measurements decreases with increasing the value of target rates.

From this investigation, it can be concluded that sky-scattered solar radiation can be effectively used as a light source for plume transmissivity measurements in the field. The experimental and analytical results presented herein suggest that the sky-LOSA technique is likely to be a significant improvement over the existing qualitative methods based on opacity.

\subsection{Future Work}

The results of this thesis have shown sky-LOSA to be a promising technique for field measurement of soot flux in plumes. However, further research is recommended to improve the understanding of uncertainties caused by other parameters related to the quantitative measure of soot emission rates.

\subsubsection{Direct Sunlight Scattering Effect}

In sky-LOSA, the sky scattered solar radiation is used as the source of light. A potentially significant interference is the effect of sunlight scattered directly by the plume. Since theoretical studies of this problem are very rare in the literature, it becomes necessary to study this effect experimentally. In the present experiment, direct sunlight was blocked by a black screen to avoid direct scattering of sunlight (see Figure $3.8 \mathrm{~b}$ ). The order of magnitude of this direct solar scattering effect could be estimated by taking sky-LOSA measurements with and without the screen on a clear sunny day. However, a lens with an appropriate neutral density filter would be required to avoid saturating the $\mathrm{CCD}$. To minimize direct solar radiation scatter bias in an in-field application, it would 
be beneficial to make spectral measurements to aid in the selection of an optimal wavelength that is more sensitive to skylight and less sensitive to sunlight.

\subsubsection{Wind Speed Measurement}

Wind speed is another parameter that needs to be evaluated for in-field applications. The wind velocity field can be measured using anemometers mounted at the emission site. However, in general it is not possible to mount anemometers at the stack height of flares and plume velocity must be estimated by other means. This can cause large uncertainties in the deduced wind speed and, consequently, large uncertainties in the estimated flare flow rate.

Another method of measuring wind speed is to use SODAR (sound detection and ranging), which uses sound waves and the Doppler principle to measure the wind profile. The Doppler SODAR sends out sound pulses of several frequencies in slightly different directions. The acoustic signals are backscattered by inhomogeneities in the atmosphere. The velocity of the plume is then deduced from the frequency shifts of the return signals [Schroter et al., 2003]. The sodar technique often requires bulky extra equipment.

Weibring et al. (1998) proposed a simple and inexpensive wind speed measurement method employing an ordinary CCD camera, which could thus be easily incorporated into an existing optical diagnostic system. This second camera takes pictures of the most visible part of the plume, allowing the wind speed to be estimated by measuring the displacement of the plume structure between subsequent images. In principle, this technique might be applied to the raw transmissivity images in a turbulent plume. 


\section{References}

Particle Instruments. (2006). Aerosol Instrument Scanning Mobility Particle Sizer Manual. TSI Incorporated.

Alastuey, A., Querol, X., Rodriguez, S., Plana, F., Lapez-soler, A., Ruiz, C., \& Mantilla, E. (2004). Monitoring of atmospheric particulate matter around sources of secondary inorganic aerosol. Atmospheric Environment. Vol. 38, 4979-4992.

Anlauf, K.G., Fellin, P., \& Wiebe, H.A. (1982). The Naniticoke shoreline diffusion experiment, June 1978-IV. A. Oxidation of sulphur dioxide in a power plant plume. B. Ambient concentrations and transport of sulphur dioxide, particulate sulphate and nitrate, and ozone. Atmospheric Environment. Vol. 16, 455-466.

API (1969). Guide for pressure-relieving and depressuring systems - American Petroleum Institute Recommended Practice 521'. Washington, D.C.: American Petroleum Institute, Edition 1, 1969.

Bockhorn, H. (Ed.). (1994). Soot Formation in Combustion Mechanisms and Models. Berlin Heidelberg: Springer-Verlag.

Bogumil, K., Orphal, J., Homann, T., Voigt, S., Spietz, P., Fleischmann, O.C., Vogel, A., Hartmann, M., Kromminga, H., Bovensmann, H., Frerick, J., \& Burrow, J.P. (2003). Measurements of molecular absorption spectra with the SCIAMACHY pre-flight model: instrument chareacterization and reference data for atmospheric remote-sensing in the $230-2380 \mathrm{~nm}$ region. Journal of Photochemistry and Photobiology A: Chemistry, Vol. 157 (2-3), 167-184.

Bohren, C.F., \& Huffman, D. (1983). Absorption and scattering of light by small particles. New York: John Wiley \& Sons, Inc.

Brzustowski, T. A., Gollahalli, S. R., Gupta, M. P., Kaptein, M. \& Sullivan, H. F. (1975). Radiant heating from flares', ASME paper 75-HT-4, Heat Transfer Conference, August 11-13, 1975. San Francisco, California. 
Canteenwalla, P.M. (2007). Soot emissions from turbulent diffusion flames burning simple Alkane fuels. Master thesis. Ottawa, Ontario, Canada.

Canadian Council of Ministers of the Environment (CCME) (2005). Emission monitoring and reporting strategy -summary and background. Retrieved April, 15, 2008, from

http://www.ccme.ca/assets/pdf/e_m strategy_summary_final_eng.pdf

Cheng, L., Peake, E., \& Davis, A. (1987). The rate of $\mathrm{SO}_{2}$ to sulphate particle formation in an air parcel from an oil sands extraction plant plume. Journal of the Air Pollution Control Association. Vol. 37, 163-167.

Cheng, L., Sandhu, H.S., Angle, R.P., McDonald, K.M., \& Myrick, R.H. (2000). Rural particulate matter in Alberta, Canada. Atmospheric Environment. Vol. 34, 33653372.

Chippett, S. \& Gray, W. A. (1978). The size and optical properties of soot particles. Combustion and Flame, Vol. 31, 149-159.

Cleveland, W.S. and Loader, C. (1996). Smoothing by Local Regression: Principles and Methods. In W. Haerdle and M.G. Schimek, editors, Statistical Theory and Computational Aspects of Smoothing. New York: Springer, 10-49.

Coderre, A.R., Snelling, D.R., Smallwood, G.J., \& Johnson, M.R. (2007). Measuring optical properties of cooled post-flame soot. Proceedings from CI/CS 2007: The 2007 Spring Technical Meeting of the Combustion Institute Canadian Section. Banff, Alberta, Canada.

Coderre, A.R., Snelling, D.R., Smallwood, G.J., \& Johnson, M.R. (2008). Measuring optical properties of cooled soot. Proceedings from CI/CS 2008: The 2008 Spring Technical Meeting of the Combustion Institute Canadian Section. Toronto, Ontario, Canada.

Collis, R.T.G. \& Uthe, E.E. (1972). Mie scattering techniques for air pollution measurement with lasers. Opto-electronics, Vol. 4, 87-99.

Colls, J. (2002). Air Pollution (2 ed.). London and New York: Spon Press.

Cook, C.S., Bethke, G.W., \& Conner, W.D. (1972). Remote measurement of smoke plume transmittance using lidar. Applied Optics, Vol.11, No. 8, 1742-1748. 
Cook, D.K., Fairweather, M., Hammonds, J. \& Hughes, D.J. (1987). Size and radiative characteristics of natural gas flares. Part 1- field scale experiments. Chemical Engineering Research and Design. Vol. 65, 318-325.

Daun, K.J., Thomson, K.A., Liu, F. and Smallwood, G.J. (2006). Deconvolution of axisymmetric flame properties using Tikhonov regularization. Applied Optics, Vol.45, 4638-4646.

Dalzell, W. H. \& Sarofim, A. F. (1969). Optical constants of soot and their application to heat flux calculations. Transactions of the ASME. Journal of Heat Transfer, 91, 100.

Dasch, C.J. (1992). One-dimensional tomography: a comparison of Abel, onion-peeling, and filtered backprojection methods. Applied Optics, Vol. 31, 1146-1152.

De Nevers, N. (2000). Air pollution control engineering ( $2^{\text {nd }}$ ed.). McGraw-Hill.

Devore, J.L. (2004). Probability and statistics for engineering and the sciences ( $6^{\text {th }}$ ed.). USA: Brooks/Cole-Thomson Learning.

Dobbins, R.A. \& Megaridis, C.M. (1991). Absorption and scattering of light by polydisperse aggregates. Applied Optics, Vol. 30, No. 33, 4747-4754.

Dobbins, R.A., Mulholland, G.W., Bryner, N.P. (1994). Comparison of a fractal smoke optics model with light extinction measurements. Atmospheric Environment, Vol. 28 No. $5,889-897$.

Faeth, G.M., \& Koylu U.O. (1995). Soot morphology and optical properties in nonpremixed turbulent flame environments. Combustion Science and Technology, Vol.108, No.4-6, 207-229.

Farias, T.L., Koylu, U.O., \& Garvalbo, M.G. (1996). Range of validity of the RayleighDebye-Gans theory for optics of fractal aggregate. Applied Optics, Vol. 35, No. 33, 6560-6567.

Flower, W.L., \& Bowman, C.T. (1986). Soot production in axisymmetric laminar diffusion flames at pressures form one to tem atmospheres. Proc. Combust. Inst., Vol. 21, 1115-1124.

Forrest, J., Garber, R., \& Newman, L. (1981). Conversion rates in power plant plumes based on filter pack data: the coal-fires Cumberland plume. Atmospheric Environment. Vol. 13, 1287-1297. 
Frenklach, M. (2002). Reaction mechanism of soot formation in flames. Physical Chemistry Chemical Physics, Vol. 4, 2028-2037.

Gillani, N.v. \& Wilson, W.E. (1980). Formation and transport of ozone and aerosols in power plant plumes. Annals of the New York Academy of Sciences, Vol. 338, 276296.

Gillani, N.V. \& Kohli, S. (1981). Gas-to-particle conversion of sulfur in power plant plumes - I. parametrization of the conversion rate for dry, moderately polluted ambient conditions. Atmospheric Environment. Vol. 15, No. 10/11, 2293-2313.

Gillani, N.V., Luria, M., Valente, R.J., Tanner, R.L., Imhoff, R.E., \& Meagher, J.F. (1998). Loss rate of Noy from a power plant plume based on aircraft measurements. Journal of Geophysical Research. Vol. 103, 22585-22592.

Glassman, I. (1988). Soot formation in combustion processes. 22 $2^{\text {nd }}$ Symposium (International) on Combustion, The combustion Institute, 295-311.

Glassman, I. (1996). Combustion ( ${ }^{\text {rd }}$ ed.). San Diego, California: Academic Press.

Gore, J.P. and Faeth, G.M. (1986). Structure and radiation properties of turbulent ethylene/air diffusion flames. Proceeding of the Twenty-First Symposium (International) on Combustion, The Combustion Institute, Pittsburgh, PA., 15211531.

Greenberg, P. S. \& Ku, J.C. (1997). Soot volume fraction imaging. Applied Optics, Vol. 36, No. 22, 5514-5522.

Guigard S.E. \& Kindzierski, W. B. (2000). Heat radiation from flares. A report to Alberta Environment.

Gulder, O.L. (1999). Soot particulate formation and characterization in combustion. Transaction of the CSME, Vol. 23, No. 1B, 225-240.

Hausdorff, F. (1918). Dimension und äußeres Maß. Mathematische Annalen. Vol. 79, No. 1-2, 157-179.

Hecht, E. (2006). Optics ( $2^{\text {nd }}$ ed.). South Asia: Pearson Education.

Hewitt, C.N. (2001). The atmospheric chemistry of sulphur and nitrogen in power station plumes. Atmospheric Environment. Vol. 35, 1155-1170.

Intergovernmental Panel on Climate Change (IPCC) (2007). Working Group 1: The physical science basis of climate change. AR4 report. 
Johnson, M.R., Spangelo, J.L. \& Kostiuk, L.W. (2001). A characterization of solution gas flaring in Alberta. Journal of the Air and Waste Management Association, Vol. $51,1167-1177$.

Johnson, M.R. \& Thomson, K.A. (2005). Direct solar radiation based plume opacity measurements. Final Report to Environment Canada.

Julien, R. \& Botet, R. (1987). Aggregation and Fractal Aggregates. Singapore: World Scientific Publishing Co.

Ku, J.C. \& Shim, K.H. (1992). A comparison of solutions for light scattering and absorption by agglomerated or arbitrarily-shaped particles. Journal of Quantitative Spectroscopy and Radiative Transfer, Vol. 47, Issue 3, 201-220.

Koylu, U.O. \& Faeth, G.M. (1994a). Optical properties of overfire soot in buoyant turbulent diffusion flames at long residence times. Journal of Heat Transfer, Vol. $116,152-159$.

Koylu, U.O. \& Faeth, G.M. (1994b). Optical properties of soot in buoyant laminar diffusion flames. Journal of Heat Transfer, Vol. 116, 971-979.

Koylu, U.O. \& Faeth, G.M. (1996). Spectral extinction coefficients of soot aggregates from turbulent diffusion flames. Journal of Heat Transfer Vol. 118, 415-421.

Koylu, U.O., Xing, Y., \& Rosner, D.E. (1995). Fractal morphology analysis of combustion-Generated aggregates using angular light scattering ad electron microscope images. Langmuir, Vol. 11, 4848-4854.

Krishnan, S.S., Lin, K.C., \& Faeth, G.M. (2000). Optical properties in the visible of overfire soot in large buoyant turbulent diffusion flames. Journal of Heat Transfer. Vo. 122, 517-524.

Krishnan, S.S., Lin, K.C., \& Faeth, G.M. (2001). Extinction and scattering properties of soot emitted from buoyant turbulent diffusion flames. Journal of Heat Transfer. Vo. 123, 331-339.

Lazaridis, M., Isukapalli, S.S., \& Georgopoulos, P.G. (2001). Modelling of aerosol processes in plume. Tellus. 53B. 83-93.

Lee, S. C. \& Tien, C. L. (1980). Optical constants of soot in hydrocarbon flames. Eighteenth Symposium (International) on Combustion, The Combustion Institute, Pittsburgh, 1159-1166. 
Liebsch, E.J. \& De Pena, R.G. (1982). Sulphate aerosol production in coal-fired power plant plumes. Atmospheric Environment. Vol. 16, 1323-1331.

Lilienfeld, P., Woker, G, Stern, R. \& McVay, L. (1981). Passive remote smoke plume opacity sensing. Applied Optics, Vol. 20, No. 5, 800-806.

Lou, W. \& Charalampopoulos, T.T. (1994). On the electromagnetic scattering ad absorption of agglomerated small spherical particles. Journal of Physics D: Applied Physics, Vol. 27, 2258-2270.

Lusis, M.A., Anlauf, K.G., Barrie, L.A. \& Wiebe, H.A. (1978). Plume chemistry studies at a northern Alberta power plant. Atmospheric Environment. Vol. 12, 2429-2437.

Mamane, Y. \& Pueschel, R.F. (1980). Formation of sulphate particles in the plume of the Four Corners power plant. Journal of Applied Meteorology. Vol. 19, 779-790.

Mathcad Help version 13, Mathematical software for PCs from Parametric Technology Corporation (PTC), Needham, MA, USA.

Meagher, J.F., Stockburger, L., Bailey, E.M., \& Huff, O. (1981). Atmospheric oxidation of flue gases from coal fired power plants - a comparison between conventional and scrubbed plumes. Atmospheric Environment. Vol. 15, 749-762.

McFarland, M.J. \& Terry, S.H. (2003). Evaluation of the digital opacity compliance system in high mountain desert environments. Journal of the Air and Waste Management Association, Vol. 53, 724-730.

McFarland, M.J. \& Terry, S.H. (2004). Measuring visual opacity using digital imaging technology. Journal of the Air and Waste Management Association, Vol. 54, 296306.

McFarland, M.J., Rasmussen, S.L., Stone, D.A., Palmer, G.R. \& Wander, J.D. (2006). Validation of the digital opacity compliance system under regulatory enforcement conditions. Journal of the Air and Waste Management Association, Vol. 56, 12601266.

Morrison, I.D. \& Ross, S. (2002). Colloidal Dispersions: Suspensions, Emulsions and Foams. New York: John Wiley \& Sons, Inc.

Mueller, S.F. \& Imhoff, R.E. (1994a). Estimates of particle formation and growth in coalfired boiler exhaust - I. observation. Atmospheric Environment. Vol. 28, No. 4, $595-602$. 
Mueller, S. F. \& Imhoff, R.E. (1994b). Estimates of particle formation and growth in coal-fired boiler exhaust - II. Theory and model simulations. Vol. 28, No. 4, 603610.

Musculus, M.P.B. \& Pickett, L.M. (2005). Diagnostic considerations for optical laserextinction measurements of soot in high-pressure transient combustion environments. Combustion and Flame, Vol. 141, 371-391.

Peters, A., Dockery, D.W., Muller, J.E., \& Mittleman, M.A. (2001). Increased particulate air pollution and the triggering of myocardial infarction. Circulation, 103, 28102815.

Pope, C.A., Burnett, R.T., Thun, M.J., Calle, E.E., Krewski, D. Ito, K., \& Thurston, G.D. (2002). Lung Cancer, cardiopulmonary mortality, and long-term exposure to fine particulate air pollution. Journal of the American Medical Association, 287 (9), 1132-1141.

Poudenx, P. (2000). Plume sampling of a flare in crosswind: structure and combustion efficiency. Ph. $D$ thesis. Edmonton, Alberta, Canada.

Province of Alberta, ALBERTA REGULATION 124/93. (2005). Environmental Protection and Enhancement Act. SUBSTANCE RELEASE REGULATION, AR 124/93 s4; 191/96; 159.

Querol, X., Alastuey, A., Lopez-soler, A., Mantilla, E., and Plana, F. (1996). Mineral composition of atmospheric particulates around a large coal-fired power station. Atmospheric Environment. Vol. 30, No. 21, 3557-3572.

Querol, X., Alastuey, A. Lopez-soler, A., Plana, F., Mantilla, E., Juan, R., Ruiz, C.R. \& Orden, A.L. (1999). Characterisation of atmospheric particulates around a coalfried power station. International Journal of Coal Geology. Vol. 40, 175-188.

Quinten, M., Friehmelt, R. \& Ebert, K.F. (2001). Sizing of aggregates of spheres by a white-light optical particle counter with 900 scattering angle. Journal of Aerosol Science, Vol. 32 issue 1, 32-72.

Ramanathan, V. \& Carmichael, G. (2008). Global and regional climate changes due to black carbon. Nature Geoscience, Vol. 1, 221-227.

Rossman, R.P., \& Smith, W.R. (1943). Density of carbon black by helium displacement. Industrial \& Engineering Chemistry, Vol. 35, Iss.9, 972-976. 
Siegel, R. \& Howell, J.R. (1992). Thermal Radiation Heat Transfer $\left(3^{\text {rd }}\right.$ ed.). Washington DC: Taylor \& Francis.

Schroter, M., Obermeier, A., Bruggemann, D., Plechschmidt, M., \& Klemm, O. (2003). Remote monitoring of air pollutant emissions from point sources by a mobile Lidar/Sodar system. Journal of the Air \& Waste Management Association, Vol. $53,716-723$.

Schwartz, J., Norris, G., Larson, T., Sheppard, L., Claiborne, C., \& Koenig, J. (1999). Episodes of high coarse particle concentrations are not associated with increased mortality. Environmental Health Perspectives, Vol. 107, 339-342.

Shi, Z., Shao, L., Jones, T.P., Whittaker, A.G., Lu, S., Berube, K.A., He, T., \& Richards, R.J. (2003). Characterization of airborne individual particles collected in an urban area, a satellite city and a clean air area in Beijing, 2001. Atmospheric Environment AE International-Asia. Vol. 37, 4097-4108.

Siegell, R., \& Howell, J. R. (1981). Thermal Radiation and Heat Transfer, $2^{\text {nd }}$ ed. McGraw-Hill, New Youk, 450-595.

Smyth, K. C., \& Shaddix, C. R. (1996). The elusive history of $m=1.57-0.56 \mathrm{i}$ for the refractive index of soot. Combustion and Flame, 107, 314-320.

Snelling, D. R, Thomson, K. A., Smallwood, G. J., \& Gulder, O. L. (1999). Twodimensional imaging of soot volume fraction in laminar diffusion flames. Applied Optics, Vol. 38, No. 12, 2478-2485.

Snelling, D.R. (2007 \& 2008) Personal communications.

Sonntag, R.E. \& Van Wylen, G.J. (1985). Fundamentals of Classical Thermodynamics $\left(3^{\text {rd }}\right.$ ed.). New York: John Wiley \& Sons.

Sorensen, C.M., Cai, J., \& Lu, N. (1992). Light-scattering measurements of monomer size, monomers per aggregate and fractal dimension for soot aggregates in flames. Applied Optics, Vol. 31, 6547-6557.

Stipe, C.B., Higgins, B.S., Lucas, D., Koshland, C. P., \& Sawyer, R.F. (2005). Inverted co-flow diffusion flame for producing soot. Review of Scientific Instrument, 76, $023908,1-5$.

Tan, S. H. (1967). Flare system design simplified. Hydrocarbon Processing, Vol. 46, No. 1, January 1967, 72-176. 
Tang, I.N. (1996). Chemical and size effects of hygroscopic aerosols on light scattering coefficients. Journal of Geophysical Research. Vol. 101, No. D14, 19245-19250.

Thomson, K.A. (2000). Soot Temperature Measurements by Spectral Emission Methods in Laminar Diffusion Flame. Master thesis.(University of Waterloo, Ontario, Canada, 2000)

Thomson, K. A. (2004). Soot Formation in Annular Non-premixed laminar flames of methane-air at pressures of 0.1 to 4.0 MPa. Ph.D. dissertation (University of Waterloo, Ontario, Canada, 2004).

Thomson, K.A., Gülder, O.L., Weckman, E.J., Fraser, R.A, Smallwood, G.J., Snelling, D.R. (2005). Soot concentration and temperature measurements in annular, nonpremixed laminar flames at pressures up to $4 \mathrm{MPa}$. Combustion and Flame, Vol. $140,222-232$.

Thomson, K.A., Johnson M.J., Snelling, D.R., \& Smallwood, G.J. (2008). Diffuse-light two-dimensional line-of-sight attenuation for soot concentration measurements. Applied Optics Vol. 47 No.5, 694-703.

Thomson, K. A., Johnson, M.R., Snelling, D.R. \& Smallwood, G.J. (2008b). Onedimensional spectral line-of-sight attenuation for soot optical property measurement. Proceedings from CI/CS 2008: The 2008 Spring Technical Meeting of the Combustion Institute Canadian Section. Toronto, Ontario, Canada.

Tian, K., Thomson, K.A., Liu, F., Snelling, D.R., Smallwood, G.J., \& Gulder, O.L. (2004). Determination of the soot absorption function and thermal accommodation coefficient using low-fluence LII in a laminar coflow ethylene diffusion flame. Combustion and Flame, Vol. 136, 180-190.

Tsang, L., Kong, J.A., \& Ding, K. (2000). Scattering of Electromagnetic Waves. New York: a Wiley-Interscience Publication.

Turns, S.R. (1996). An Introduction to Combustion - Concepts and Applications ( $1^{\text {st }}$ ed.). New York: McGraw-Hill Inc.

US EPA (1971). Method 9 - Visual determination of the opacity of emissions from stationary sources. Electronic Code of Federal Regulations 40 - Protection of the Environment Part 60 - Standards of performance for new stationary source Appendix A-4, http:// www.epa.gov/ttn/emc/promgate/m-09.pdf. 
US EPA (1975). Evaluation and collaborative study of method for visual determination of opacity of emissions from stationary sources. Contract No. 68-02-0626.

US EPA (1997). National ambient air quality standards for particulate matter; Final Rule. Federal Register, 40 CFR Part 50, [AD-FRL-5725-2]. RIN 2060-AE66.

U.S. EPA (2004). Air quality criteria for particulate matter. United States Environmental Protection Agency (U. S. EPA), Vol. I and II, 2048 Pages.

VanderWal, R.L., Jensen, K.A., \& Choi, M.Y. (1997). Simultaneous laser-induced emission of soot and polycyclic aromatic hydrocarbons within a gas-jet diffusion flame. Combustion and Flame, Vol. 109 (3): 399-414.

Wang, G. \& Sorensen, C.M. (2002). Experimental test of the Rayleigh-Debye-Gans theory for light scattering by fractal aggregates. Applied Optics, Vol. 41, No. 22, 4645-4651.

Weinberg, F.J. (1963). Optics of Flames: including methods for the study of refractive index fields in combustion and aerodynamics. London: Butterworths.

Weibring, P., Andersson, M. Edner, H. \& Svanberg, S. (1998). Remote monitoring of industrial emissions by combination of lidar and plume velocity measurements. Applied Physics B-Lasers and Optics, Vol. 66, 383-388.

Wu, J.S., Krishnan, S.S., \& Faeth, G.M. (1997). Refractive indices at visible wavelengths of soot emitted from buoyant turbulent diffusion flames. Journal of Heat Transfer. Vol. 119, 230-237.

Zabetakis, M. G. \& Burgess, D. S. (1961). Research on the hazards associated with the production and handling of liquid hydrogen, R.I. 5707, U.S. Bureau of Mines, 1961.

Zak, B.D. (1981). Lagrangian measurements of sulphur dioxide to sulphate conversion rates. Atmospheric Environment. Vol. 15, 2583-2591.

Zhao, Y., Brewer, W.A., Eberhard, W.L. \& Alvarez, R. J. (2002). Lidar measurement of Ammonia concentrations and fluxes in a plume from a point source. Journal of Atmospheric and Oceanic Technology, Vol. 19, 1928-1938. 


\section{APPENDIX A: Spatial Calibration for Field Application}

\section{A.1 Introduction}

From previous chapter we know that the soot mass emission rate is calculated by integrating the logarithm of the plume transmissivity across the width of the plume (see Equation A.1). In the diagnostic we are imaging the plume and therefore we must express Equation A.1 in terms of the image dimensions, i.e., pixels. Note the relationship between y and pixel as Equation A.2.

$$
\begin{gathered}
\dot{m}_{\mathrm{soot}}=\frac{u \rho_{\mathrm{soot}} \lambda}{6 \pi E(m)_{\lambda}\left(1+\rho_{\mathrm{sa}, \lambda}\right)} \int \ln \left(\tau_{\lambda}\right) \mathrm{d} y \\
y=\frac{\text { pixel } \times \text { pixelsize }}{M}
\end{gathered}
$$

where pixel size is the width of a CCD pixel as specified by the CCD manufacturer and $M$ is the magnification of the optics (defined as in Equation A.5). Combining Equations A.1 and A.2, we create an expression for soot mass emission rate in terms of the image pixel dimension:

$$
\dot{m}_{\text {soot }}=\frac{u \rho_{\text {soot }} \lambda}{6 \pi E(m)_{\lambda}\left(1+\rho_{\mathrm{sa}}\right)} \cdot \frac{\text { Pixelsize }}{M} \int \ln \left(\tau_{\lambda}\right) \mathrm{d} \text { pixel }
$$

Magnification calibration refers to the process of correlating the pixels of imaged objects to their actual size. In a field magnification calibration, we wish to calculate $M$ of an image as well as the uncertainty of $M$. If assumed the uncertainty of magnification is 
the only source of uncertainty in the measurement, the relative uncertainty in soot mass yield rate $\left(\sigma_{\dot{m}_{\text {soot }}} / \dot{m}_{\text {soot }}\right)$ can be obtained:

$$
\frac{\sigma_{\dot{m}_{\text {soot }}}}{\dot{m}_{\text {soot }}}=\sqrt{\left(\frac{\sigma_{\mathrm{M}}}{M}\right)^{2}}
$$

Therefore, the relative uncertainty of $\dot{m}_{\text {soot }}$ scales with the relative uncertainty of $M$.

\section{A.2 Field Magnification Calibration}

Calculation of $M$ in a lab setting is straightforward. After taking an image of a target with known dimension and measuring the target image size in pixels, we obtain a magnification scale to convert size in pixels to actual size (e.g., millimeter). In the field setting, however, it is not practical to place a target of known size in a plume. Therefore, it is necessary to develop an indirect method for field spatial calibration and to estimate the uncertainty of the method.

In this section we outline a procedure to calculate $M$ and $\sigma_{\mathrm{M}}$ in a field setting based on knowledge of the lens focal length and the distance from the lens to the plume as measured using a laser range finder. Since the plumes in the field will typically be 10 to 100 meters away, it reasonable to assume the camera is perpendicular to and far away from a scene, so perspective and lens distortion are negligible.

Before discussing the procedure of calibration, it is useful to briefly introduce some fundamental relationships in optics which are used in the calibration (see Figure A.1). 


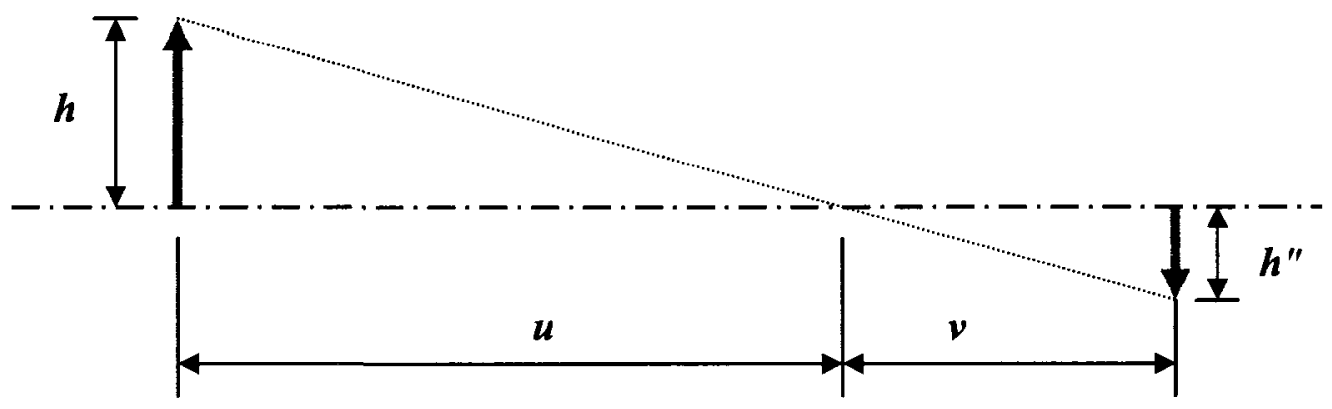

Figure A.1: Notation of terms.

As in Equation A.5, magnification is the ratio of image size to object size. It is also the ratio of distances from object to lens and image to lens.

$$
M=\frac{v}{u}=\frac{h^{\prime \prime}}{h},
$$

where $u$ and $v$ are named object distance and image distance, respectively; $h$ and $h^{\prime \prime}$ are called object height and image height, respectively. The relationship between focal length, object distance, and image distance is given by

$$
\frac{1}{f}=\frac{1}{u}+\frac{1}{v},
$$

where $f$ is the effective focal length. Combining Equations A.5 and A.6, we obtain:

$$
f=\frac{M \cdot u}{M+1},
$$

or

$$
M=\frac{f}{u-f}
$$

Equations A.7 and A.8 are just different versions of the same formula; the form used to solve a particular parameter will depend on which variables are known, and which are to be solved for. 
The uncertainty of magnification, $\sigma_{\mathrm{M}}$, can be calculated by performing an error propagation analysis on Equation A.8.

$$
\left(\frac{\sigma_{\mathrm{M}}}{M}\right)^{2}=\left(\frac{\frac{\partial}{\partial u}(M)}{M} \cdot \sigma_{\mathrm{u}}\right)^{2}+\left(\frac{\frac{\partial}{\partial f}(M)}{M} \cdot \sigma_{\mathrm{f}}\right)^{2}=\left(\frac{\sigma_{\mathrm{u}}}{f-u}\right)^{2}+\left(\frac{\sigma_{\mathrm{f}} \cdot u}{f(u-f)}\right)^{2}
$$

where $\sigma_{\mathrm{u}}$ is the absolute uncertainty of object distance and $\sigma_{\mathrm{f}}$ is the absolute uncertainty of focal length. In a field setting, the distance from lens to object is determined using a range finder and the uncertainty of the measurement is specified by the range finder manufacturer as 15 centimeters.

Conversely, the focal length of the lens is known only nominally to be $105 \mathrm{~mm}$ with an unknown uncertainty. The focal length of the lens can be calculated via Equation A.7 and the uncertainty of $f$ can be calculated following an error propagation analysis on Equation A.7.

$$
\left(\frac{\sigma_{\mathrm{f}}}{f}\right)^{2}=\left(\frac{\frac{\partial}{\partial u}(f)}{f} \cdot \sigma_{\mathrm{u}}\right)^{2}+\left(\frac{\frac{\partial}{\partial M}(f)}{f} \cdot \sigma_{\mathrm{M}}\right)^{2}=\left(\frac{\sigma_{\mathrm{u}}}{u}\right)^{2}+\left(\frac{\sigma_{\mathrm{M}}}{M(M+1)}\right)^{2}
$$

The measurement of the focal length of the lens is achieved using a Nikon D50 digital camera (pixel size $=7.8 \mu \mathrm{m} \times 7.8 \mu \mathrm{m}$ ) with a Micro-Nikkor $105 \mathrm{~mm}$ lens. The calibration procedure with a sample calculation is described below.

1. Position the target along the optical axis of the Nikkor lens. Set the camera in manual mode; adjust the camera $\mathrm{f}$-stop to be $\mathrm{f} / 8$. This value is fixed through the whole calibration process. 
2. Adjust object distance and focus the lens such that the target image occupies $1 / 3$ to $1 / 2$ of imaging area of the CCD. This will reduce the uncertainty of image height measurement in pixels.

3. Take an image of the target and measure the distance from the lens to the target (i.e., the object distance) using a tape measure. Also measure the characteristic length of the target. This is shown in Figure A.2 below.

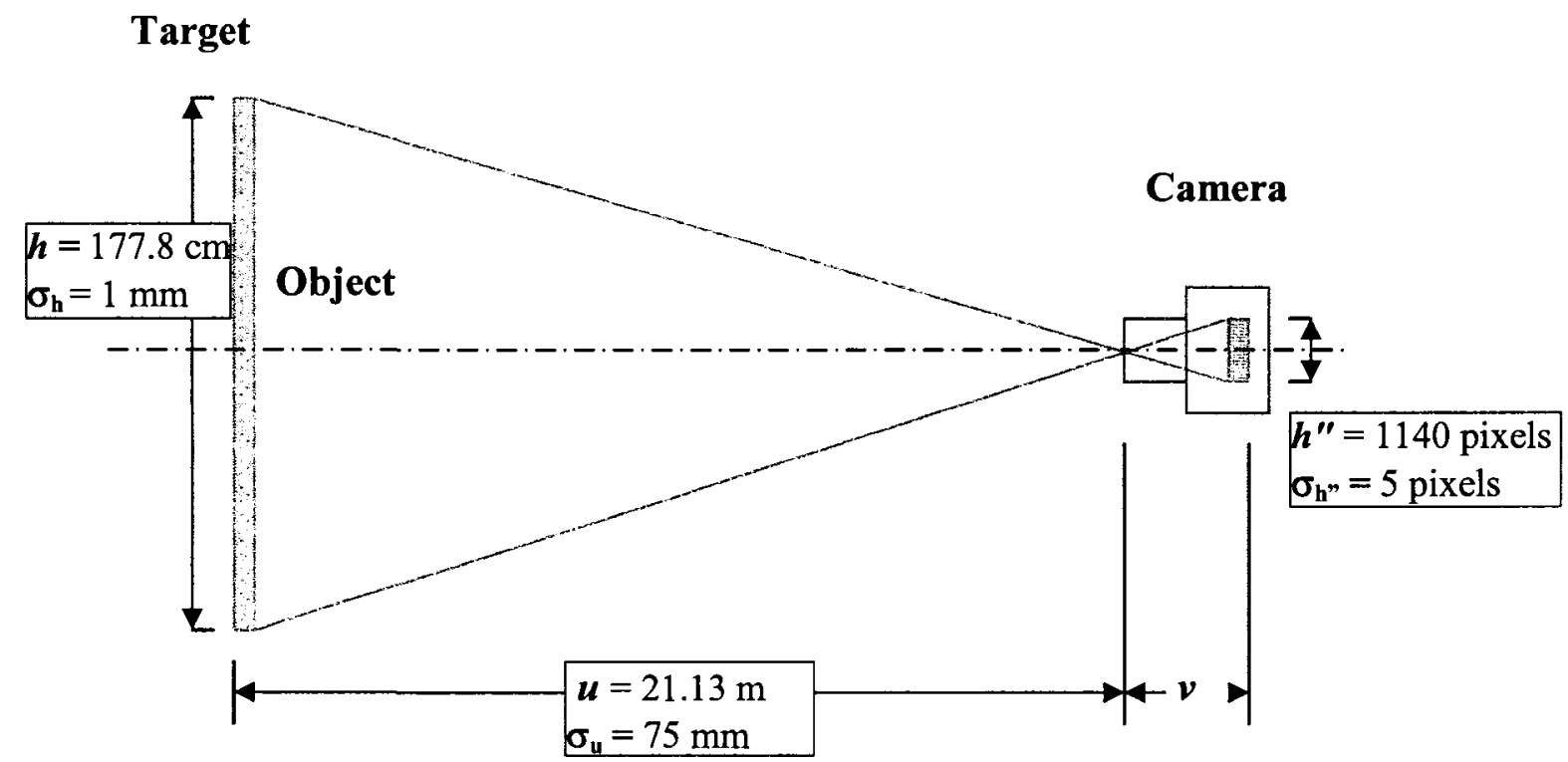

Figure A.2: Schematic of the position of the target and the camera.

4. Solve for magnification using the measured target height and the image height in the image, and using Equation A.5.

$$
M=\frac{h^{\prime \prime} \times \text { pixel size }}{h}=\frac{1140[\text { pixel }] \times 7.8[\mathrm{um} / \text { pixel }]}{177.8[\mathrm{~cm}]}=5.00 \times 10^{-3}
$$

5. Calculate the uncertainty of magnification, $\sigma_{\mathrm{M}} \cdot \sigma_{\mathrm{M}}$ is calculated following an error propagation analysis on Equation A.5. 


$$
\begin{aligned}
& \left(\frac{\sigma_{\mathrm{M}}}{M}\right)^{2}=\left(\frac{\sigma_{\mathrm{h}}}{h^{\prime \prime}}\right)^{2}+\left(\frac{(-1) \sigma_{\mathrm{h}}}{h}\right)^{2} \\
& \Rightarrow\left(\frac{\sigma_{\mathrm{M}}}{5.00 \times 10^{-3}}\right)^{2}=\left(\frac{5[\text { pixels }]}{1140[\text { pixels }]}\right)^{2}+\left(\frac{0.1[\mathrm{~cm}]}{177.8[\mathrm{~cm}]}\right)^{2} \\
& \Rightarrow \sigma_{\mathrm{M}}=2.211 \times 10^{-5}
\end{aligned}
$$

6. Calculate focal length of the lens, $f$, using Equation A.7.

$$
f=\frac{M \cdot u}{M+1}=\frac{5.00 \times 10^{-3} \cdot 21.13[\mathrm{~m}]}{5.00 \times 10^{-3}+1}=0.105[\mathrm{~m}]
$$

7. Calculate the uncertainty of focal length, $\sigma_{\mathrm{f}}$, using Equation A.10.

$$
\begin{aligned}
& \left(\frac{\sigma_{\mathrm{f}}}{f}\right)^{2}=\left(\frac{\sigma_{\mathrm{u}}}{u}\right)^{2}+\left(\frac{\sigma_{\mathrm{M}}}{M(M+1)}\right)^{2} \\
& \Rightarrow\left(\frac{\sigma_{\mathrm{f}}}{0.105[\mathrm{~m}]}\right)^{2}=\left(\frac{0.075[\mathrm{~m}]}{21.13[\mathrm{~m}]}\right)^{2}+\left(\frac{2.211 \times 10^{-5}}{5.00 \times 10^{-3}\left(5.00 \times 10^{-3}+1\right)}\right)^{2} \\
& \Rightarrow \sigma_{\mathrm{f}}=5.944 \times 10^{-4}[\mathrm{~m}]
\end{aligned}
$$

With the focal length and focal length uncertainty now quantified, it is possible to consider the uncertainty of the magnification in a field measurement.

8. Keep the same f-num in imaging a plume as in imaging the target. This is important since the effective focal length of the lens may vary with the f-num due to spherical aberrations. Adjust lenses to focus on a plume and take an image.

9. Measure the object distance from the plume to the camera using the laser range finder. The uncertainty of the object distance $\left(\sigma_{u}\right)$ depends on the accuracy of the laser range finder. For the current range finder, the accuracy is \pm 6 inches for the range from $2-600 \mathrm{~m}$.

10. Calculate magnification in the plume image using Equation A.8, assuming the plume is $100 \mathrm{~m}$ away from the camera (see Figure A.3). 


$$
M=\frac{f}{u-f}=\frac{0.105[\mathrm{~m}]}{100[\mathrm{~m}]-0.105[\mathrm{~m}]}=1.053 \times 10^{-3}
$$

Plume

Camera

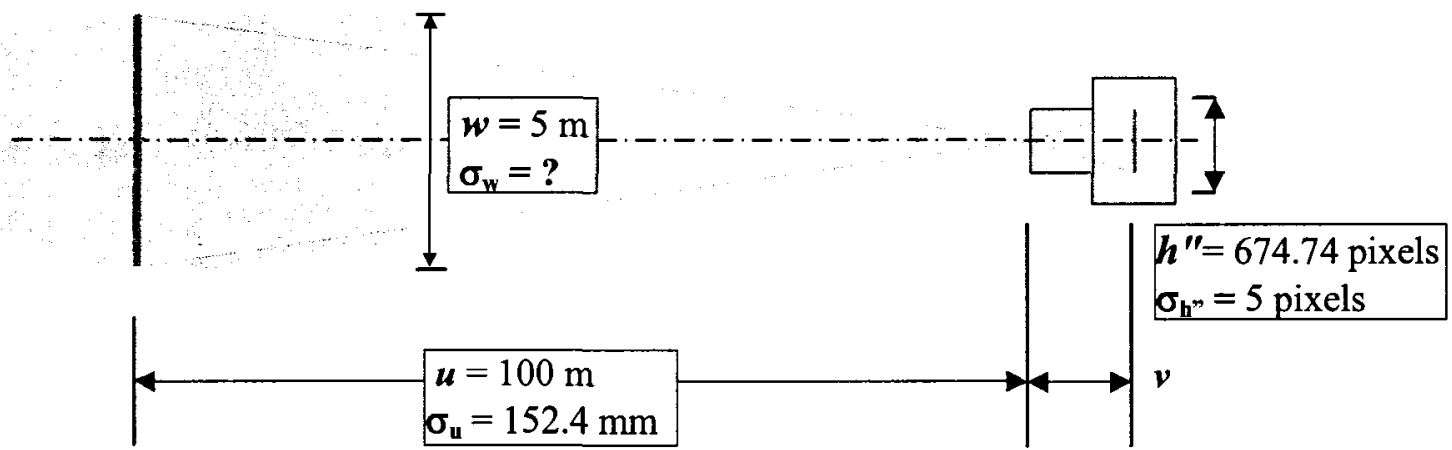

Figure A.3: Schematic of the position of a plume and the camera.

11. Calculate uncertainty in magnification by substituting the values from step 6 and 7 into Equation A.9.

$$
\begin{aligned}
& \left(\frac{\sigma_{\mathrm{M}}}{M}\right)^{2}=\left(\frac{\sigma_{\mathrm{u}}}{f-u}\right)^{2}+\left(\frac{\sigma_{\mathrm{f}} \cdot u}{f(u-f)}\right)^{2} \\
& \Rightarrow\left(\frac{\sigma_{\mathrm{M}}}{1.053 \times 10^{-3}}\right)^{2}=\left(\frac{152.4[\mathrm{~mm}]}{0.105[\mathrm{~m}]-100[\mathrm{~m}]}\right)^{2}+\left(\frac{5.944 \times 10^{-4}[\mathrm{~m}] \cdot 100[\mathrm{~m}]}{0.105[\mathrm{~m}](100[\mathrm{~m}]-0.105[\mathrm{~m}])}\right)^{2} \\
& \Rightarrow \sigma_{\mathrm{M}}=6.169 \times 10^{-6} \\
& \quad \frac{\sigma_{\mathrm{M}}}{M}=\frac{6.169 \times 10^{-6}}{1.053 \times 10^{-3}}=0.586 \%
\end{aligned}
$$

After gaining $\frac{\sigma_{\mathrm{M}}}{M}$, we can calculate relative uncertainty contribution to $\dot{m}_{\text {soot }}$ via

Equation A.4.

$$
\frac{\sigma_{\dot{m}_{\mathrm{soot}}}}{\dot{m}_{\mathrm{soot}}}=\sqrt{\left(\frac{\sigma_{\mathrm{M}}}{M}\right)^{2}}=\sqrt{\left(\frac{6.169 \times 10^{-6}}{1.053 \times 10^{-3}}\right)^{2}}=0.586 \%
$$


Therefore, the spatial calibration error in current configuration would contribute relative error in soot mass yield rate of only $0.586 \%$ if the plume is $100 \mathrm{~m}$ away. By doing the analysis for different object distance from $10 \mathrm{~m}$ to $600 \mathrm{~m}$, the results are shown in Figure A.4. The further away the plume is, the lower the relative error of magnification is. And thus lower the uncertainty contribution of the magnification to the total uncertainty of the soot mass yield rate. When the object distance is above $50 \mathrm{~m}$, the relative error becomes somewhat invariant with increasing distance as predicted from Equation A.9 where the first term goes to zero as $u$ becomes large relative to $f$ and the second term simplifies to $\sigma_{\mathrm{f}} / f$, the relative uncertainty of the focal length of the lens.

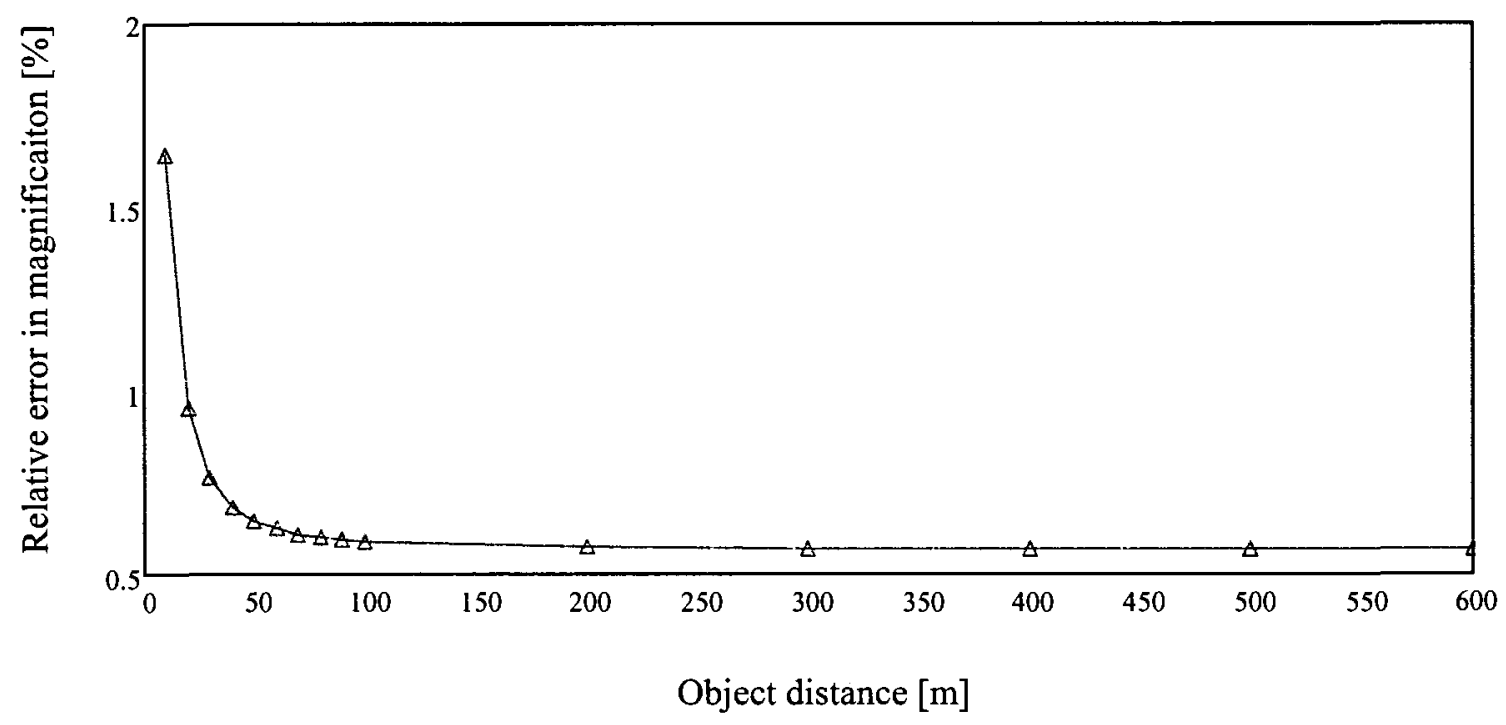

Figure A.4: Relative errors in magnification/ soot mass yield rate at different object distances from $10 \mathrm{~m}$ to $600 \mathrm{~m}$.

\section{A.2.1 Plume Width and Object Distance Effects}

This completes the magnification calibration for a field setting. The result can be carried one step further to determine the relative uncertainty in plume width measurement from plume images. It is useful to know the plume width to get some sense of plume sizes 
relative to the object distances. However, it is noted that the uncertainty of plume width is not a factor which will directly contribute to the uncertainty in $\dot{m}_{\text {soot }}$ (see Equation A.3).

12. Calculate the relative uncertainty $\left(\frac{\sigma_{\mathrm{w}}}{w}\right)$ in estimating the plume width using values obtained from previous steps and assuming the width of a plume $(w)$ is 5 $\mathrm{m}$.

$$
\begin{aligned}
& w=\frac{h^{\prime \prime}}{M} \\
& \Rightarrow h^{\prime \prime}=w \cdot M=\frac{5[\mathrm{~m}] \cdot 1.053 \times 10^{-3}}{7.8[\mathrm{um} / \mathrm{pixel}]}=674.7[\mathrm{pixel}]
\end{aligned}
$$

13. $\frac{\sigma_{\mathrm{w}}}{w}$ is calculated following an error propagation analysis on Equation A.11.

$$
\begin{aligned}
& \left(\frac{\sigma_{\mathrm{w}}}{w}\right)^{2}=\left(\frac{\sigma_{\mathrm{h}^{\prime \prime}}}{h^{\prime \prime}}\right)^{2}+\left(\frac{(-1) \sigma_{\mathrm{M}}}{M}\right)^{2} \\
& \Rightarrow\left(\frac{\sigma_{\mathrm{w}}}{w}\right)^{2}=\left(\frac{5[\text { pixel }]}{674.7[\text { pixel }]}\right)^{2}+\left(\frac{6.169 \times 10^{-6}}{1.053 \times 10^{-3}}\right)^{2} \\
& \Rightarrow \frac{\sigma_{\mathrm{w}}}{w}=0.95 \%
\end{aligned}
$$

The relative uncertainty in plume width $\left(\frac{\sigma_{\mathrm{w}}}{w}\right)$ varies with different plume sizes and different object distances. The follow results in Figure A.5 show the relative uncertainties estimated for plume widths of $1 \mathrm{~m}$ to $5 \mathrm{~m}$ and object distances from $10 \mathrm{~m}$ to $600 \mathrm{~m} .600 \mathrm{~m}$ is the longest distance which could be measured using the current laser range finder. 
At different object distance from $10 \mathrm{~m}$ to $100 \mathrm{~m}$

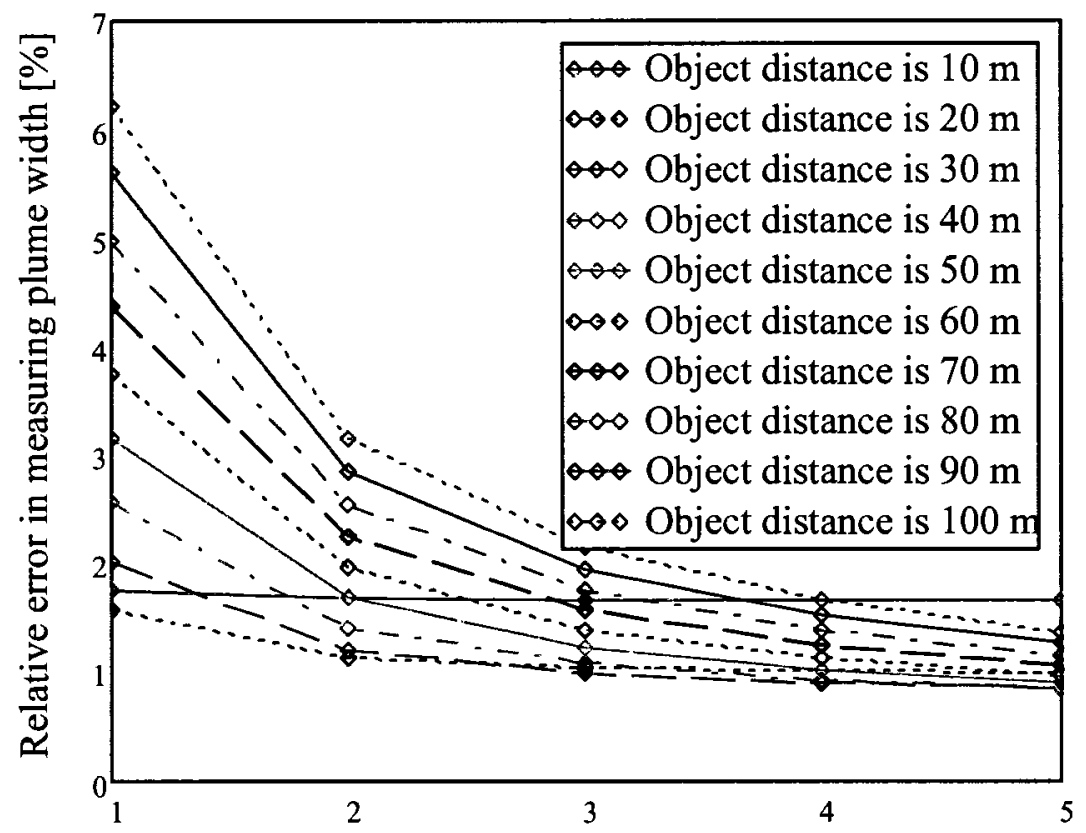

Plume width [m]

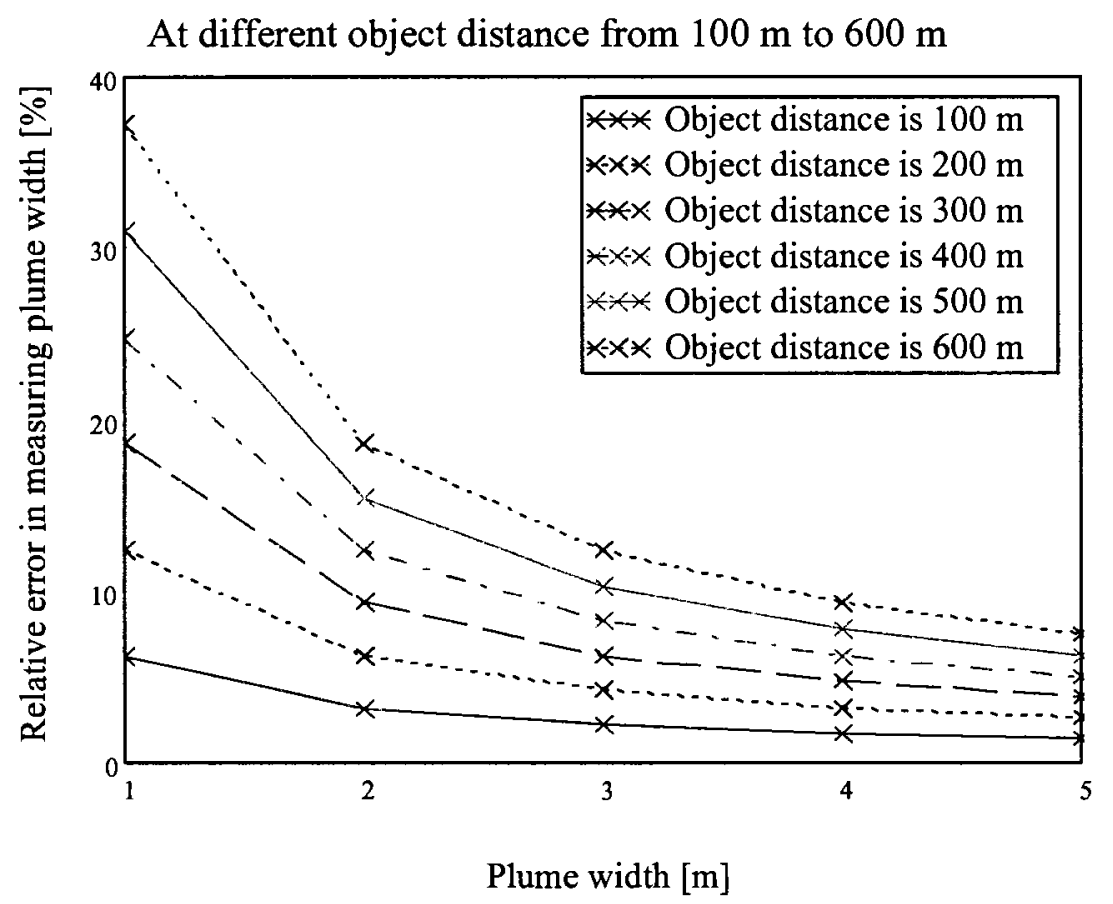

Figure A.5: Relative uncertainties in plume width for plume width from $1 \mathrm{~m}$ to $5 \mathrm{~m}$ at object distance a) from $10 \mathrm{~m}$ to $100 \mathrm{~m}$ b) from $100 \mathrm{~m}$ to $600 \mathrm{~m}$. 
Figure A.5 illustrates that the relative error $\left(\sigma_{w} / w\right)$ decreases with increasing plume width and decreasing object distance. The error is less than $1.4 \%$ for object distance between $20 \mathrm{~m}$ to $100 \mathrm{~m}$ (Figure A.5a) and less than $7.4 \%$ for object distance between 200 $\mathrm{m}$ to $600 \mathrm{~m}$ (Figure A.5b) if the plume size is $5 \mathrm{~m}$ wide. If the object distance is less than $10 \mathrm{~m}$, the relative error is insensitive with the plume size (see the red line in Figure A.5a). This is because the uncertainty from the range finder becomes more dominant than the uncertainty from magnification when object distance is less than $10 \mathrm{~m}$. For a plume width of $1 \mathrm{~m}$, the camera should be positioned less than $100 \mathrm{~m}$ in order to achieve the relative uncertainty of $6.2 \%$ or less (See Figure A.5a).

\section{A.2.2 Nearest Limit of Object Distance and Associated Error}

From previous background interpolation analysis, we know the plume width relative to the image width (i.e., the plume size) should be less than $30 \%$ or $20 \%$ in order to achieve minimum uncertainty in soot emission rate due to background interpolation algorithm. Based on $30 \%$ of plume size, an estimation of object distance, relative error in magnification, and associated error in soot yield rate is demonstrated below.

For PIXIS 1024BR scientific camera, which will be used in the field measurement, the image size is $13.312 \mathrm{~mm} \times 13.312 \mathrm{~mm}$. $30 \%$ plume size corresponds to plume width of $3.994 \mathrm{~mm}$ in the image (i.e., $w_{\text {image }}$ ). The magnification can be expressed by

$$
M=\frac{w_{\text {image }}}{w},
$$

Combined above equation with Equation A.8, we can solve for the object distance $u$, assuming the plume width of $1 \mathrm{~m}$ 


$$
u=\frac{f \cdot w}{w_{\text {image }}}+f=\frac{0.105[\mathrm{~m}] \cdot 1[\mathrm{~m}]}{3.994 \times 10^{-3}[\mathrm{~m}]}+0.105[\mathrm{~m}]=26.4[\mathrm{~m}]
$$

For a $1 \mathrm{~m}$ wide plume, to keep the plume size less than $30 \%$, the distance between the camera and the plume should be no less than $26.4 \mathrm{~m}$. Using Equation A.9 again, the relative error in magnification can be calculated as follow.

$$
\begin{aligned}
& \left(\frac{\sigma_{\mathrm{M}}}{M}\right)=\sqrt{\left(\frac{\sigma_{\mathrm{u}}}{f-u}\right)^{2}+\left(\frac{\sigma_{\mathrm{f}} \cdot u}{f(u-f)}\right)^{2}} \\
& \Rightarrow=\sqrt{\left(\frac{152.4[\mathrm{~mm}]}{0.105[\mathrm{~m}]-26.4[\mathrm{~m}]}\right)^{2}+\left(\frac{5.944 \times 10^{-4}[\mathrm{~m}] \cdot 26.4[\mathrm{~m}]}{0.105[\mathrm{~m}](26.4[\mathrm{~m}]-0.105[\mathrm{~m}])}\right)^{2}} \\
& \Rightarrow 0.81 \%
\end{aligned}
$$

Once again, the associated relative uncertainty in $\dot{m}_{\text {soot }}$ contributed by magnification error is obtained via Equation A.4.

$$
\frac{\sigma_{\dot{m}_{\mathrm{soot}}}}{\dot{m}_{\mathrm{soot}}}=\sqrt{\left(\frac{\sigma_{\mathrm{M}}}{M}\right)^{2}}=0.81 \%
$$

Therefore, the spatial calibration error in current optical system would contribute relative error in soot yield rate of only $0.81 \%$ if the plume width of $1 \mathrm{~m}$ and the plume occupies the image less than $30 \%$.

Repeat the above analysis for different plume widths but keeping the plume sizes in the images less than $30 \%$, the object distance and the relative uncertainty in mass flow rate of soot are shown in Figures A.6. Figure A.6 shows that the object distance increases linearly with increasing the plume width and the associated error in soot yield rates decreases with increasing the plume width but keep the plume size less than a certain percentage of the image. For $1 \mathrm{~m}$ wide plume, the camera should be positioned no less than $26.4 \mathrm{~m}$ away from the plume in order to keep the plume width less than $30 \%$ 
of the image. As shown in Figure A.7, the spatial calibration error in $\dot{m}_{\text {soot }}$ is $0.81 \%$ by current spatial calibration system when the object distance of $26.4 \mathrm{~m}$ for a $1 \mathrm{~m}$ wide plume.

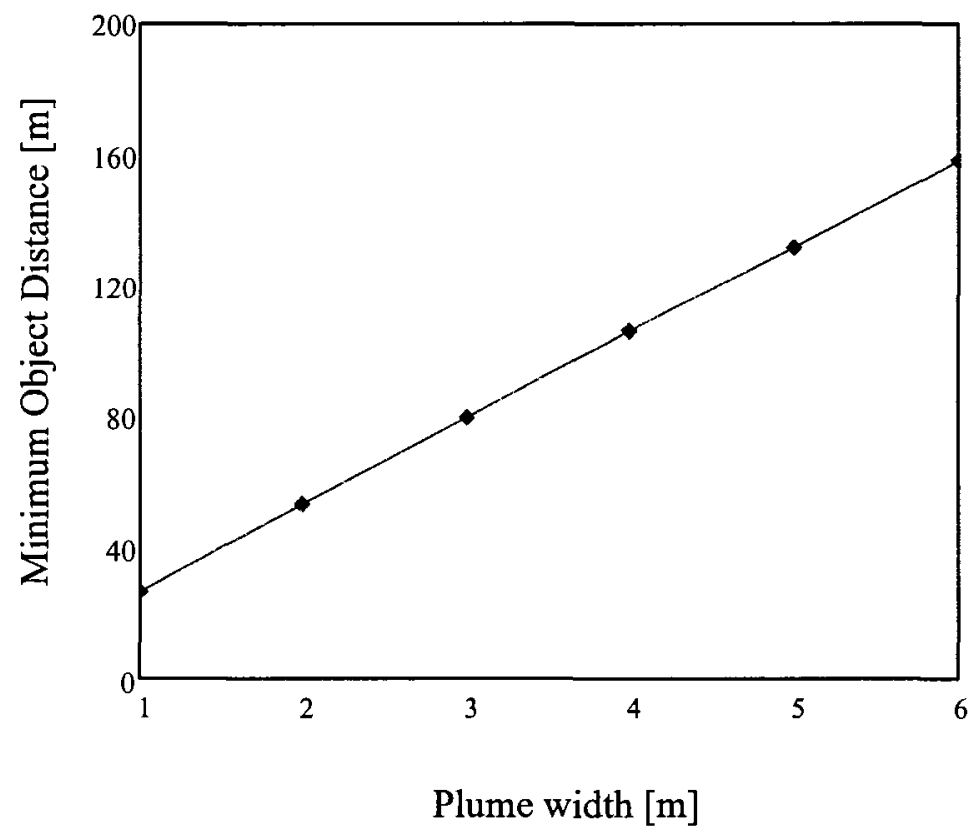

Figure A.6: Recommended distances of the observer from a plume for $30 \%$ plume size. 


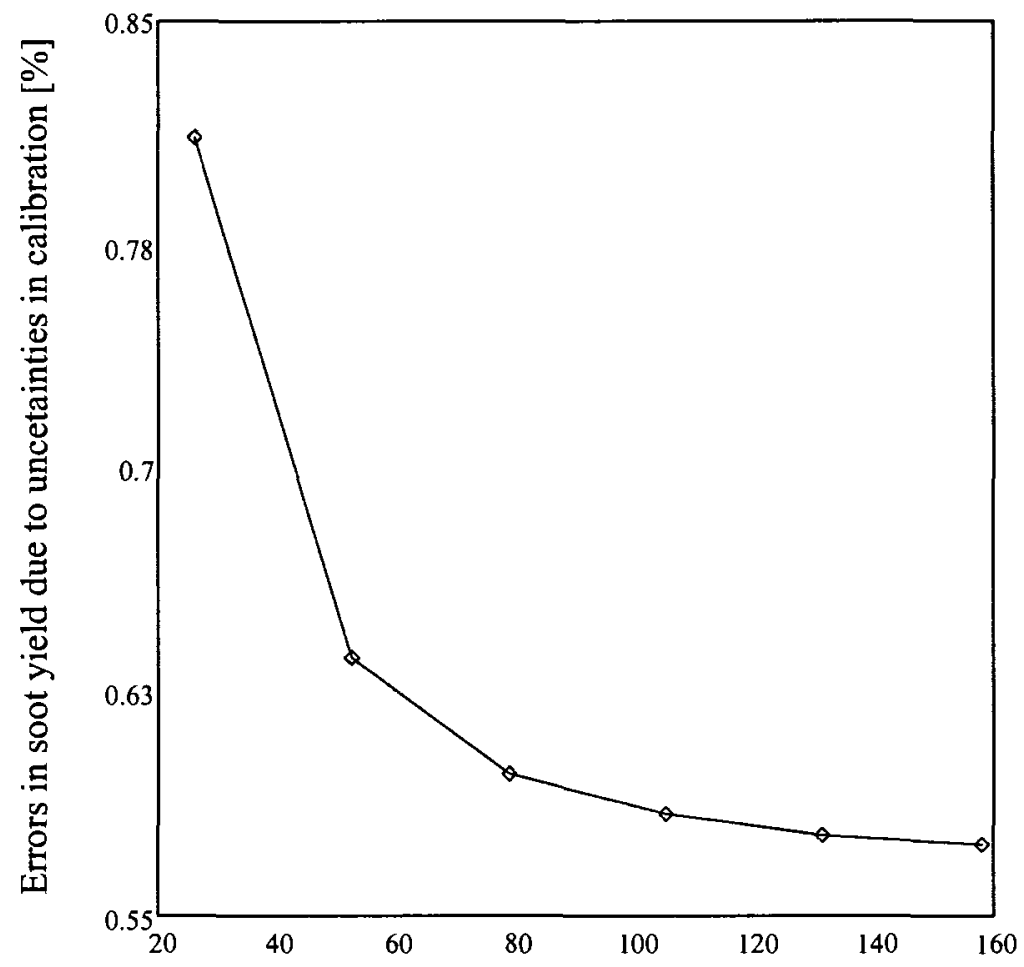

Object distance $[\mathrm{m}]$

Figure A.7: Associated errors in soot yield rates due to uncertainties in calibration for $30 \%$ plume size.

For the plume size of $20 \%$, simulation results of minimum object distances and associated errors in soot emission rates are shown in Figures 4.7 and 4.8 . For $1 \mathrm{~m}$ wide plume, the minimum object distance should be no less than $39.5 \mathrm{~m}$ away from the plume in order to keep the plume width less than $20 \%$ of the image. As shown in Figure 4.8 , the spatial calibration error in current optical system would contribute relative error in soot yield rate of $0.68 \%$ when the object distance of $39.5 \mathrm{~m}$ for the plume width of $1 \mathrm{~m}$ and the plume occupies the image less than $20 \%$. By selecting a longer focal length $(f)$ lens, we can increase the object distance for the same size of plume and thus decrease the magnification error therefore the uncertainty of mass flow rate of soot. 


\section{APPENDIX B: Beam Steering}

In an optical system, beam steering occurs when the refractive index of a medium varies perpendicularly to the path along which the light beam is transmitted. Gradients in temperature and mixture composition in a plume can cause gradients in the refractive index across the plume. Such refractive index gradients deflect the beam as it passes through the plume and have the potential to introduce a further uncertainty into the measurement. A plume is assumed to be composed of dry air because the plume is principally air due to high dilution ratio as mentioned in Chapter 2. The major cause of beam steering is due to the temperature gradient, $\frac{\partial T}{\partial y}$. Beam steering in terms of deflection angle, $\theta$, is proportional to

$$
\theta \propto\left(\frac{1}{T^{2}}\right)\left(\frac{\partial T}{\partial y}\right)
$$

where $T$ is the temperature of the plume and $y$ is the axis perpendicular to the optical axis. Therefore, beam steering reaches its highest value when temperature is low and temperature gradient is high. This is most likely to occur near the edges of the plume.

The beam steering phenomenon is shown schematically in Figure B.1. A light beam starts at an offset position of $y_{o}$ entering the plume. The beam is bent away from the optical axis as it passes through the plume with a final deflection angle, $\theta_{\text {ster, }}$, as it leaves the plume. The difference between the true beam path through the plume and the 
unsteered beam path (i.e., $\left.y_{0}\right)$ is defined as the deflection displacement, $y_{\text {steer }}(x)$ and is important since it defines an uncertainty of the measured path through the plume. Because of beam steering, there will also be a distortion in the image of the beam on the detector. This distortion can be quantified by projecting the final trajectory of the beam after it leaves the plume back to the object plane at the centre of the plume. The intersection is denoted $b$ and the difference between $y_{o}$ and $b$ is the distortion that would be observed in the plume image (once multiplied by the magnification of the imaging optics). The distortion relative to the object is denoted $y_{\text {distortion }}$.

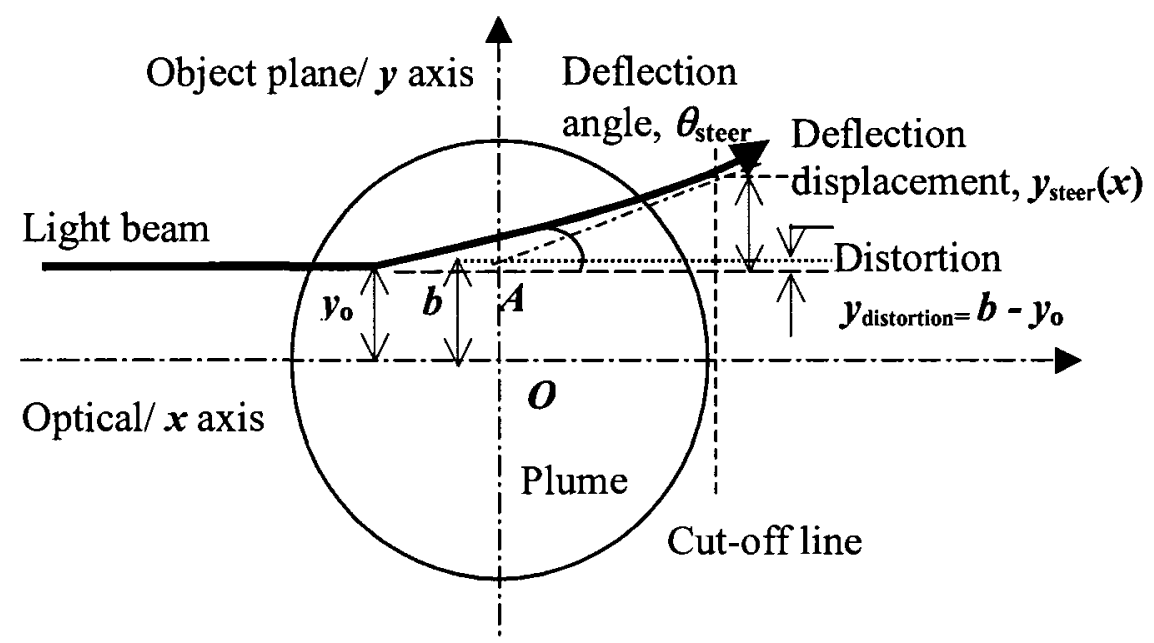

Figure B.1: Schematic beam steering through a circular plume.

To quantify the beam steering uncertainty in plume transmissivity measurements, a MathCAD computer model developed by NRC combustion group is used [Thomson, 2004]. The model requires an input temperature field. Poudenx [2000] measured and established mean temperature maps for 149 cross-sections of plumes of diffusion flame within a controlled wind tunnel. The study showed that in low crosswind conditions, the plume peak temperature is on the order of $357^{\circ} \mathrm{C}$ and plumes appear kidney shaped. At 
higher crosswinds the plume cross-sections were circular. As a first analysis, a $1 \mathrm{~m}$ diameter plume is simulated as having a Gaussian axi-symmetric temperature distribution with centreline temperature of $357^{\circ} \mathrm{C}$ dropping to an ambient temperature of $0^{\circ} \mathrm{C}$ with the plume radius corresponding to $3 \sigma$.

The simulation was applied to a region from $-0.9 \mathrm{~m}$ to $0.9 \mathrm{~m}$ in the $\mathrm{x}$ direction for $y_{o}$ values ranging from 0 to $0.95 \mathrm{~m}$. The simulated steering, $y_{\text {steer }}(x)$, is shown in Figure B.2a. Because beam deflection which occurs after the beam leaves the plume is not important to the characterization of the transmissivity through the plume, the radial position of $0.5 \mathrm{~m}$ is chosen as the cut off limit in the Figure B.2a. 

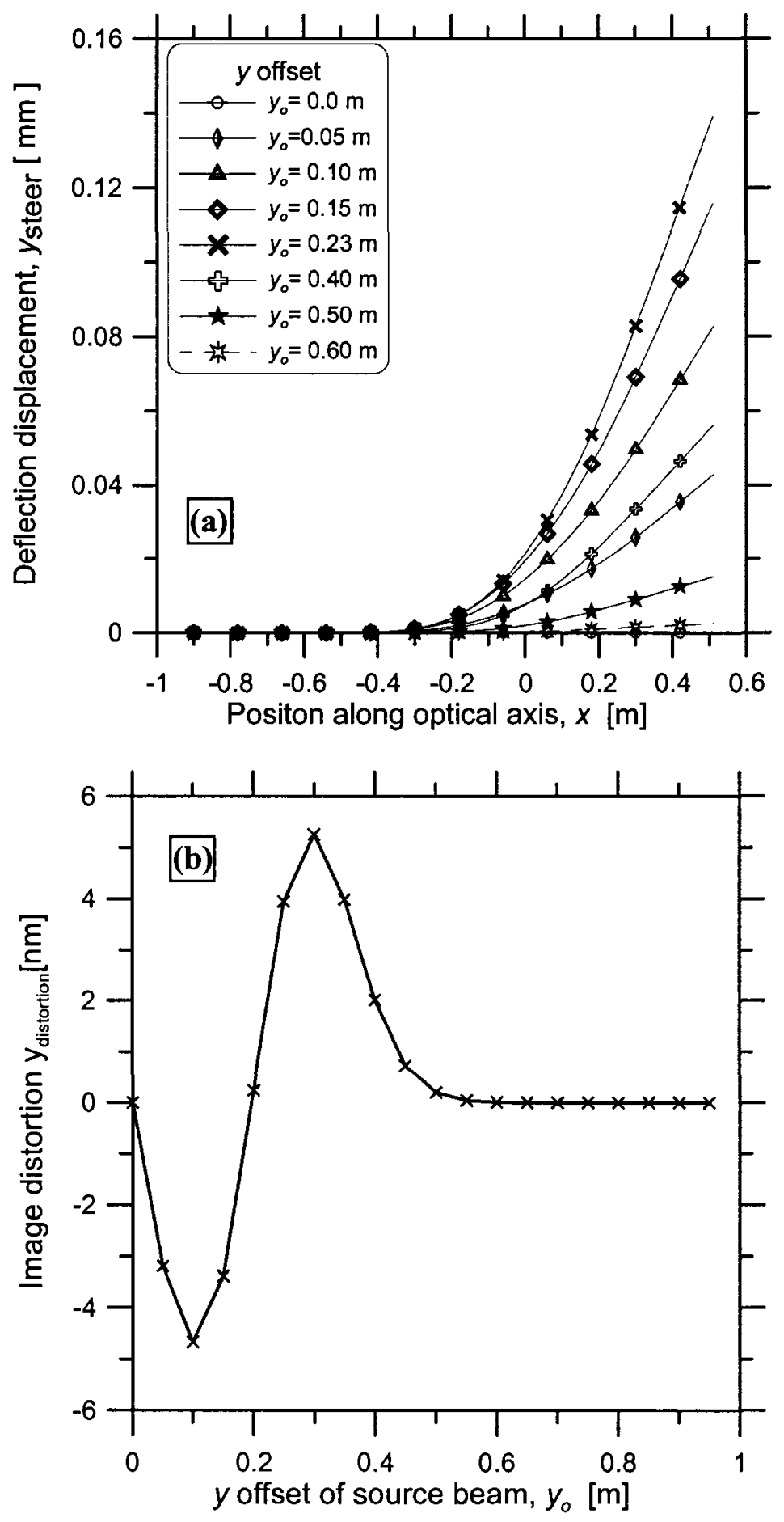

Figure B.2: a) Beam steering $y_{\text {steer }(x)}$ simulation, $1 \mathrm{~m}$ diameter plume, Gaussian temperature profile. b) Corresponding image distortion. 
The light beams start steering at $-0.3 \mathrm{~m}$ within the plume. The maximum deflection displacement occurs for $y_{0}=0.23 \mathrm{~m}$ and is less than $0.16 \mathrm{~mm}$ and maximum deflection angle $\left(\theta_{\text {steer}}\right)$ is less than $0.3 \mathrm{mrad}$ (not shown). The beam distortion (in $\mathrm{nm}$ ) in the image is estimated and shown in Figure B.2b. With increasing $y_{\mathrm{o}}$, beams are distorted towards the optical axis. After passing a certain value of $y_{0}$, the beam starts being distorted away from the optical axis. The certain value depends on the temperature profile within the plume. When $y_{0}$ moves outside of the plume, the beam distortion becomes zero due to temperature gradient tends to be zero. For the assumed conditions, the calculated maximum distortion is $2.58 \mathrm{~nm}$ and is clearly negligible. 